Portland State University

PDXScholar

7-14-2020

\title{
Detecting Reinforcement Patterns in the Stream of Naturalistic Observations of Social Interactions
}

James Lamar DeLaney 3rd

Portland State University

Follow this and additional works at: https://pdxscholar.library.pdx.edu/open_access_etds

Part of the Developmental Psychology Commons

Let us know how access to this document benefits you.

\section{Recommended Citation}

DeLaney 3rd, James Lamar, "Detecting Reinforcement Patterns in the Stream of Naturalistic Observations of Social Interactions" (2020). Dissertations and Theses. Paper 5553.

https://doi.org/10.15760/etd.7427

This Thesis is brought to you for free and open access. It has been accepted for inclusion in Dissertations and Theses by an authorized administrator of PDXScholar. Please contact us if we can make this document more accessible: pdxscholar@pdx.edu. 
Detecting Reinforcement Patterns in the Stream of Naturalistic Observations of Social Interactions

\author{
by \\ James Lamar DeLaney $3^{\text {rd }}$
}

A thesis submitted in partial fulfillment of the requirements for the degree of

\author{
Master of Science \\ in \\ Psychology
}

Thesis Committee:

Thomas Kindermann, Chair

Jason Newsom

Ellen A. Skinner

Portland State University 


\begin{abstract}
How do consequences affect future behaviors in real-world social interactions? The term positive reinforcer refers to those consequences that are associated with an increase in probability of an antecedent behavior (Skinner, 1938). To explore whether reinforcement occurs under naturally occuring conditions, many studies use sequential analysis methods to detect contingency patterns (see Quera \& Bakeman, 1998). This study argues that these methods do not look at behavior change following putative reinforcers, and thus, are not sufficient for declaring reinforcement effects arising in naturally occuring interactions, according to the Skinner's (1938) operational definition of reinforcers.

This study presents the conceptual and technical development of an algorithmic strategy and web-app used to restructuring naturalistic observations in a way that can be used to assess behavior change as a function of putative reinforcers. I demonstrate this strategy by re-examining some of the hypotheses Sage and Kindermann's (1999) study on contingency patterns across fifth and sixth grade students.

Using methods of generalized estimating equations (e.g. Liang \& Zeger, 1986) and Allison and Liker (1982) z-scores, this study found full support for reinforcement effects of Social Approval for children's Off-Task classroom behaviors, and partial support for Social Approval following On-Task behaviors. Social Disapproval was shown to reinforce Off-Task behaviors, contrary to expectations. This paper concludes by a comprehensive discussion of these results and directions for future modifications of these reinforcement detection strategies.
\end{abstract}




\section{Acknowledgements}

I want to thank my committee members for their guidance. Specifically, I want to thank Dr. Jason Newsom for his visions in guiding the primary analysis and extending the scope of the study beyond its original capacity. I want to thank Dr. Ellen Skinner for being the 'glue' behind multiple perspectives behind the study and for her strong patience throughout the process. Finally, I want to thank Dr. Thomas Kindermann for providing the vision behind this project and expanding my vision for my research career. Dr. Kindermann's passion and support has been a true inspiration and will continue to shape my perspectives, attitudes, and forward thinking, with regards to the synthesis of the intellectual and humane in my future endeavors. All three of my committee members have embodied patience, and for this, I have the uttermost gratitiude.

I want to thank my family for embodying love, perseverance, humility, and compassion (among all other good things), from day one till the future. My understanding of being a good human being comes directly from their examples. I want to thank all my friends, family, and lab-mates for continuing to inspire and teach me new lessons on being the best we can be. 


\section{Table of Contents}

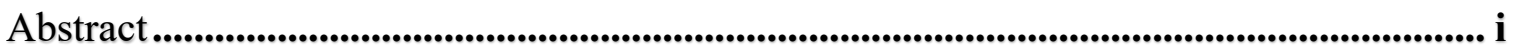

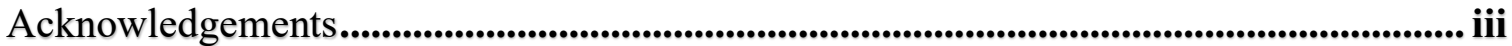

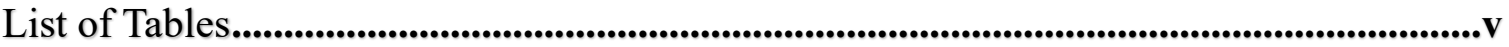

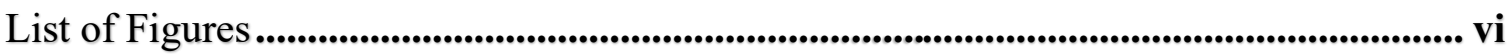

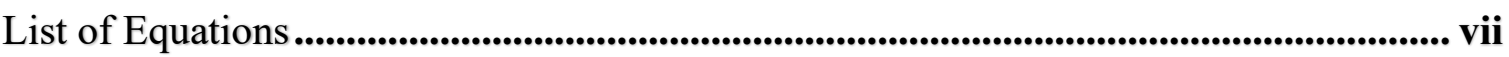

Chapter 1

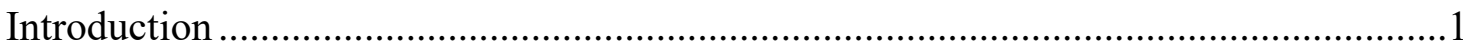

Chapter 2

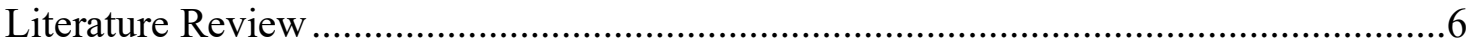

Chapter 3

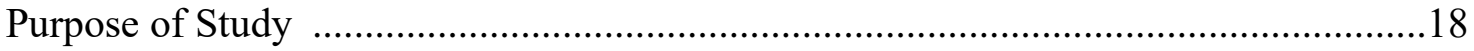

Chapter 4

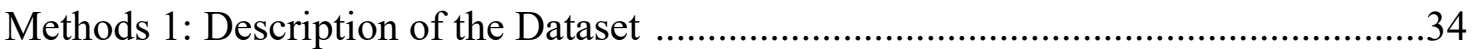

Chapter 5

Methods 2: Describing Reinforcement in the Stream of Observations ........................41

Chapter 6

Methods 3: An Online Application for Detecting Reinforcers ...................................74

Chapter 7

Results

Chapter 8

Discussion

Appendix A. Experimental Demonstrations of Social Reinforcers in Classrooms

Appendix B. Non-Experimental Reinforcement Detection Strategies

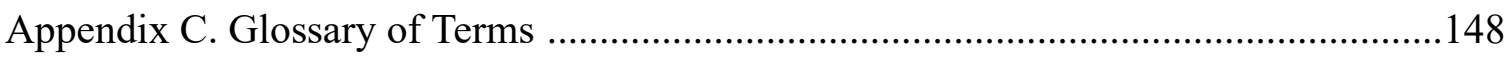

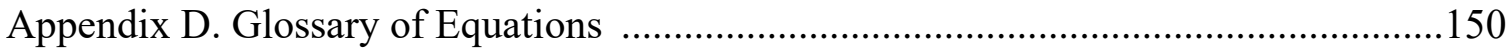

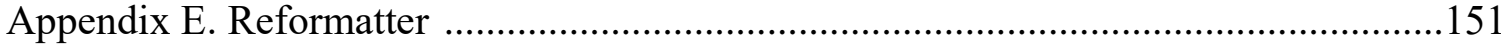

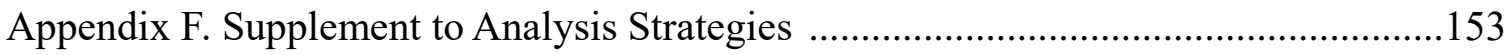

Appendix G. Detailed Review of Strategies ............................................................157 
Appendix H. Primary Analyses with Exchangeable Working Correlations ....................162 


\section{List of Tables}

Table 1. All lag-one conditional frequencies from Figure 1 .......................................... 24

Table 2. Focal children behavior codes .......................................................................... 38

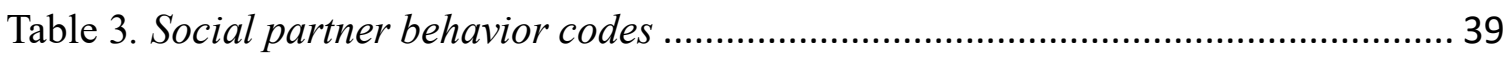

Table 4. Example long-formatted observational data file ............................................ 41

Table 5. Shortened example recounted data series (data-frame) ................................... 51

Table 6. Contingency table for recounted frequencies.................................................. 71

Table 7. Contingency table for average recounted frequencies ..................................... 73

Table 8. Contingency table for recomputed frequencies .............................................. 74

Table 9. Contingency table for recounted frequencies (table 6 reprint) ......................... 100

Table 10. Contingency table for average frequencies (table 7 reprint) .......................... 101

Table 11. Contingency table for recomputed frequencies (table 8 reprint)) ................... 102

Table 12. Proportions used in Allison and Liker (1982) z-scores .................................. 103

Table 13. Distribution of overall behaviors in original data-file ................................. 104

Table 14. Distribution of recounted behaviors for social approval as a reinforcer ....... 105

Table 15. Research question 1 hypothesis 1 primary analysis..................................... 106

Table 16. Research question 1 hypothesis 1 proportions ............................................ 107

Table 17. Research question 1 hypothesis 1 summary secondary analysis ................... 107

Table 18. Research question 1 hypothesis 2 primary analysis.................................... 109

Table 19. Research question 1 hypothesis 2 proportions .............................................. 109

Table 20. Research question 1 hypothesis 2 summary secondary analysis ................... 110

Table 21. Distribution of recounted behaviors for direct disapproval as a punisher ..... 111

Table 22. Research question 2 hypothesis 3 primary analysis..................................... 112

Table 24. Research question 2 hypothesis 3 summary secondary analysis .................... 113

Table 25. Research question 2 hypothesis 3 proportions ............................................ 114

Table 26. Structure of the recounted observations for primary analysis ....................... 160

Table 27. Contingency table for the recounted frequencies using overall strategy ........ 160

Table 29. Recounted series contingency table for two focal children.............................. 162

Table 30. Lag-one conditional frequencies and proportions for red circles ................... 164

Table 31. Proportions used for the Allison and Liker (1982) z-score ........................... 165

Table 32. Flipped lag-one prediction $3 \times 3$ proportions ............................................ 166

Table 33. Proportions used for the Allison and Liker (1982) z-score on Green Circles. 166

Table 34. Average lag probability for green given red circles ..................................... 166

Table 35. Averaged flipped prediction proportions (Green $\mid$ Red) ................................. 167

Table 36. Research question 1 hypothesis 1 primary analysis....................................... 169

Table 37. Research question 1 hypothesis 2 primary analysis..................................... 169

Table 38. Research question 2 hypothesis 3 primary analysis................................... 170

Table 39. Comparison of GEE and logistic regression models ................................... 170 


\section{List of Figures}

Figure 1. General reinforcement detection measure (a.k.a. recounting procedure) ........... 3 Figure 2. Reinforcement detection measure (a.k.a. recounting procedure) (Figure 1

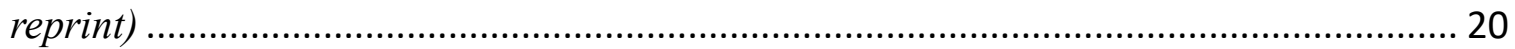

Figure 3. Conceptual depiction of contingency detection via lag-sequential analysis .... 23

Figure 4. Overall reinforcement detection measure (Figure 1 reprint) ........................... 34

Figure 5. Example classroom observation series.............................................................. 45

Figure 6. Recounting procedure applied to classroom observations to detect reinforcers.

Figure 7. Stepwise depiction of the recounting procedure ............................................ 49

Figure 8. Shortened example data series .......................................................................... 51

Figure 9. Illustrative example of overall matched pairs ................................................... 54

Figure 10. Data structure and transition table for matched pairs .................................. 54

Figure 11. Contingency table for marginal distribution of matched pairs. ...................... 56

Figure 12. Data structure and transition table for matched pairs .................................. 57

Figure 13. Contingency table for marginal distribution of matched pairs. ...................... 58

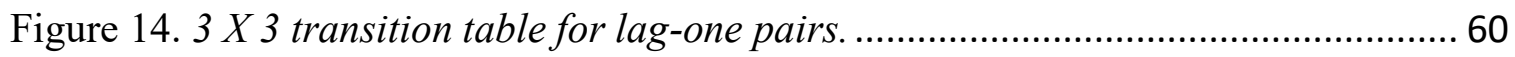

Figure 15. 2 X 2 marginal table for directed lag-one hypothesis. .................................. 61

Figure 16. Equivalency of marginal table of incomplete pairs and recounting procedure

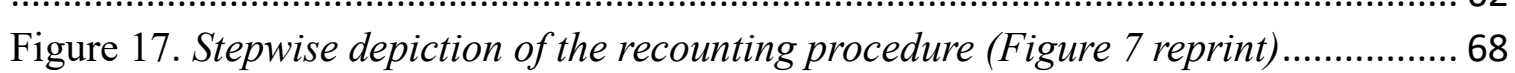

Figure 18. Reinforcement detection measure (a.k.a. recounting procedure) (Figure 1

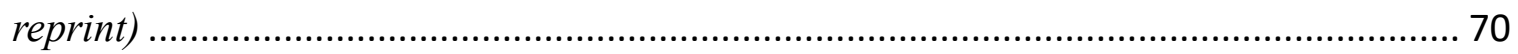

Figure 19. Reinforcinator Graphical User Interface .................................................. 77

Figure 20. Reinforcinator text panel ........................................................................ 79

Figure 21. Example of an appropriately reformatted dataset ...................................... 80

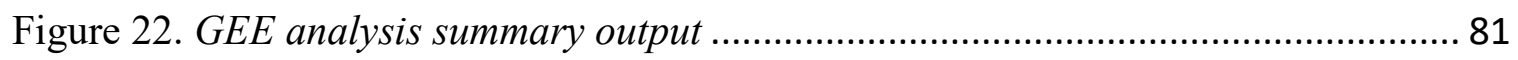

Figure 23. Recounted frequency table output .............................................................. 82

Figure 24. Reinforcement detection measure (a.k.a. recounting procedure) (Figure 1

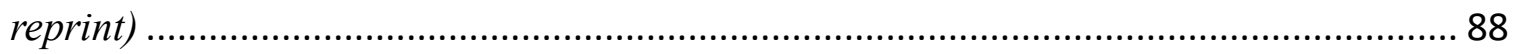

Figure 25. Stepwise depiction of the recounting procedure (Figure 7 reprint)................ 90

Figure 26. $3 \times 3$ transition table for lag-one pairs (Figure 14 reprint) ........................... 94

Figure 27. 2 X 2 marginal table for directed lag-one hypothesis (Figure 15 reprint) ...... 95

Figure 28. Equivalency of marginal table of incomplete pairs and recounting procedure

Figure 29. Reinforcement detection measure (a.k.a. recounting procedure) (Figure 1 reprint) 100 
Figure 30. Individual reinforcement patterns. .......................................................... 123

Figure 31. Example of an appropriately reformatted dataset ....................................... 158

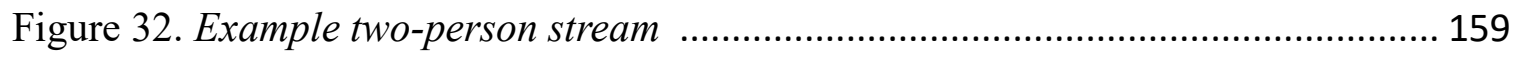

Figure 33. Matched pairs example algorithm ........................................................... 161

Figure 34. Overall application of recounting algorithm............................................ 162

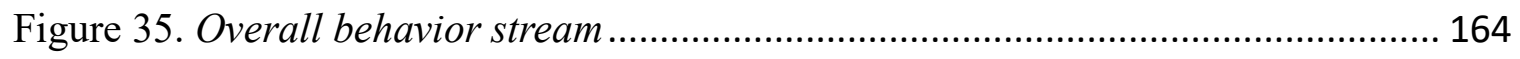

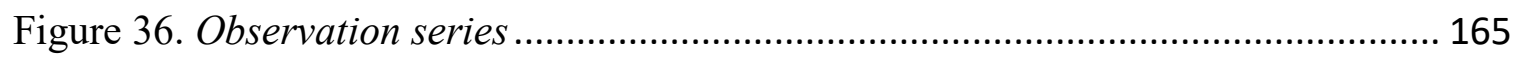

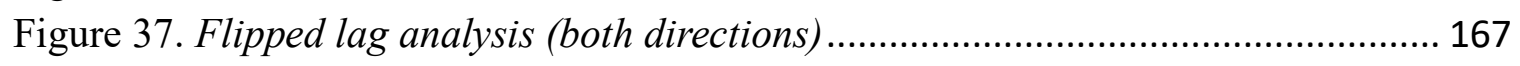




\section{List of Equations}

Equation 1. Allison and Liker (1982) binomial z-score for lag analysis ........................ 25

Equation 2. Mcnemar test statistic .......................................................................... 56

Equation 3. Allison and Liker z-score for recounted frequencies ................................... 72

Equation 4. Allison and Liker z-score for the average recounted frequencies ................ 74

Equation 5. Allison and Liker z-score for the recomputed frequencies ............................ 75

Equation 6. Binomial $z$ test for lagged dependence ...................................................... 83

Equation 7. Allison and Liker Z-score (notation adjusted for contingency analysis)....... 95

Equation 8. Allison and Liker z-score adapted to reinforcer analysis (re-print) ............ 103

Equation 12. Equation for construction of matched pairs ............................................. 161

Equation 13. Binomial $Z$ test for lagged dependence .................................................. 165 


\section{Chapter 1}

\section{Introduction}

A behavioristic view of development focuses on the ways in which environments can provide conditions for individuals to grow behaviorally and to maintain growth over time (Schlinger, 2013). One set of corresponding mechanisms for this change are the immediate changes a behavior makes to its environments by eliciting specific consequences. It is the environmental consequences of behavior which can alter the probability of a behavior recurring in the future. As defined by B.F. Skinner (1953), "...any consequence of behavior which is rewarding, or more technically, reinforcing, increases the probability of further responding (B. F. Skinner, 1953; p. 345)".

With a rich history in developmental and social psychology (see Gewirtz \& Peláez-Nogueras, 1992; Maccoby, 2007 for historical reviews), the concept of social reinforcement is especially appealing because it defines an observable mechanism of social influence. Reinforcing events occur naturally during "the social exchange of some duration between two individuals "(Rubin, Bukowski, \& Parker, 1998, p. 12). Because of this theoretical and practical appeal, numerous observational studies have examined social interactions to identify potential social reinforcers involved in socialdevelopmental processes. Widely recognized examples are friendship making (Putallez \& Gottman, 1981; Snyder, Horsch, \& Childs, 1997) and compliant and coercive familial interactions (Dishion et al., 1994; Strand, Wahler, \& Herring, 2000). Additionally, observations have identified social consequences associated with more distal behavioral outcomes, such as future legal violations (Dishion, Spracklen, Andrews, \& Patterson, 
1996) and future academic achievement (Greenwood, Delquadri, and Hall, 1989). A common practice in these naturalistic observations is to assume that a consequence is reinforcing when it is positive in nature and when it follows the antecedent behavior immediately. The current thesis builds on the idea that, to date, no such naturalistic observational study has examined whether putatively reinforcing consequences actually reinforce the behaviors they follow by changing their future probability of occurring ${ }^{1}$.

Traditionally, it is common to test the claim that an event is a reinforcer by demonstrating its direct role in a process of learning, or ongoing behavior change. Behavioral experiments test this by determining if a behavior is function of its consequences. This is accomplished by manipulating pre-defined behavior-consequence relations (i.e. 'schedule of reinforcement') and then comparing subsequent behavior rates across these reinforcement schedules. If behavior is sensitive to changes in these conditions, then the consequence has demonstrated the function of a reinforcer (Skinner, 1956). But this is all investigated through experimental manipulation. To my knowledge, there are no existing methods used for detecting the function of consequences on future behaviors in naturalistic streams of observations.

The purpose of the current thesis is to design a measurement and testing strategy for the identification of reinforcers in any stream of naturalistic observations - based only on the observed effects a consequence has on the flow of behavior probability (see Figure 1 for an illustration). The idea is to use Skinner's definitional criterion that a reinforcer is an event that causes change in the probabilities of the behavior it follows. I have designed

\footnotetext{
${ }^{1}$ Skinner was skeptical of the term probability for its statistical implications (See Johnson \& Morris, 1987 for a comprehensive analysis of Skinner's use of the term). Accordingly, I use the term proportion in reference to the quantities being measured and compared in this thesis.
} 
such a measure through a recounting algorithm that compares behavior probabilities before and after all observations of potentially reinforcing events in natural streams of behavior. Figure 1 shows, as an example, three putatively reinforcing events (red circles) in an individual's natural stream of observed behavior.

Figure 1. General reinforcement detection measure (a.k.a. recounting procedure)

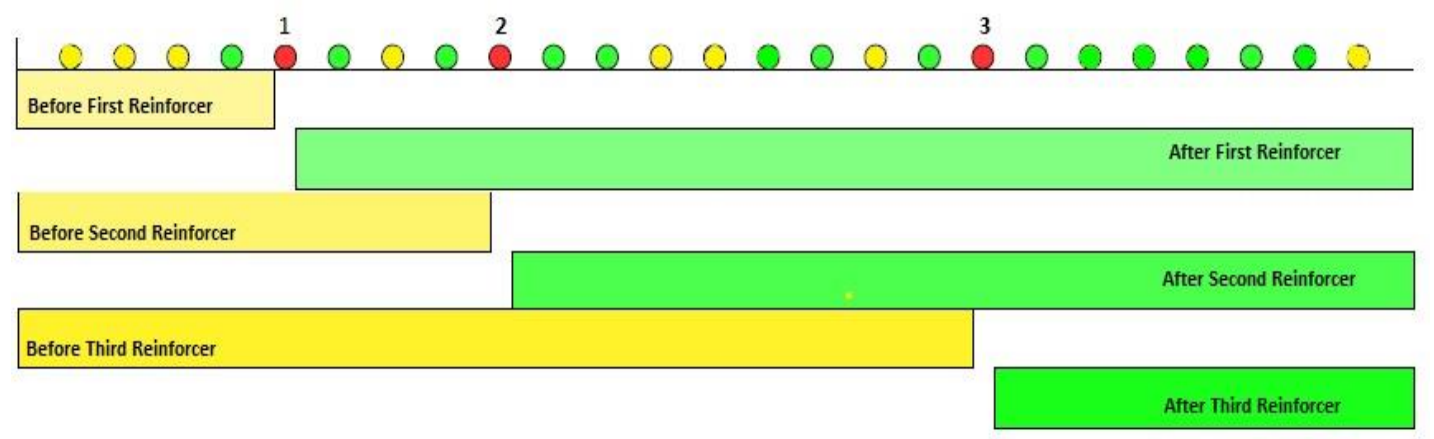

This thesis applies the recounting procedure to measure reinforcement patterns on the same behavior streams used in Sage and Kindermann's (1999) study on children's classroom contingencies. I apply the algorithm using an overall strategy, which identifies reinforcers across all observations and across all focal children, as though observations occur as a single overall series of observations. I re-frame some of Sage and Kindermann's (1999) hypotheses so that reinforcement patterns, and not contingency patterns, are expected across observations. I structure the primary hypotheses around Skinner's definition of reinforcers, and the secondary hypotheses inspired by traditional contingency detection analytic methods used in sequential analysis (Bakeman and Gottman, 1997).

The primary reinforcement hypotheses compare behavior proportions before and after putative reinforcers while the secondary independence hypotheses compare 
behaviors after reinforcers to the overall probability of behaviors (following Bakeman \& Gottman, 1997). I test the primary reinforcement hypotheses by using general estimating equations to account for differences in observations ordered across all focal children when measuring reinforcement at an 'overall' level of analysis. I test the secondary hypotheses by comparing conditional probabilities to overall 'base-rate' probabilities obtained from three different contingency tables from the transformed (i.e. recounted) observations. I use a binomial $z$ test for lagged dependence with the Allison and Liker (1982) corrections for each of these hypotheses. All tests are freely available to researchers via an R Shiny application (Chang, Cheng, Allaire, Xie, \& McPherson, 2016) that I plan to distribute on a university website.

This thesis is structured to provide conceptual and practical overviews, addressing the 'why's' and 'how's of the recounting procedure available in the Shiny web app and used to address all research questions. Accordingly, the literature review outlines theory used to guide observations of reinforcers, and the types of decisions and methods used to structure sequential observational datasets. The next chapter details the purpose of the study, by reviewing Sage and Kindermann's (1999) study on classroom contingencies and demonstrating why this needs to be revisited from a perspective of reinforcers. This chapter introduces the research questions and hypotheses used to re-evaluate Sage and Kindermann's study from the perspective of reinforcers as events that cause change in observed behaviors.

I follow this chapter by the Methods section which has been broken down into three chapters. The first methods chapter provides details of the dataset while the second and third methods chapters provide details on the analysis strategies and their 
implementation in the Shiny web app. This section is followed by the chapter on the Results, which begins by reviewing the analyses strategies and research questions, and then systematically presents the results to each research question by testing each primary and secondary hypothesis. This thesis concludes with a discussion chapter that overviews the strengths and weaknesses of the current study and outlining future directions and next steps for reinforcement detection in naturalistic observational datasets. 


\section{Chapter 2}

\section{Literature Review}

"Any consequence of behavior which is rewarding, or more technically, reinforcing, increases the probability of further responding“" (B. F. Skinner, 1953; p. 345). This quote provides the basis for conceptualizing and measuring naturally occurring reinforcers as proposed in this thesis. While there are many 'post-Skinnerian' theories of reinforcers (e.g., Killeen, 1994; Watson, 1997; Strand, 2000; McDowell \& Caron, 2010; Gallistel, Craig \& Shahan, 2014), these are all deeply rooted in experimental applications of operant conditioning. As the current study focuses on measures of reinforcers based on changes in behavior along the stream of naturalistic observations - and not direct measures of the operant conditioning process - I do not discuss these theories in the literature review. I redirect the interested reader to Appendix A for annotated studies using the principles of operant conditioning in studies of children's classroom behaviors.

By relying on Skinner's original operationalization of reinforcers, the scope of the literature review will be restricted to fundamental behaviorist concepts, which justify and guide the use of observational methods to detect potentially reinforcing events.

Accordingly, I use Skinner's original principles to introduce the fundamental concepts underlying the selection of observable variables and their sequential ordering across time.

The literature review is divided into three sections. I begin the literature review chapter by introducing key terminology from Skinner's learning theory, and when appropriate, their learning theoretic concepts and assumptions. The second section overviews the necessary steps for observing social reinforcers in a stream of naturalistic 
observations and reviews some of the common observational systems and practices used to construct the types of datasets this thesis targets for its proposed reinforcer analysis. Finally, the third section provides a brief conceptual overview of the four elements comprising the meaning of naturally occurring reinforcers - as I use it in this thesis and the analysis.

It is the goal of the literature review to inform potential users of my measure on: a) the background of reinforcers as learning mechanisms, b) the logic behind structuring an observation most appropriate for such an analysis, and c) the reason for conducting a test of reinforcer effects on natural behavior-consequence relations as they occur in natural settings. These three sections provide the necessary information and logic to apply the proposed program to any set of observations - regardless of setting or the nature of variables - but also, argues for the specific case of testing social reinforcers in the manner and settings for which they may most likely act as learning mechanisms in 'real-world' social-developmental processes.

\section{Defining Functional Relations between Antecedent Behaviors and Consequences}

Traditionally, a claim of reinforcement involves the effects of particular environmental events (i.e. target stimulus or focal stimulus) on a particular behavior (i.e. target or focal behavior). Under the assumption that behaviors and stimuli can be meaningfully organized into discrete units, the traditional approach to detecting reinforcers relies on looking for stimuli which relate to changes in the frequency of behavioral units, across time. The following few paragraphs highlight some behavioral assumptions and definitional features, which are critical in a reinforcer analyses, 
especially for sequentially ordered observations.

Target variables. According to reinforcement theory, any behavior is susceptible to reinforcement and any event can be a reinforcing stimulus. Frequency based measures of the reinforcing stimulus and target behavior categories serve as the sole dependent and independent variables of a reinforcer analysis (Skinner, 1953; p. 35). Coming from a mechanistic meta-theoretical perspective (Skinner et al., 2015, chapter 7, p. 4), Skinner's notion of reinforcers focused only on the observable - and does not concern indirect behavioral measures, such as self or other reports of behaviors. As applied to psychological theory, this relegates the mechanism of reinforcers to the behavioral components of psychological processes. Skinner (1953) remarked on the complexity of organizing (and selecting) an appropriate set of physical gestures into a category of behaviors (p.62-68) - and simplified this process by defining classes of behaviors mechanically (e.g., button press, or lever pull). As noted by Bakeman and Gottman (1997) observational studies often categorize behaviors according to extant theory and practical utility (p. 26), a topic I revisit in the following section of this chapter.

Sequential relations between target behaviors and events. Traditionally, Skinner's definition of a reinforcer specifies a sequential criterion for all independent variables to be eligible 'reinforcer candidates' - that is to say, a stimulus can be a putative reinforcer only if it follows the occurrence of a behavior. Accordingly, operant conditioning theory assumes that any target behaviors can be separated into observable 'units' according to what precedes (i.e. antecedents) or follows (i.e. consequences) each event in a behavior stream across time (Skinner, 1953). Skinner made this assumption when defining the occurrence of a reinforcing occasion "...conditioning takes place 
presumably because of the temporal relation only, expressed in terms of the order and proximity of response and reinforcement" (Skinner, 1992, p. 273). Specifically, a target stimulus must follow behaviors in the original order of events to affect future behaviors as a reinforcing consequence. While there is debate amongst contemporary behaviorists as to how direct and immediate a stimulus must be to qualify as a consequence (for a discussion, see Lattal \& Poling, 1981), the predominant line of thought conceptualizes behavioral series in terms of "lags". As this project does not use lags to detect reinforcers, I redirect the interested reader to Sizemore and Lattal (1977) for a discussion on this sequential criterion and its relation to other basic learning concepts, such as contingency.

Functional relations between target behaviors and events. According to operant conditioning theory, an environmental consequence can affect behaviors in one of two ways. When behaviors increase in frequency following a consequence, then this consequence (stimulus) is a reinforcer because it exhibits a reinforcing effect on behaviors. When behaviors decrease in frequency following a consequence, then this consequence (stimulus) is a punisher because it exhibits a reinforcing effect on behaviors.

Skinner (1953) extended this simple classification of effects also to combinations of consequences (i.e. differential reinforcement) and to the effects of either removing reinforcers to decrease behavior frequency (i.e. extinction) or removing a punishing consequence to increase behavior frequency (i.e. negative reinforcement). One issue deserves mention in this regard. In real-world situations, researchers often focus on patterns of reinforcement, or differential reinforcement schedules. I direct the reader to Appendix A for applications of differential reinforcement to modify classroom behaviors). This thesis focuses on identifying the positively reinforcing and punishing 
effects for one observed consequence at a time, and not the effects of combined or cumulative reinforcing events (nor of the removal of a prior consequence).

\section{Observing Social Reinforcement in Natural Settings}

A test of applied psychological theory is whether it is of use in the "real" world. In the case of reinforcers, this test of applied theory concerns 'real-world' environments. Specifically, two features of real-world environments demand consideration when examining reinforcers in applied theory. The first feature comes from Skinner's (1953, 1969) theory of operant conditioning, which rests on the behaviorist assumption that environments control behaviors via sequential interactions, such as antecedent prompts and reinforcing consequences. A second feature of environments is that they control the availability of reinforcers.

The purpose of many naturalistic observations is to determine how likely reinforcers are to occur in the real world, without any-experimental controls or manipulations. Accordingly, it is necessary to select observational settings where reinforcement is most likely available - in order to capture enough observations to test the putative reinforcer for its functional effects on future behaviors. This statement needs little explanation for reinforcers that occur in a highly predictable and controllable way, such as food contingent on lever pulling or other laboratory tasks. For naturally occurring social reinforcers, however, observational researchers must account for a few additional considerations in order to maximize the number of observations of potentially reinforcing events. The following few paragraphs explore these considerations.

Social Reinforcers. A social reinforcer occurs under a scenario: "(when) the 
behaviors of two (or more) people can provide the mediating conditions for future behaviors of both (or more) interactants" (Skinner, 1953; p. 299). Observing social reinforcers requires observing social interactions in such a way that behaviors of one person can be distinguished from the consequences provided by their social partners. This is a matter of designing an appropriate observational strategy, or the set of methods used to sample observations and translate these observations into a dataset suitable for statistical analyses, via a coding scheme. Accordingly, to analyze social interactions for social reinforcers it is necessary for naturalistic observational strategies to: a) sample situations in which social interactions are most likely and b) decompose the interaction into discernable behavior-consequence patterns. In Skinner's words:

We may analyze a social episode by considering one organism at a time. Among the variables to be considered are those generated by a second organism. We then consider the behavior of the second organism, assuming the first as a source of variables. By putting the analysis together, we reconstruct the episode. The account is complete if it embraces all the variables needed to account for the behavior of the individuals (Skinner, 1953; p. 304).

\section{Coding social behavior in the natural series of events. A continuous event-}

coding sampling strategy lends itself most directly to the analyses of behaviorconsequence ordered pairs. Bakeman, Deckner and Quera (2005) describe an event (or categorical) coding system as one which uses a set of codes to capture all behaviors and events that occur during an observational 'episode' (p. 397). Specifically, a continuous event sampling procedure captures each behavior and consequence as the stream of 
behaviors unfolds across time (Farmer \& Schoenfeld, 1966; Lattal, Abreu \& Rodrigues, 1997). This contrasts with event interval sampling methods used in early behaviorist studies (see Kazdin \& Klock, 1973; Pfiffner, Rosen, \& O’Leary, 1985). These sampling strategies divide an observation into equally spaced intervals a-priori and fit one behavior into each 'unit'. When the goal is to identify natural reinforcing social behaviors, as in the current case, it seems plausible to consider observations using either strategy - if the strategy records the behavior and events of interest as they unfold in their natural and ordering across time.

Common social interaction observations systems. Observational datasets oftentimes include more information than is necessary for a simple reinforcer analysis. This is especially true for observations of social interactions. According to Altman (1974), these observational datasets often use process-oriented behavior systems and contain information regarding: 1) the source of antecedent behavior (i.e., 'actors'), 2) the source of social consequences (i.e. partners), 3) the order of events (i.e., antecedents and consequences), and 4) an indicator of time or duration of the events (p. 236).

Observational datasets using these coding systems have adequate information to reconstruct 'the social episode' for the analyses of socially reinforcing interactions.

Process-oriented behavior systems usually use a focal person sampling strategy to obtain a single behavior record for each participant in an observation (Baltes, 1988). This strategy requires an observer to focus on the behavior of one person at a time and to register all of this person's social partner's behaviors as they interrupt the focal person's stream of behaviors (see Soloman \& Whaler, 1973; Andrews \& Kozma, 1990). This sampling strategy results in a dataset containing a stream of behaviors as well as 
identifier for the focal person's and their social partners. It retains the natural order of events as they occur during the observations

One example is the Interpersonal Process Coding System (Rusby, Estes, \& Dishion, 1991). It uses a five-digit code for each focal person's record of observations; where each digit of the behavioral code represents: a) the focal child, b) the behavior, c) its status (e.g., initiation or response), d) the ID of the social partner and e) the duration of behavior (p. 6). Antecedent consequence ordered pairs are identifiable when partner response codes (usually as categories of partners) follow a child's behavior in the natural order of events. If partner ID's are included, a reinforcer analysis of these datasets may easily recode partner's identification variables into categories (e.g., parents, peers, etc.) thus allowing for a continuous stream of behavior-consequence observations across all focal participants.

The Interpersonal Process Coding System contrasts with systems using the behavior-consequence contingency as a unit of analysis. Instead of independently recording the behaviors of focal person's and their social partner behaviors, Good and Brophy's (1970) scheme for observing child-teacher interactions, for example, records the ordered behavior-consequence pair as the unit of analysis. This system uses a spreadsheet format with variables for the onset and offset (i.e., duration) of behaviors involved in the behavior-consequence pair. A reinforcer analysis of these datasets would require a substantial amount of data-preparation to remove behaviors from the contingency codes, in order to look for future change in the antecedent focal child's behavior.

The aforementioned coding systems have received substantial use in the study of 
social influences (Dishion, Duncan, Eddy, Fagot \& Fetrow, 1994; McDowell \& Caron, 2010), in families and educational settings (e.g. Baker, 1999). Particularly, these systems are suited for answering questions regarding the probabilistic contingency - or the probability that a specified consequence occurs given the antecedent behavior (Kindermann \& Skinner, 1988, p. 69)². Using a statistical program (e.g. GSEQ; Quera \& Bakeman, 1998), this analysis strategy 'extracts' all target behaviors from the ongoing series of observations. This subset of behaviors serves as the 'condition' for measuring the probability of a target consequence - and a claim of contingency is made by comparing this probability to that of the consequence occurring (unconditionally) after any behavior in the observations. This analysis addresses the availability of consequences within certain environments but not the functional effects on future behaviors of individuals. Consequently, the complicated structure of these observational systems is suitable for contingency analyses, but may prove more challenging for a reinforcer analysis of ongoing behavior change.

An alternative way an observational system can represent the potentially reinforcing exchange is to use a set of rules that dictate how features of the exchange (e.g., duration, onset, offset, initiation, response, etc.) are embedded into the coding of behavior streams. As Bakeman and Quera (1995) remark, such coding rules are often necessary when an observation stream contains a mix of mutually exclusive and exhaustive codes. Sage and Kindermann's (1999) study on classroom contingencies, for example, used separate sets of codes for the behaviors of focal person's behaviors and

\footnotetext{
${ }^{2}$ Note, that according to Gewirtz (1997, p. 122), the term probabilistic contingency is typically used in reference to non-experimental contingencies. Gewirtz notes that this term is typically used to imply an ordered and dependent relation between stimulus and response (e.g., S-R, R-S, S-S).
} 
that of their social partners. In this scheme, the transition from one ordered pair of codes to another (e.g., behavior-behavior, behavior-consequence), marks the onset and offset of behaviors. When used in conjunction with rules for re-coding behaviors, such a scheme allows for a frequency-based contingency analysis as well as for the analyses of reinforcer effects - with minimal requirements for restructuring the observations - so long as the coding rules are used in the interpretation of results and the behavior category system consists of mutually exclusive and exhaustive categories. The proposed program will accept datasets using either strategy for re-constructing the social episode within the stream of naturalistic observations.

\section{Real World Reinforcement Patterns}

Since the early 1970's, a small strand of social-developmental studies attempted to develop detection methods for naturally occurring reinforcers using observational and statistical methods. These studies do not typically rely on naturalistic observations - these studies (e.g., Patterson, 1974; Snyder \& Patterson, 1986; Strand, Wahler, \& Herring, 2001) applied detection methods to reinforcers as they occur during short-term observations within quasi-experimental settings. This is a way to control the occurrence of behaviors as well as of social reinforcements. I mention this line of research to illustrate two applied dimensions of environmental control as it pertains to the study of reinforcement in natural settings.

The first dimension of environmental control is that of setting. Applied behavioral interventions study 'real-world' reinforcers - where 'real-world' denotes that the demonstration of reinforcers occurs in non-laboratory settings, such as schools or clinics. 
These studies test reinforcers between partners who share naturally occurring bonds, such as teachers and their pupils, in natural school and classroom settings, but do so by controlling the immediate behavior consequence contingencies between a focal person's behavior and the consequences provided by their social partners. One may call such studies naturalistic simulations. Typically, a researcher instructs a social partner to provide potentially reinforcing consequences systematically, rather than relying on social partners' natural tendencies. Appendix A illustrates some of these strategies.

The second dimension is that of relations. There are three components to the natural relations between behaviors and their social consequences. These components are: 1) the social/emotional bonds between partners, 2) the immediate behavior-consequence contingency, and 3) the functional effects a consequence has on future behaviors. Applied behavioral interventions, for example, may manipulate behavior-consequence relations between real-world partners in real-world settings. In their (1973) study on classroom reinforcers, Soloman and Whaler did just this, by instructing carefully selected children to socially praise or ignore the on or off-task behaviors of their disruptive peers. This study took place in a real-world classroom and only changed the immediate behaviorconsequence relations between children to determine the function of these consequences on future behaviors (see Appendix A for details).

The quasi-experimental social-developmental studies have studied the natural reinforcement relations between social partners. The bonds between these partners occur naturally, such as in the case of parents and siblings. These studies manipulate the setting by observing interactions in laboratories or according to structured tasks. A common goal of these methods is to heighten the occurrence of naturally low frequency behaviors, such 
as violent or aggressive behaviors. Early laboratory studies (e.g. Azrin \& Lindsley, 1956; Patterson \& Anderson, 1964; Titkin \& Hartup, 1965), have looked for functional differences in the effects of social approval from children sharing 'self-selected' natural relations, such as friends or peer groups, but did so by manipulating behaviorconsequence relations via experimental instructions. Finally, those studies using contingency detection methods, such as Sage and Kindermann (1999), have studied differences in self-selected social partners under real-world settings but focused on the immediate behavior-consequence sequential contingency, without testing the assumption that these contingencies are indeed reinforcing.

\section{Conclusion}

The goal of the current project is to detect reinforcer effects observations. The proposed program offers a strategy for assessing all four features of naturally occurring reinforcers: 1) naturalistic setting, 2) naturalistic social-partner relations, 3) naturally occurring sequential relations, and 4) naturally occurring functional effects of consequences on future behaviors. The program aims to facilitate analyses in naturalistic datasets studying natural behavior-consequence relations in their naturally occurring social and physical environments.

This project draws inspiration from the idea that examining antecedentconsequence probabilistic contingency analysis of putative reinforcers (Bakeman \& Quera, 1995) can benefit from the analysis of actual reinforcement effects. These combined analyses can rule out behavior-consequence relations that occur contingently with probabilities greater than chance but have no influence on the occurrence of future 
behavior. The goal is to demonstrate a novel method to detect naturally occurring reinforcers under each of the real-world features of naturally occurring social-interaction episodes. 


\section{Chapter 3}

\section{Purpose of the Study}

The current study has as its primary goal to develop a reinforcement detection algorithm that allows researchers to identify patterns of naturally occuring reinforcement in observational datasets of naturalistic sequential observations. It is estimated that about a hundred or so studies exist that use naturalistic sequential observations of this type. The strategy is based on Skinner's (1938) definition of reinforcement: "...any consequence of behavior which is rewarding, or more technically, reinforcing, increases the probability of further responding" (B. F. Skinner, 1953; p. 345). A second goal of the project is to introduce a flexible web-application to accommodate the many types of observational data sets. This study uses the data from Sage and Kindermann (1999) study on classroom contingencies in order to test for reinforcers and punishers. I adapt the research questions and hypotheses of Sage and Kindermann's study to fit reinforcement patterns rather than contingency patterns. Because my research questions concern reinforcement (and not contingency), I draw from the experimental literature as support for potential reinforcement effects of given behaviors. 
Figure 2. Reinforcement detection measure (a.k.a. recounting procedure) (Figure 1 reprint)

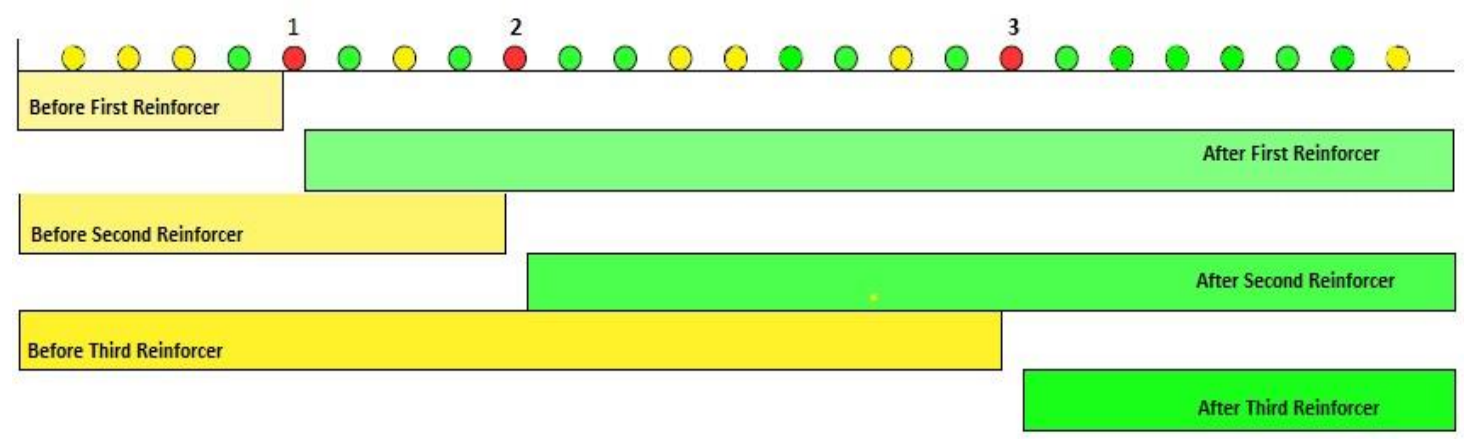

This chapter is divided into four sections. The first section provides an overview of Sage and Kindermann's (1999) research questions and results concerning behavioral contingencies. The second section takes a closer examination of the difference between contingencies and reinforcers, specifically, focusing on what it means to re-examine classroom contingencies as potential reinforcers. The third section builds from these concepts and formally states the research questions and hypothesis of the current study. Finally, I conclude the chapter by priming the reader for the methods section of the thesis, which takes a more general case for examining reinforcers by introducing general methods and software that can re-examine contingencies as reinforcers, for any set of observations.

\section{Classroom Contingency Patterns}

Sage and Kindermann's (1999) study on classroom contingency patterns asked a simple question: do classroom behaviors depend on their social consequences? A prime example is of a disaffected child and their off-task behaviors. Do the child's off-topic jokes depend on the approval they get from their classmates? Does this child's on-task behavior depend on whether the teacher gives an approving response? 
To address these questions, Sage and Kindermann observed a typical classroom for a period of two weeks and then analyzed these data for sequential contingency patterns, using lag-sequential analysis (see Bakeman \& Quera, 1995). Lag sequential analyses refers to a suite of these techniques which are used to determine the probability of one observation following another in the stream of observations. These are oftentimes used in the analysis of 'probabilistic contingency patterns' taken from the stream of naturalistic observations (Ator, 1999). Because the classroom comprises a variety of behaviors and activities, Sage and Kindermann focused on a few behavior-consequence patterns, namely, that On and Off-task behaviors would both be followed by social approval (e.g., laughter or praise).

To account for context dependencies, Sage and Kindermann (1999) differentiated behavioral contingencies amongst classroom social partners. These social partners provide different social contexts, suspected to provide different 'mediating conditions' for reinforcement processes. Specifically, Sage and Kindermann focused on three categories of social contexts: peer group members (or close affiliates), other classmates, or the classroom teacher. The peer group context was expected to be the main context for which reinforcement occurs for On and Off task behaviors, while the teacher was expected to be the primary source of reinforcement for On-task behaviors. Students were classified as having low or high engagement levels, as were their peer groups.

Contrary to expectations, Sage and Kindermann's (1999) study did not find the clear support for differences between peer group members and non-members. For OnTask behaviors, Social Approval was a significant consequence within highly engaged peer groups as well as in interactions with their teachers. In groups of low engaged 
students, there was only support for teacher's approval for On-Task behaviors. Peers (group members or other students) were not found to be significantly related to different contingencies for On or Off-Task behaviors.

\section{Contingency vs Reinforcement in Classroom Observations}

While compelling in its own way, Sage and Kindermann's contingency analysis does not directly speak to reinforcer effects of Social Approval or any of the other behaviors investigated in their study. Instead of directly testing how behavior changes in a stream of observations, lag-sequential analyses test whether the underlying antecedentconsequence sequential structure allows for a better prediction of target behaviors, than not knowing this structure at all (Bakeman and Quera, 1995). In terms of Sage and Kindermann's (1999) study, all lagged sequential hypotheses concerned predicting putative reinforcers (e.g. consequences) for targeted antecedent behaviors. This same technique can be applied to test the reverse, that is, predicting behaviors as consequences from potential reinforcers as antecedents.

No matter how one looks at a lag-sequential analyses, the standard approach is limited from a reinforcement perspective, namely, in that it does not look for changes in the dependent variable across the times that reinforcement occurs. This strategy assumes that 'probabilistic contingencies' are necessary and sufficient criteria for sifting reinforcers from other behaviors. This is assumption may not always be the case. On the contrary, a putative reinforcer may have no systematic correlations with behaviors under real-world conditions. In this way, the only way to test a reinforcer is directly, that is, by its effects on future behaviors, regardless of the probability of the immediate behavior- 
consequence contingency.

The dependent variable in a lag sequential analysis is a specific observation at time $t+l$ (conventionally called the consequence) and the independent variable is a specific observation at time $t$ (conventionally called the antecedent), where $l$ is a lag. For the analysis of behavior-consequence contingencies, as in Sage and Kindermann's (1999) study, hypotheses were framed in terms of lag 1 contingencies, where the consequence is the potential reinforcer. Note, that this can be analysis can reframed so that the consequence is a behavior (and the antecedent is the consequence), this approach is discussed in more details in Appendices G and H. For illustrative purposes, I focus on Sage and Kindermann's approach for testing the consequence as reinforcers.

Figure 3. Conceptual depiction of contingency detection via lag-sequential analysis

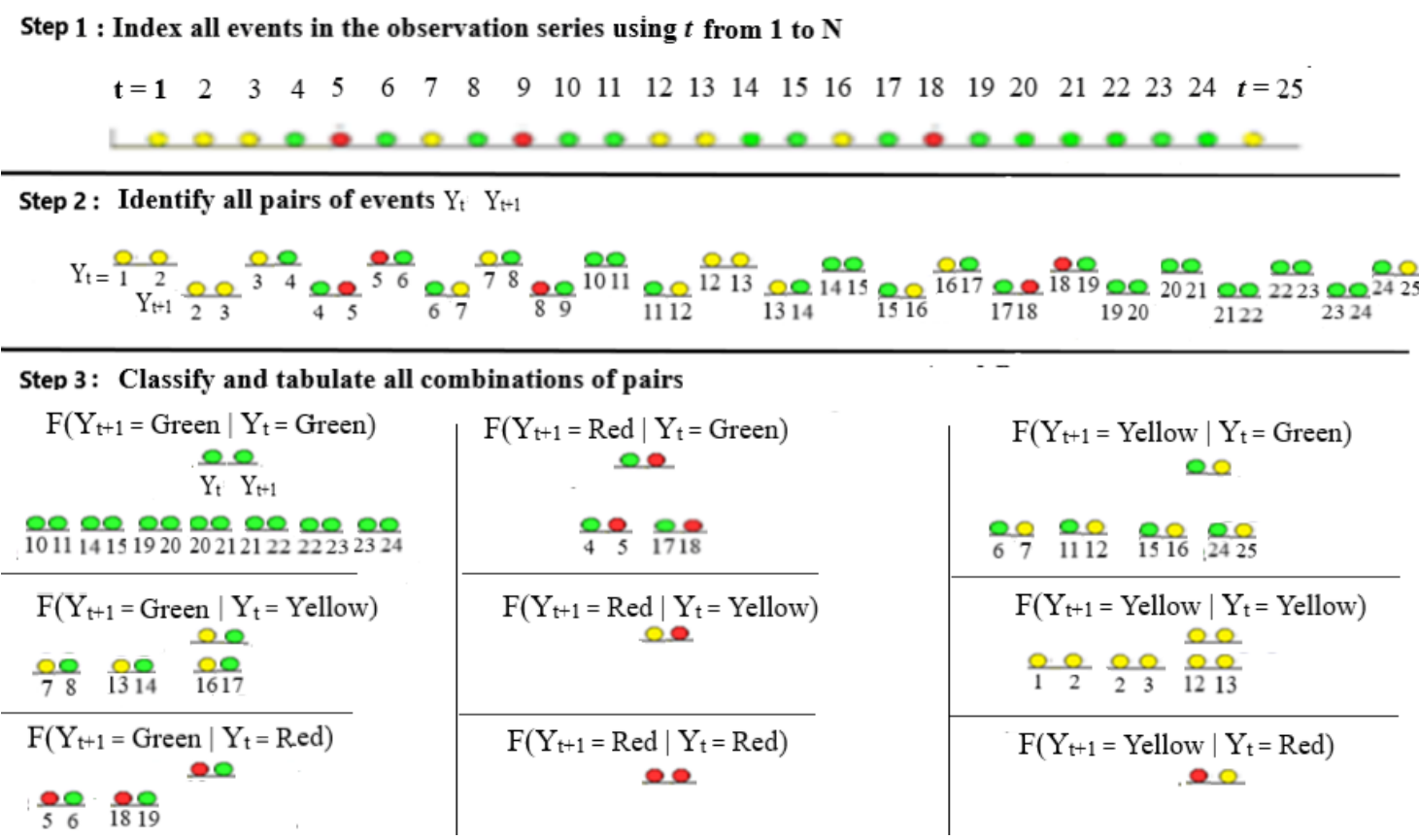

Figure 3 shows a three-step conceptual process of identifying and sorting all lagged pairs in the stream of observations. The first step is to identify the order of all 
observations using an index $t$ (see Chapter 5 for an alternative strategy for indexing with lags). The second step is to pair each observation at time $t$ with the next observation at $t+1$. Note, as is discussed latter in this chapter and throughout the thesis (see Chapters 5 and 7), this method naturally introduces dependency by resampling the same observations. Thus, the consequence in the first pair of Figure 3 (e.g., observations 1 and 2) becomes the antecedent at for the second pair (e.g., observations 2 and 3). Finally, the third step is to tally the number of observations for each pairing and represent this in a contingency table; where the rows show the dependent variable at time $t$ and the columns show the dependent variable at time $t+1$.

Table 1. All lag-one conditional frequencies from Figure 1

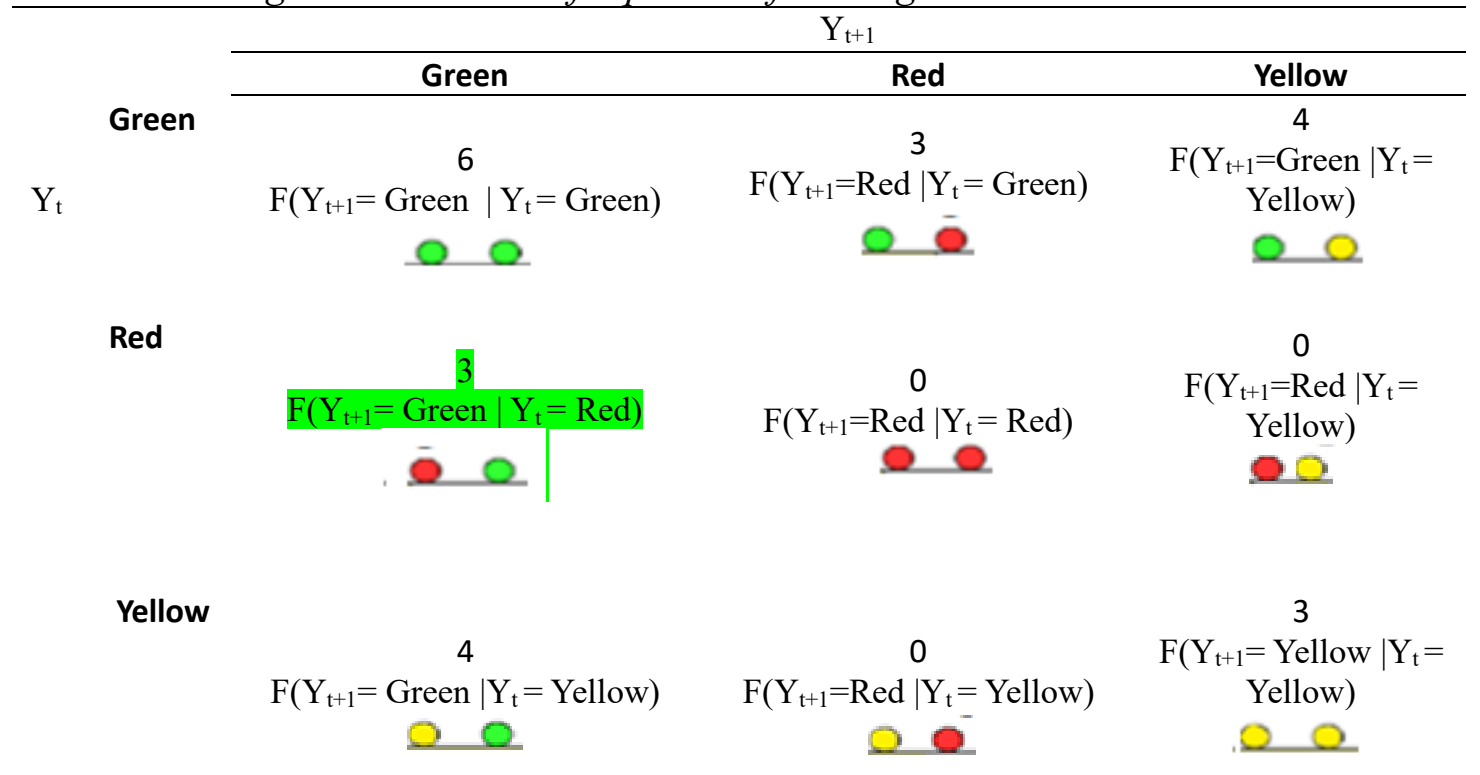

Table 1 shows the counts of all pairs from Figure 3 (see Chapters 5 and 8 for a demonstration of how this table can be broken down even further by summing across the non-target behaviors for the antecedents and consequences). This full $3 \times 3$ table is then used as the basis for computing the necessary proportions to test whether the target 
consequence is more likely to follow the target antecedents, than any other event in the stream of observations.

After constructing the contingency table for lagged dependence, specific hypotheses can be tested with the Allison and Liker $z$-score (1982). Originally proposed by Sackett and colleagues (1979), this test was latter adapted by Gottman (1980), and then corrected by Allison and Liker (1982), who noted an error in the original calculation for the standard errors. The Allison and Liker $z$-score is a statistical test for sequentially dependent observations. It compares lag-conditional proportions and overall base rate proportions and adjust for sequential dependence by its standard error of the difference. In terms of Sage and Kindermann's study, it was used to test the target consequence given a target antecedent by the overall proportion of times the target consequence occurs. Equation 1 shows the Allison and Liker $z$-score.

Equation 1. Allison and Liker (1982) binomial z-score for lag analysis

$$
z_{1}=\frac{\operatorname{Pr}\left(Y=\text { Target }_{t+1} \mid x=\text { Target }_{t}\right)-\operatorname{Pr}\left(Y=\text { Target }_{t+1}\right)}{\sqrt{\frac{\operatorname{Pr}(Y=\text { Target })(1-P r(Y=\text { Target })(1-P(Y \mid x=\text { After })}{(N-1) * P(Y \mid x=\text { After })}}}
$$

Reframing Contingencies to Reinforcers. From a reinforcement lens, a closer look at the process depicted in Figure 3 reveals an important missing feature, namely, contingency does not indicate when a consequence is likely to occur along the series of observations. It is clear from the figure that more green circles occur after red circles, towards the end of the stream of observations. This pattern indicates reinforcement occurs but cannot be detected by the lag-one contingency analysis for the consequence. This is 
because the lag-one contingency analysis computes the dependent variable lagged at one direction at a time (e.g., DV lagging behind or ahead of the IV), and at one step (e.g. lag forward one step, two steps, etc.). A contingency analysis can possibly detect this pattern by examining multiple lags (see Gottman and Roy, 1990, chapter 4 for a comprehensive discussion), and combining a 'lag-forward' and 'lag-backward' approach (see Appendix $\mathrm{G}$ for details), but these strategies fall outside the scope of this thesis, as explained latter in Chapter's 5 and 7.

This thesis presents an alternative to the contingency detection algorithm that specifically accounts for where behaviors are more likely to occur along the stream of observations. To do this, I present a reinforcement detection strategy that can be conceptualized as extending a lag analysis in both directions, and across behaviors in the stream of observations. While the technical details of the reinforcement detection procedure are reserved for the next chapter, it is easy to conceive of how a contingency hypothesis can be reframed from this reinforcement perspective, and how this reframing may shed new light on past studies and observations.

Sage and Kindermann's (1999) study is a good springboard for reframing contingency detection in terms of detecting reinforcers. Their study looked at differences between social partners in social contingencies, for example. One such question was whether Social Approval more likely to immediately follow On-Task behavior for children's peer group interactions than interactions with other classmates? If we are to remove the peer group component and look specifically at reinforcers, we can reframe this question as whether On-Task behavior is more likely to occur following Social Approval than it is before Social Approval? In this thesis, I rephrase the research 
questions from Sage and Kindermann's study in terms of proportions; without reference to any other consequences nor the contingency between the target behavior and consequence.

\section{Research Questions and Hypotheses}

The main hypotheses of this thesis test the assumptions of Kindermann and Sage's (1999) study, namely, that naturally occurring Social Approval in the classroom reinforces On-Task behaviors and Off-Task behaviors, and that Disapproval punishes Off-Task behaviors. My research questions go beyond this study, and each set of hypotheses concern the effect of the consequence on behaviors. Each research question contains a primary hypothesis which posits that the probability of a target behavior will be greater after reinforcers and will be greater before punishers, than before these behaviors. The primary hypotheses are based on Skinner's (1953) definitional criteria of reinforcers, which states that "... any consequence of behavior which is rewarding, or more technically, reinforcing, increases the probability of further responding (B. F. Skinner, 1953; p. 345)".

Additionally, each research question contains a secondary hypothesis which tests for an association between the effect of a putative reinforcer and a behavior by comparing the overall proportion of the target behaviors to proportion of target behaviors occuring after reinforcers. These secondary hypotheses draw inspiration from lag-sequential analyses, and the conceptualization of the reinforcement detection procedure as an extension of this analysis strategy. All secondary hypotheses are tested three times, across three different contingency tables based on the recounted stream of observations (Chapter 
5 for an elaboration of these tables). Finally, I frame each hypothesis in terms of an 'overall'strategy, that is, where behaviors are classified as before or after reinforcers along one 'overall series', across all focal children and their social partners, and observational episodes. In this way, each hypothesis is expressed as if all observations belong to a single person across a single observational episode.

Research Question 1: Reinforcers of Classroom Behaviors. Does the occurrence of direct social approval act as a reinforcing consequence for a child's behaviors?

Social affirmation has received the most experimental attention as a positive reinforcer for a variety of classroom behaviors. To demonstrate these effects in classrooms, researchers have drawn upon single-subject experimental studies in order to construct classroom interventions and behavioral management strategies for use in general education classrooms (for a comprehensive discussion see Honer, Carr, Halle, McGeem, Odom, \& Wolery, 2005). Using the teacher to 'distribute' praise, a large number of class-wide single-subject experiments and applied classroom implementations have shown that social approval is generally an effective social consequence for increasing engage classroom participation and performance (for reviews, see Partin, Robertson, Maggin, Oliver, \& Wehby, 2009 ; Hutchings, Martin-Forbes, Daley, \& Williams, 2013). That is, despite variation behavior-consequence delivery ratios, following specific behaviors, or delivered in public or private settings, Social Approval is an effective means of increasing desired classroom behaviors (Houghton, Wheldell, \& Sharpe, 1990 ; Sutherland, Wehby, \& Copeland, 2000 ; White, 2010 ; Kazdin \& Klock, 1973; Wheldall, Bevan, \& Shortall, 1986 ; Broden, Bruce, Mitchell, Carter, \& Hall, 
1970 ; Blaze, Olmi, Mercer, Dufrene, \& Tingstrom, 2014). Consequently, naturalistic observations have shown that these relations hold outside intervention contexts, using measures of correlation between children's active and overt learning behaviors and various types of praise for on-task behaviors (see Jenkins, Flores, \& Reinke, 2015 for a review).

Hypothesis 1: Occurrences of social approval will lead to an increase in Active On-Task behaviors when treating the entire Sage and Kindermann (1999) data set as a single uninterrupted series of events.

Stated differently, the overall probability of On-Task behavior will be lower before events of Social Approval than they will be afterwards. A primary test of this hypothesis uses general estimation equations on the recounted series to account for the dependency between observations while testing the difference between the probability of On-Task behavior before after Social Approval. A secondary analysis will use a version of the $z$ test for lagged dependence to test the difference between On-Task behavior after Social Approval and On-Task behavior across all observations.

Justification of Hypothesis 1. This was a significantly contingent pattern in Sage and Kindermann (1999). Across all focal children and their social partners, those children who were visibly engaged in their learning were most likely to receive overt and direct recognition for their efforts. As experimental studies have shown that this type of recognition can lead to increases of on-task behaviors, it stands that this type of reinforcement pattern will also appear in the everyday stream of classroom behaviors.

Hypothesis 2: Occurrences of social approval will lead to an increase in Active Off-Task 
behaviors when treating the entire Sage and Kindermann (1999) dataset as a single uninterrupted series of events.

Stated differently, the overall probability of Active Off-Task behavior will be lower before events of Social Approval than they will be afterwards. A primary test of this hypothesis uses general estimation equations on the recounted series to account for the dependency between observations while testing the difference between the probability of Off-Task behavior before and after Social Approval. A secondary analysis will use a version of the $z$ test for lagged dependence to test the difference between Off-Task behavior after Social Approval and Off-Task behavior across all observations, across the three contingency tables representations of the recounted stream of observations.

Justification of Hypothesis 2. This interaction pattern is at the heart of 'deviancy training' or social influence towards rule-violating behaviors (Dishion, Piehler, \& Myers, 2008). In their seminal 1999 study, Dishion and colleagues demonstrated that behaviors typical of social approval (e.g. laughter) is most likely to follow 'rule-violating' talk or behaviors in 'deviant' adolescent friendship dyads, compared to 'normative' friends. Moreover, the frequency of these patterns was predictive of future legal violations, ten years later. While these patterns may occur with less frequency under the teacher's presence, it is still reasonable to assume that Social Approval from disaffected peer group members can act as a reinforcer for Off-Task behaviors in the classroom.

Research Question 2: Punishers of Classroom Behaviors. Does the occurrence of direct Disapproval act as a punishing consequence for a child's Off-Task behaviors?

According to reinforcement theory, social consequences can determine whether 
behaviors continue or stop under a given setting or under given environmental conditions. In classrooms, a child's disruptive or overt Off-Task behaviors, are one of the most heavily reported 'problems' teacher's face in managing their children (Partin, Robertson, Maggin, Oliver, \& Wehby, 2009). Intuitively, it stands that direct Disapproval would effectively reduce a children Off-Task behaviors, especially, when this Disapproval occurs in public and comes from teachers. While the effectiveness of Disapproval as a punisher has received most evidence in parent-child interactions outside of classrooms (e.g., Patterson, 1972; Strand, Wahler, Herring, 2001), its appeal as a punisher intuitively extends to classroom interactions.

Hypothesis 3: Occurrences of direct Social Disapproval will lead to decrease in active Off-Task behaviors when treating the entire Sage and Kindermann (1999) dataset as a single uninterrupted series of events.

Stated differently, the overall probability of Active Off-Task behavior will be higher before events of direct Disapproval than they will be afterwards. A primary test of this hypothesis uses general estimation equations on the recounted series to account for the dependency between observations while testing the difference between the probability of Off-Task behaviors before and direct Disapproval. A secondary analysis will use a version of the $z$ test for lagged dependence to test the difference between Off-Task behavior after Disapproval and Off-Task behavior across all observations, across the three contingency tables representations of the recounted stream of observations.

Justification of Hypothesis 3. An interaction pattern that direct Disapproval followed Active Off-Task behavior was not a statistically significantly contingency pattern in Sage and Kindermann (1999). While this can easily lead to the expectation of 
non-significant reinforcement patterns, it can also lead to the opposite expectation - that punishing events may occur 'vicariously' in classroom settings. In this way, a child's future behavior receives influence by watching other children receive reprimands for negative or inappropriate behaviors. As an example, a specific child may engage in OffTask behavior to be met with teacher's direct Disapproval (one event) -- after observing this exchange, other children may be less inclined to engage in actively disruptive behaviors. It is for this reason I do not want to state an expectation of non-significance. In fact, it may be that the ability to detect functional effects of non-contingent social consequences may become a primary contribution of this project.

\section{Outline of Methods}

This study introduces a method used to identify reinforcement patterns in naturalistic observations. Specifically, this method assumes that naturalistic observations are typically not structured in a way that easily accommodates comparing proportions of behaviors before and after reinforcers, but they can be reorganized to accommodate such goals. Alternatively, the proposed method is intended for any set of consecutive codes, in which one code serves as a putative reinforcer and another code as a target behavior (to be reinforced). This strategy has two components; a 'classification' piece and a 'measurement' piece. The 'classification piece' is used to identify observations as occuring 'before' or 'after' reinforcers, for 'targets' or 'non-targets'. This preserves the structure of the observations and labels observations according to reinforcers (e.g., before or after). The 'measurement' piece is used to calculate and compare proportions, while accounting for dependence in repeated observations. 
The reinforcement detection strategy fills a gap in the social interaction literature, whereby studies assume reinforcement patterns using lag-sequential analysis (see Bakeman \& Gottman, 1997; Sackett, 1979 for traditional analytic approaches). The current study current project adds one more step to these traditional approaches; by grouping all observations before and after reinforcers. In this way, the end goal of the project is to demonstrate and distribute a strategy for detecting reinforcing events along any set of observations. Additionally, this thesis proposes a web-accessible software application that implements this detection strategy, for use on any observational dataset for research studies. This thesis demonstrates this strategy and web-application on a set of classroom observations collected by Sage and Kindermann (1999).

The methods section is divided into three chapters; each chapter describes an aspect of the applet's design and reinforcement detection strategies. The first methods chapter describes the original purpose, setting and sample, observational strategy, and coding categories, used to construct the specific data set (Sage \& Kindermann, 1999) used in the current study. I describe the variables and the repeated measurement structure of the original data-file. I conclude this part with an example that illustrates the translation of the observational methodology (including the coding rules) to a dataset ready for a reinforcer analysis. The remainder of this chapter uses examples inspired by this dataset to illustrate the strategy developed to detect reinforcers.

The second methods chapter details the strategies used to classify observations by reinforcement patterns and test these patterns for statistical significance, following all primary and secondary hypotheses. In doing so, this chapter introduces the reader to a language used throughout the thesis to refer to the transformed (i.e. recounted) 
observation series. Figure 4 (a repetition of Figure 1), shows the process of transforming an observation series using an 'overall strategy'; in which an observational dataset is restructured to uncover potential reinforcement patterns without regards to the repeated observations within separate focal individuals or days of observations. The final product of the recounting procedure is a restructured dataset (called the recounted datafile) that can be used directly in count-based data analysis methods. This chapter concludes by overviewing the use of general estimating equations (Liang \& Zeger, 1986) for binomial responses and Allison and Liker $z$ - tests (Allison \& Liker, 1982) for summary contingency tables, used to address all primary and secondary hypotheses, respectively.

Figure 4. Overall reinforcement detection measure (Figure 1 reprint)

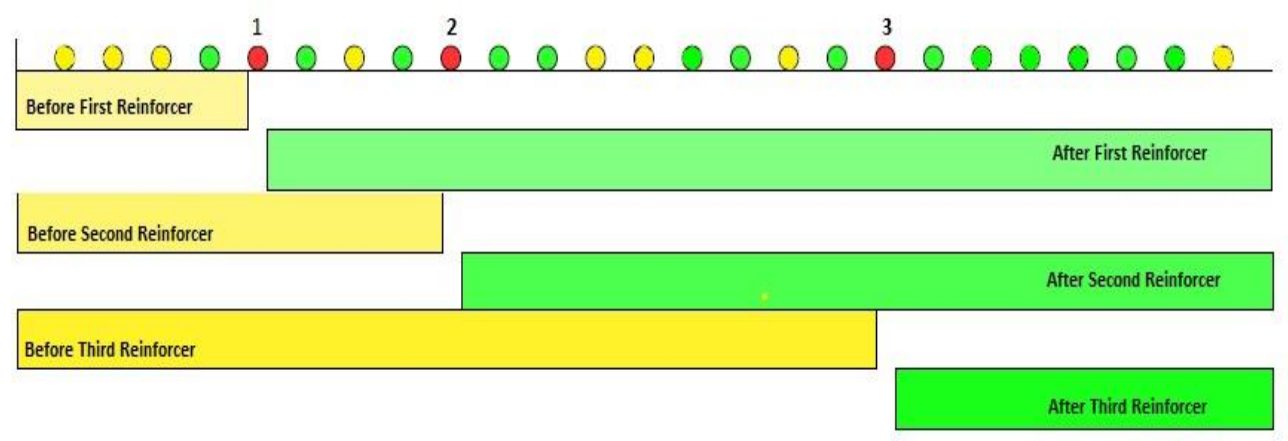

The third methods chapter concludes the discussion on methods by discussing the implementation and distribution of the reinforcement detection strategy. This chapter elaborates upon the concepts covered in the second methods chapter (e.g., recounted data-file, contingency tables, etc.), and specifically, the statistical techniques included in the Shiny web-app package (Chang, Cheng, Allaire, Xie, \& McPherson, 2016) using the R language (R Core Team, 2016). This chapter focuses on the layout of the web-applet 
and describes its features. 


\section{Chapter 4}

\section{Methods 1: Description of the Dataset}

The current study investigates naturally occuring reinforcement processes using the dataset from Sage and Kindermann's (1999) observational study on classroom interactions. Sage and Kindermann's dataset comprise a two-week long series of classroom observations, balanced across lesson subjects, and with adequate observer reliability. The study used this dataset to examine sequential contingency patterns, namely, patterns in which potential reinforcers, such as social approval, were more likely to follow behaviors of an individual than could be expected by chance (see Bakeman \& Gottman, 1997). Specifically, the study examined whether putatively reinforcing contingencies from the members of a child's self-selected network of peers, classmates, or the teacher occurred with a higher likelihood than could be expected by chance.

The current study goes further and applies the reinforcement detection strategy on Sage and Kindermann's (1999) dataset to determine whether these contingent consequences are reinforcers or punishers of children's behaviors (see Chapter 2 for details). To give an example, approval contingencies for on-task behaviors are ostensibly supportive and reinforcing, even for children who are disaffected in class. This positive reinforcing effect may be especially noticeable when approval comes from a member of that child's well-adjusted peer group. When coming from teachers, especially in front of classmates, this same act of social approval may have opposing punishment effects, and function more as a 'kiss of death', by embarrassing students and making active 
participation less likely in the future (e.g. Tal \& Babad, 1999) ${ }^{3}$. The current study uses Sage and Kindermann's (1999) dataset as a basis for testing these potential reinforcer effects from known contingencies.

Sample and setting. This dataset contains 12,043 behavioral observations of focal child behaviors and partner consequences (coming from a member of that child's independently identified peer group, from a non-group classmate, or from the teacher). Of the 25 children in the classroom, 22 agreed to participate in the original study. The observations were collected across 10 consecutive school days in a small mixed $4^{\text {th }}$ and $5^{\text {th }}$ grade classroom located in the Pacific Northwest. Six trained observers participated in the study (following four months of observer training; inter-observer reliability was above Cohen's $\kappa=.8)$.

The classroom teacher organized learning activities according to an open learning structure - where learning materials were distributed across various teaching formats, such as group projects, traditional lecture. For example, children were usually not in preassigned seats in the classroom but grouped themselves according to ongoing projects, even during lecture times. Observations were distributed about evenly across all learning activities (Math/Science, Language, Social Studies) as they occurred in the uninterrupted daily routines.

Observational strategy. All observations were audio re-corded using microphones and headsets. A focal person observation strategy (see Baltes, 1988) was used to record natural social interactions. On each day of observations, children were

\footnotetext{
${ }^{3}$ I re-direct the interested reader to Appendix A for more information on this phenomenon, and how it may or may not alter the reinforcing function of teacher praise.
} 
assigned to a three-minute observation schedule, twice per day, where the order was of the observation segment was randomized daily. To ensure reliability, two observers were present during each observation session (reliability checks were performed on approximately $10 \%$ of the total observations according to pre-determined times in the random schedules). The observed classroom activities were balanced across mathematics and science, reading/writing, and social studies.

Coding Scheme. The coding scheme comprises two sets of mutually exclusive and exhaustive categories, one set for focal children's behaviors (see Table 2), and a second set for the consequences provided from their social partners (See Table 3).

Table 2. Focal children behavior codes

\begin{tabular}{|c|c|c|c|}
\hline Behavior & Code & Description & Examples \\
\hline $\begin{array}{l}\text { On-Task } \\
\text { Active }\end{array}$ & 'OA & $\begin{array}{l}\text { Making a visible or overt } \\
\text { contribution to the } \\
\text { classroom's learning activity }\end{array}$ & $\begin{array}{l}\text { a) Asking / Initiating / Commenting on class } \\
\text { topics } \\
\text { b) Working on Blackboard / Reading aloud } \\
\text { c) Raising Hand / Smiling or laughing during on } \\
\text { task conversation }\end{array}$ \\
\hline $\begin{array}{r}\text { On-Task } \\
\text { Passive }\end{array}$ & 'OP' & $\begin{array}{l}\text { Working privately or non- } \\
\text { overt on-task behaviors }\end{array}$ & $\begin{array}{l}\text { a) Taking notes and / or reading class } \\
\text { textbooks or working on assigned class } \\
\text { activity } \\
\text { b) Looking at teacher (or person speaking and } \\
\text { / or working on class related topic) / Working } \\
\text { on computer }\end{array}$ \\
\hline $\begin{array}{l}\text { Off- } \\
\text { Task } \\
\text { Active }\end{array}$ & 'XA & Disruption to on-task activity & $\begin{array}{l}\text { a) Interfering with others' on-task work / } \\
\text { Making remarks unrelated to class topic } \\
\text { b) Smiling or laughing in response to off-task } \\
\text { conversation }\end{array}$ \\
\hline
\end{tabular}


Off- 'XP' Working and other nonsocial Task activities unrelated to class. a) Reading material or taking notes on material unrelated to class topic

b) Looking away from teacher (or person speaking on class related topic) / Looking at peer speaking or working on something that is off task

Table 3. Social partner behavior codes

\begin{tabular}{ccll}
\hline Behavior & Cod & \multicolumn{1}{c}{ Description } & \multicolumn{1}{c}{ Examples } \\
\hline Approval & 'A' & $\begin{array}{l}\text { A display of direct } \\
\text { affirmation of a focal child's } \\
\text { behavior (usually } \\
\text { accompanied by emotion). }\end{array}$ & a) Praising (e.g. 'That's great!') \\
Cooperate & 'C' & $\begin{array}{l}\text { A display of indirect } \\
\text { approval to target child's } \\
\text { behavior. }\end{array}$ & $\begin{array}{l}\text { a) Complying with a request /imitating a } \\
\text { social partner's behavior }\end{array}$ \\
$\begin{array}{c}\text { Factual } \\
\text { Disagreem } \\
\text { ent }\end{array}$ & DIS & $\begin{array}{l}\text { A difference of opinion of } \\
\text { focal child (same topic, } \\
\text { different ideas) }\end{array}$ & a) Stating a fact / cooperative correction \\
Disapprov \\
al
\end{tabular}

The focal child's behavioral codes were based on conceptualizations of classroom 
engagement as a key marker of children's emotional and behavioral engagement and academic motivation (e.g., Skinner, Kindermann, \& Furrer, 2008). The social partner codes, were adapted from Charlesworth and Hartup (1967), Horn, Conners, and Wells (1986), and Kerr, Zigmond, Schaeffer, and Brown (1986), and have previously been used in a number of observational studies to capture classroom social interactions and appropriate (or inappropriate) classroom behaviors (for examples, see Soloman \& Whaler, 1973; Blaze et. al., 2014).

Coding rules. Observations followed the natural sequence of ongoing interactions in the classroom, with two exceptions that applied to situations in which a focal child engaged in the same behavior for a long time (e.g. solitary reading), or when one behavior produced multiple simultaneous consequences (e.g. a joke elicited laughter from many people at the same time). Firstly, any two behavioral codes of children may follow each other in any order, and two consecutive codes of the same kind indicate that the behavior occurred for more than 10 seconds without change. Secondly, a social partner code can only follow individual behaviors, but not other partner codes (so when multiple people laughed, their responses were recoded as if the multiple different partners reacted after each other to the same recoded (joking) behavior of the target individual). In other words, each observation series begins with the behavior of a focal child, and in the case where more than one social partner responded to a focal child's behavior, the individual and partner sequence was repeated.

Observation data-file. The observations are contained in a csv file that I manually re-formatted to a long-formatted structure with complete observations. Long format refers to a longitudinal matrix where each row is one observation (or measurement 
period) and each column represents a variable to classify, or 'identify' the observations (Wickham, 2007, p. 3). The re-formatted observation file contains seven variables: 1) the order in which an observation occurs across the entire file (e.g. overall index value), 2) the day of observation (with 10 distinct values), 3) segment of observation (with 2 distinct values), 4) identity of the focal child (with 23 distinct values), 5) behavior of the focal child (with 4 distinct values) or their social partner (with 6 distinct values), 6) identity of the social partner (with 27 distinct values), and lastly, 7) a description of the social context for interaction exchanges ${ }^{4}$.The following few paragraphs illustrate this structure with a fictitious interaction exchange, as depicted in Table 4 below. This example illustrates the use of coding system to transcribe observe behaviors into a series of events and highlights the use of social partner codes and coding rules.

Table 4. Example long-formatted observational data file

\begin{tabular}{ccccccc}
\hline Index & Day & Segment & Focal & Behavior & Partner & Description \\
\hline 1 & 1 & 1 & Johnny & 'XA' & Johnny & $\begin{array}{l}\text { Johnny makes } \\
\text { joke while } \\
\text { sitting with } \\
\text { Steve and } \\
\text { Larry }\end{array}$ \\
2 & 1 & 1 & Johnny & 'A' & Steve & $\begin{array}{l}\text { Steve and } \\
\text { Larry laugh } \\
\text { simultaneously }\end{array}$ \\
3 & 1 & 1 & Johnny & 'XA' & Johnny & \\
4 & 1 & 1 & Johnny & 'A' & Larry & Laugh \\
5 & 1 & 1 & Johnny & 'XA' & Johnny & $\begin{array}{l}\text { Johnny smiles } \\
\text { at Larry }\end{array}$
\end{tabular}

\footnotetext{
${ }^{4}$ Note, that the four values of focal child's codes correspond to the four behavioral categories and the six values of social partner codes correspond to the six behavioral categories (shown in Tables 1 and 2, respectively). The identity of the Social partner has twenty-seven values, including all twenty-five children, the teacher, and any additional adult. For readers familiar with $\mathrm{R}$, these values are 'levels', of each variable (as converted to a factor variable type) (R Core Team, 2016)
} 


\begin{tabular}{|c|c|c|c|c|c|c|}
\hline 6 & 1 & 1 & Johnny & 'OP' & Johnny & $\begin{array}{l}\text { Johnny starts } \\
\text { writing notes } \\
\text { (over 10 } \\
\text { seconds) }\end{array}$ \\
\hline 7 & 1 & 1 & Johnny & 'OP' & Johnny & Writing \\
\hline 8 & 1 & 1 & Johnny & 'OA' & Johnny & $\begin{array}{l}\text { Johnny raises } \\
\text { hand and } \\
\text { Larry whispers } \\
\text { to Johnny }\end{array}$ \\
\hline 9 & 1 & 1 & Johnny & 'XA' & Johnny & $\begin{array}{l}\text { Johnny } \\
\text { jokingly } \\
\text { pushes Larry } \\
\text { away }\end{array}$ \\
\hline 10 & 1 & 1 & Johnny & 'D' & Larry & $\begin{array}{l}\text { Larry says } \\
\text { stop and calls } \\
\text { Johnny a dork }\end{array}$ \\
\hline
\end{tabular}

In this example, Johnny (the focal child) is sitting at a table with two peers (e.g., Larry and Steve). Note, that Johnny is the focal person for this entire 10-event exchange, but that observations 2,4 , and 6 contain social partner codes. In the current study, social partner codes represent the sample space of potential reinforcers, and focal children's behaviors, represent the sample space of all potentially reinforced behaviors.

Observations 1 through 4 show an example of the first re-coding rule, where two partners provide a consequence simultaneously for the focal child's behavior. Notice that this results in the behavior appearing twice in the data-file - while it only occurred once in the observations. Observations 6 through 7 demonstrate the second coding rule; where the focal child was writing notes for over 10 seconds. By this rule, the observed behavior appears twice (as a repeated code) in the data-file, simply because it occurred continuously for over 10 seconds. 


\section{Chapter 5}

\section{Methods 2: Describing Reinforcement in the Stream of Observations}

This method's chapter formally introduces the terminology, notational conventions, and general concepts that I use in the remainder of this thesis to describe the detection of reinforcement patterns along a stream of observations. I describe these concepts at an overall level of analysis, where reinforcement is presented as a process detectable on an overall series across all individuals, as if all observations were made on a single person (as an aggregated observation series). I formally introduce a recounting procedure used to restructure naturalistic observations so that behavior proportions can be compared 'before' and 'after' each reinforcer as it occurs in its natural order in the observation series.

The methods in this thesis draw inspiration from two sources: Skinner's (1938) tradition of behaviorism for defining reinforcers, and Bakeman and Gottman's (1996) lag-sequential analysis for naturalistic observations. To date, these two traditions have not been synthesized along naturalistic observations, mainly, due to the 'messy' structure of naturalistic observations. Unlike experimental designs, naturalistic observations lack control over delivery of reinforcers on participants behaviors. Consequently, comparisons are not balanced across subjects, and thus, some participating individuals may not receive any reinforcers, while others, may receive multiple reinforcers. Additionally, techniques from lag-sequential analysis are limited in that they are best used for small 'lags' (e.g. immediate behavior-consequence relations) and test the dependent variable in one 
direction at a time (e.g., proportion of target behaviors as a consequence, given a target antecedent).

The recounting procedure introduced in this study attempts to blend these two traditions by allowing any set of observations to be classified into a 'before' or 'after' reinforcement group- When applied to a set of observations, the recounting procedure produces a 'recounted data-file', which contains variables used to indicate and classify observations as occuring before or after reinforcers, along a 'recounted observation series'. This datafile provides a basis for a regression based primary analysis, such as general linear models for binary outcomes (see chapter 6 , for details on the implementation of these methods in this thesis), or a contingency table analysis that follows more from the tradition of contingency detection strategies, such as lagsequential analyses (e.g. Bakeman and Quera, 1995).

This chapter introduces the recounting procedure, using the terminology and concepts used throughout this thesis. I focus on the classification piece of the recounting procedure, specifically, on what it means to classify observations using an 'overall strategy'. This section also discusses the contingency table representation used mainly in the secondary analysis to summarize the counts of observations falling into the categories of the dependent and independent variables. Finally, I discuss the details of both analysis strategies. This includes a specification of the general estimating equation models for the primary analysis as well as three different contingency table representations of the recounted series, and how they may provide slight differences in the $z$-test used to test the secondary hypotheses.

\section{The Recounting Procedure}


I describe the recounting procedure with the aid of Figure 1 (reprinted as Figure 6) to provide a visual and conceptual reference for the terminology I use to refer to this algorithm throughout the remainder of this thesis. I divide this reprint into two components: a simple example observation series as it may appear from a raw set of observations, and then the same series with additional features introduced by the recounting procedure to aid in the detection of reinforcers. Figure 5 shows the sample of observations and Figure 6 shows the line in addition to the recounting procedure used to iteratively measure behavior change as reinforcement occurs, where the rectangles represent the moving probability windows. I first introduce the sample of observations (and relevant terminology and concepts) and then the process of recounting the observations in order to detect putative reinforcers, accordingly. I end this section with a summary of the procedure with an additional visual aid of the algorithmic process.

Figure 5. Example classroom observation series

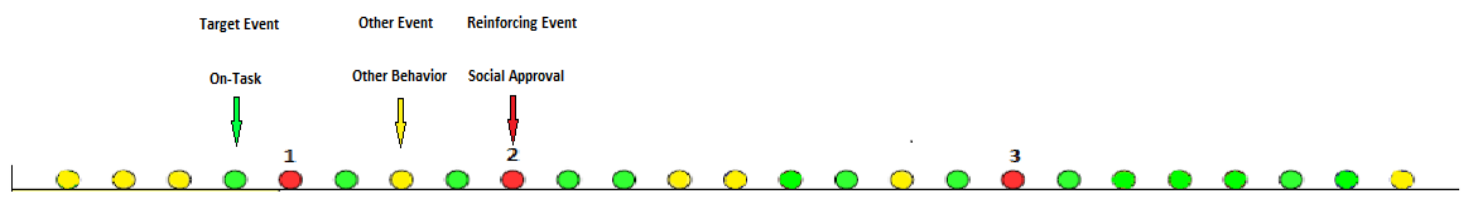

Series and Events. Figure 5 depicts a stream of observations, using the behavior codes tested under Hypothesis 1 (see Chapter 3), that will serve as a sample for the detection of putative reinforcers. I use the term series to denote the sample of observations used to detect putative reinforcers. The term overall series refers to a series that is to be analyzed across all variables for which observations are repeatedly measured (e.g., focal child ID, day of observations, etc.). In this way, the term overall series simply 
refers to a set of observations unfolding across time; Because this thesis exclusively examines observable behavioral events, such as On and Off-Task classroom behaviors, for example. I use the terms behaviors, observations, and events interchangeably when discussing the dataset and derived examples used in this thesis.

Dependent and independent variables. The observations of a given series form the basis of the dependent and independent variables in a reinforcer analysis. As the dependent variable, observations can assume one of two values; targets and non-targets. The recounting procedure 'recodes' these variables using a binary coding scheme, where target observations are recoded as ' 1 ' and non-targets as ' 0 '. In this example, all green circles represent the target 'On-Task' behaviors, while yellow circles represent all other values, for eligible observations of the dependent variable.

The term reinforcement sequence refers to the independent variable in the reinforcer analysis. As an independent variable, the reinforcement sequence has two categories; 'before' or 'after' reinforcers. In terms of Skinner's (1953) definition of reinforcers as consequences which "... increases the probability of further responding", the reinforcement sequence can classify observations according to 'past' and 'future' responding, relative to reinforcers, all within the single overall observation series. To properly classify observations in this way, the intervals corresponding to each category of the reinforcement sequence requires knowing the location of each reinforcer in the series (via the 'overall index') as well as the number of total reinforcers.

All observations leading up to the reinforcer's index are classified as 'before reinforcement' and all the observations following the reinforcer's index are classified as 'after' reinforcers. To compare 'past' and 'future' observations, we simply compare 
proportions across these two reinforcement sequences.

Overall application of the recounting procedure. The 'overall strategy' refers to the application of the recounting procedure across persons, observation episodes (or days), schools, elections, or any other variable in a dataset and that can be used to cluster observations of the dependent variable. In terms of sampling theory, the overall strategy treats observations as 'cluster samples', that is, each observation is assumed to be coming from only one 'cluster'. The term cluster refers to a sampling group in which observations share some common feature (e.g., belonging to same focal person) (Kish, 1998,p. 6). The Sage and Kindermann (1996) observations clearly violate this assumption by containing multiple observations across multiple individuals. This issue be resolved in the primary analysis which accounts for the effects of larger clusters. Generally, it is assumed that repeated measures occur within one cluster.

Figure 6. Recounting procedure applied to classroom observations to detect reinforcers.

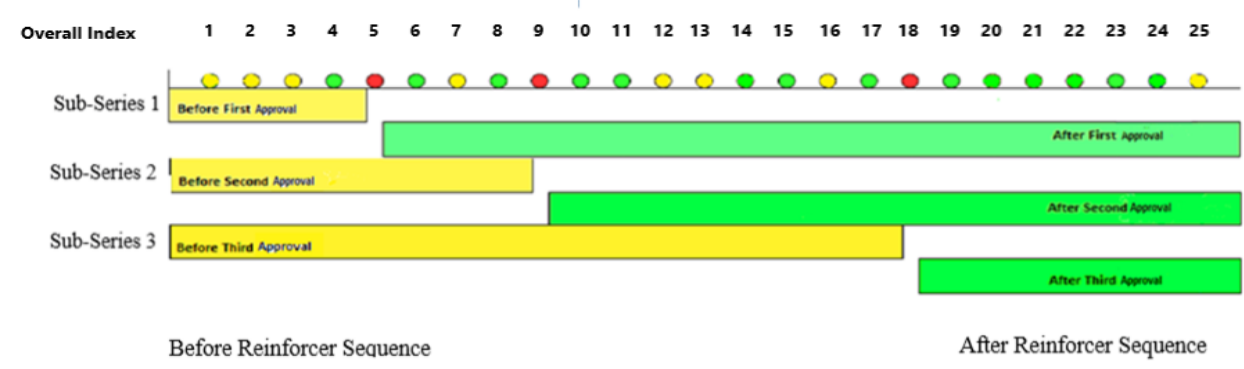

Figure 6 (see above) depicts the application of the recounting procedure using an 'overall strategy' on an observation series. As time goes onwards, the proportion of green 
circles increases in the figure. Using Skinner's (1953) definition of reinforcers, this pattern suggests a reinforcing effect with respect to some other 'observed event'. The three 'sub-series' depict each time the observations are classified by reinforcement sequence. The sub-series depend on the observation selected as a putative reinforcer - in the case of Figure 6, observations are recounted according to the red circles. By reclassifying observations each time the reinforcer occurs, this procedure solves a common issue in 'real world' observations, namely, where reinforcers can occur at any time and at any rate. Accordingly, this allows observations to be comprehensively classified even when observation occur between reinforcers (before one and after another).

Technical description of the recounting procedure. The goal of the overall strategy and recounting procedure is to simplify a naturalistic observation series such that all events can be categorized as 'before' or 'after' reinforcers, with minimal assumptions and demands on the data (i.e., sample size of reinforcers, target behaviors, etc.). Unlike experimental studies, naturalistic observations may capture reinforcement in its naturalistic state; where there is no apriori pattern known to researchers, that connects observed behavioral patterns to reinforcers (e.g., reinforcement schedules, single trials, etc.). The ultimate goal of the recounting procedure is to accurately assess reinforcers when they are not uniformly delivered across individuals for consistent periods of time, with minimal theoretical assumptions. 
Figure 7. Stepwise depiction of the recounting procedure.

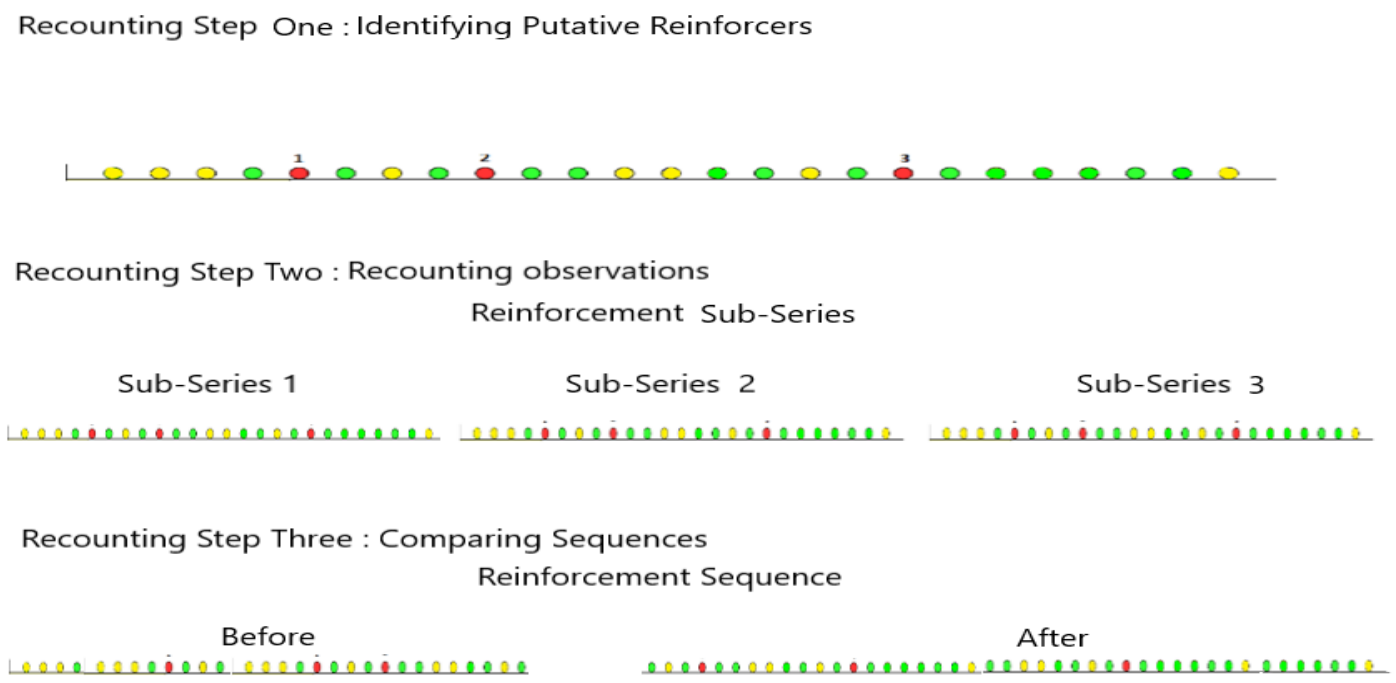

The recounting procedure meets its goals by transforming a dataset so that all the events and a series can resemble a balanced experimental study; where each event falls into a single 'before' or 'after' reinforcement category. There are three steps to this procedure, graphically depicted in Figure 7 . The first step of the procedure is to identify all putative reinforcers on the overall series, without looking at any other variables except the observations. This is necessary for determining the number of $M$ of sub-series, or $M$ replications of the original series, where $M$ is the total number of reinforcers. The second step is to label each replication by its respective sub-series (e.g., sub-series 1, 2, etc.). As described in more details in the next few paragraphs, this thesis implements the recounting procedure in R Shiny (Chang et al., 2016), which produces a dummy column variable for sub-series, that repeats the sub-series ID (i.e. 'sub-series 1', 'sub-series 2', etc.) across all recounted events. The final step is to assign all concatenated recounted 
observations into a 'before' or 'after' reinforcement sequence. While each recounted observation is technically associated with a sub-series, the figure illustrates this last step overall sub-series, so that any statistical comparison is made on the fully classified series of recounted observations.

Contrasting the recounted data file with the original observation file. This thesis implements the recounting procedure in a suite of functions that are available in an $\mathrm{R}$ program that is distributed as a Shiny web-app (described in more detail in Chapter 6). The recounting function takes an observation series as the argument input and produces a spreadsheet-like data frame that is also exported as a csv file. This 'recounted data-file' preserves the variables and structure of the original datafile, but differs in three important ways. The first major difference is that the recounted file contains a 'recounted series' of length $N x M$-- where $N$ is the number of events in the original sample (indexed by $i$ ) and $M$ is the number of reinforcers, or sub-series (indexed by $t$ ). To keep track of the recounted observations, a recounted index is also added in the recounted data-file, in addition to the original and sub-series indices.

A second difference between the recounted and original data-files is the addition of the sub-series and reinforcement sequence variables. The numeric variable sub-series corresponds to the $t^{\text {th }}$ re-counted set of observations (i.e. $t^{\text {th }} s u b$-series) from the $t^{\text {th }}$ iteration of the recounting procedure. The categorical variable reinforcement sequence corresponds to a given whether the event came before or after reinforcer $t$ (for sub-series t). For flexibility in statistical analyses, the reinforcement sequence is dummy coded so that a code of 0 indicates a given observation corresponds to the before-reinforcement sequence and the code 0 indicates the observation falls in the after-reinforcement 
sequence.

A final difference between the two files is in the recoded observation variable that is created for the recounted data-file. As the dependent variable, the recoded event is a numeric variable using a 0 for non-target observations and a 1 for target observations. Note, that since reinforcers divide each sub-series into reinforcement sequences, the reinforcer corresponding to each sub-series is removed from that particular sub-series.

For all other sub-series, previous and future reinforcers are coded as 0 , since they are non-target observations. Table 5 (see next page) illustrates the transformation of an original series into the recounted series. This table is based off the observations in Figure 7 (a truncated example from Figure 1), where the 'Focal Child ID' is arbitrary for this example.

Figure 8. Shortened example data series (conceptual)

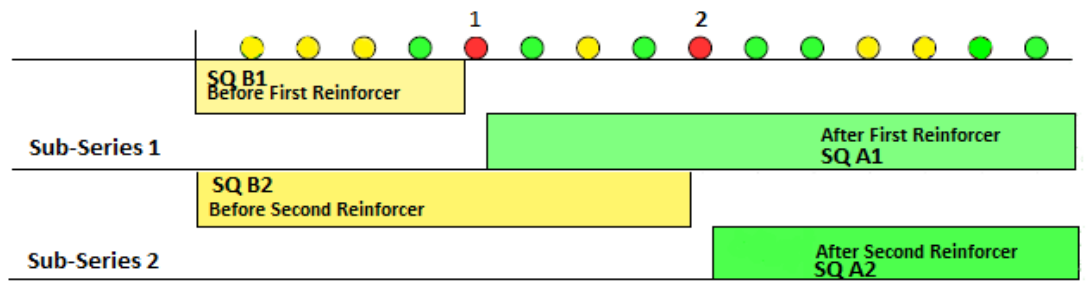

Table 5. Shortened example recounted data series (data-frame)

\begin{tabular}{|c|c|c|c|c|c|c|c|c|c|}
\hline \multicolumn{3}{|c|}{ Original Data-File } & \multicolumn{7}{|c|}{ Re-Counted Data-File } \\
\hline Focal & Index & Obs. & $\mathrm{F}$ & Overall & Recounted & Obs & Re-coded & Sequence & Sub- \\
\hline$(\mathrm{F})$ & (I) & & & (I) & (I) & . & Obs. & & series \\
\hline Abe & 1 & $\mathrm{Y}$ & Abe & 1 & 1 & 0 & 0 & 0 & 1 \\
\hline Abe & 2 & Y & Abe & 2 & 2 & 0 & 0 & 0 & 1 \\
\hline Abe & 3 & Y & Abe & 3 & 3 & 0 & 0 & 0 & 1 \\
\hline
\end{tabular}




\begin{tabular}{|c|c|c|c|c|c|c|c|c|c|}
\hline Abe & 4 & G & Abe & 4 & 4 & 1 & 0 & 0 & 1 \\
\hline Abe & 5 & $\mathrm{R}$ & Abe & 5 & 5 & 0 & 1 & 1 & 1 \\
\hline Abe & 6 & G & Abe & 6 & 6 & 1 & 1 & 1 & 1 \\
\hline . & . & . & . & . & & . & . & . & . \\
\hline . & . & . & Abe & 14 & 14 & 1 & 1 & 1 & 1 \\
\hline . & . & . & Abe & 15 & 15 & 1 & 1 & 1 & 1 \\
\hline Abe & 14 & 1 & Abe & 1 & 16 & 0 & 0 & 0 & 2 \\
\hline Abe & 15 & 1 & Abe & 2 & 17 & 0 & 0 & 0 & 2 \\
\hline \multirow{6}{*}{\multicolumn{3}{|c|}{ End Of Observation }} & Abe & 3 & 18 & 0 & 0 & & 2 \\
\hline & & & Abe & 4 & 19 & 1 & 0 & 0 & 2 \\
\hline & & & Abe & 5 & 20 & 0 & 0 & 0 & 2 \\
\hline & & & Abe & 6 & 21 & 1 & 0 & 0 & 2 \\
\hline & & & . & . & & $\cdot$ & . & . & \\
\hline & & & Abe & 15 & 30 & 1 & 0 & 0 & 2 \\
\hline
\end{tabular}

\section{The Overall Strategy and its Representation in Contingency Tables}

The recounted data-file provides the necessary components for the primary and secondary analysis strategies used to adress the research questions in this thesis. This section continues on the topic of contingency detection versus reinforcement detection (see Chapter 3); and focusing on construction of the contingency tables used in the secondary analyses. Naturally, this begins with a typical approach to representing observations 'before' and 'after' some events for clustered samples. This leads to a discussion as to why primary analysis is not replicated on contingency tables, and is left as a future direction for future studies. 
This discussion leads into details regarding the tables for a lag-sequential analysis, and emphasizes the conceptualization of the recounting procedure as an extension of this strategy. I conclude this discussion by showing how concepts from either approach (e.g., matched pairs or lag-contingencies) can be adapted to the recounting procedure to produce 'before' and 'after' reinforcer contingency tables from a transition table of event transitions.

Measuring the dependent variable across the recounted series. Recall that the overall strategy treats the recounted series as belonging to one 'focal person'. Figure 9 shows such an example series where there are 3 events before and 3 after the red circle (i.e., reinforcer). The events are labelled in three ways. On top of the circles is the overall index value which counts from the first observation to the last event in the series. Beneath the circle is a second index for matched pairs. This index was not introduced earlier since it is not explicitly used in the recounted procedure. I introduce it now to illustrate an equivalence in the recounting procedure and a modified version of a traditional approach for 'before' and 'after' event comparisons for matched pairs. As this traditional approach uses assumptions met during more controlled observations (e.g., experimental), this section focuses on some necessary adjustments to concepturally envision reinforcement detection in naturalistic observations from matched pairs.

Contingency tables for matched pairs. Agresti (2013) uses to term 'matched pairs' in reference to dependent data structures in the context of count based outcomes, where matched pairs are defined as '[datasets]... when each observation in one sample pairs with an observation in the other sample' (pp. 413). Matched pairs analyses assume that each record contains a measure of the dependent variable along two occasions and that 
each occasion is related in some way (e.g., belong to the same cluster). This assumption is convenient for studies looking at changes in voting behaviors, for example, where the only data of true interest is where a study subject submits their vote at both time points. Under these conditions, contingency table tests are available for complete matched pairs, where the hypothesis is testing whether proportions of voting behaviors are the same across occasions (e.g., 'before' and 'after' some event).

Figure 9. Illustrative example of overall matched pairs

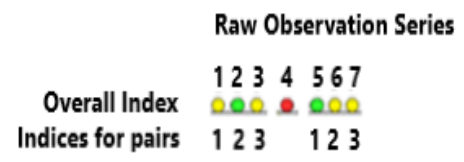

Figure 9 shows that each observation 'before reinforcement' contains a corresponding observation 'after reinforcement', where the 'reinforcer' is depicted by the red circle. In this case, the pairings are related only by their order in respect to reinforcers; beginning with the first observation and 'resetting' as the reinforcer occurs (e.g., first observation paired with first observation after reinforcer, second with second, etc.).While Agresti's definition implies that the 'matching' is linked to some higher clustering variable (e.g., focal person ID, day of observations, etc.), I ignore this for now, and focus on just the pairings themselves, created from their relative order to reinforcers, as this is most easily viewed through the lens of an overall strategy.

Figure 10. Data structure and transition table for matched pairs 

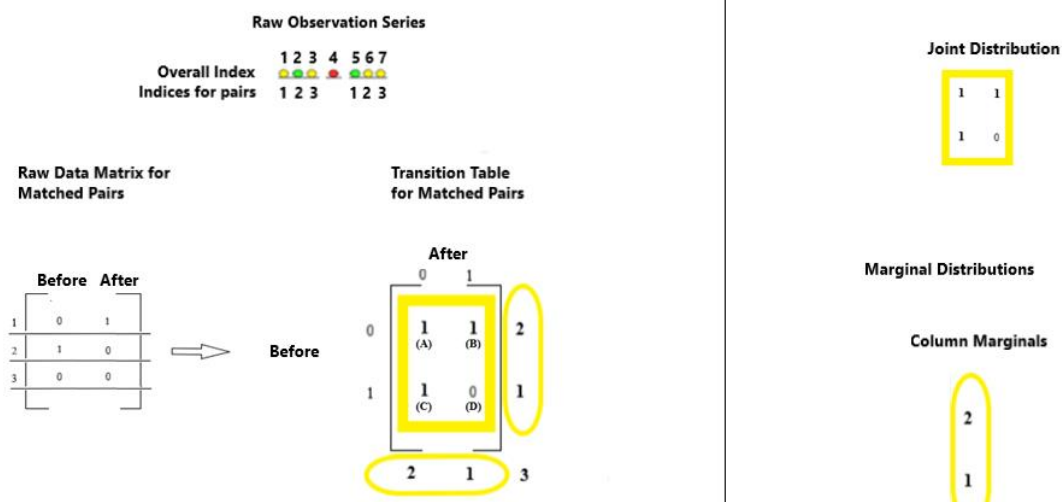

Marginal Distribution

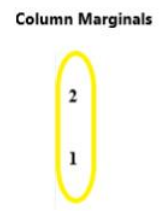

Row Marginals

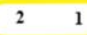

Figure 10 shows the construction of a 'contingency table" for the transitions in the dependent variable across time for all matched pairs. Agresti (2013) formally defines a contingency table as a 'rectangular table having $I$ rows for categories of $\mathrm{X}$ and $J$ columns for categories of Y [that] displays the $I J$ possible combinations of outcomes' (pp. 37). The figure shows the construction of such a table from the raw observations. This contingency table is known as a 'square contingency table' or a 'transition table ${ }^{6}$, in the lag-sequential literature, and is defined as a table where the same variable populates the row and columns of the two-way contingency table" (Agresti, 2013, pp. 413). The hilighted areas of these tables show two distributions used to peform the secondary analyses tests in this thesis. These 'joint' and 'marginal' distributions will come up throughout the remainder of this thesis when discussing the statistical tests for the secondary analyses.

${ }^{5}$ Gottman \& Roy $(1990$, p.222) note that the term contingency table should be reserved for a crossclassification of independent cells. In this thesis, I use Agresti's definition for all rectangular displays of tabulated observations.

${ }^{6}$ This thesis uses the term 'transition table' in the tradition of a lag-sequential analysis. 
Figure 11. Contingency table for marginal distribution of matched pairs.
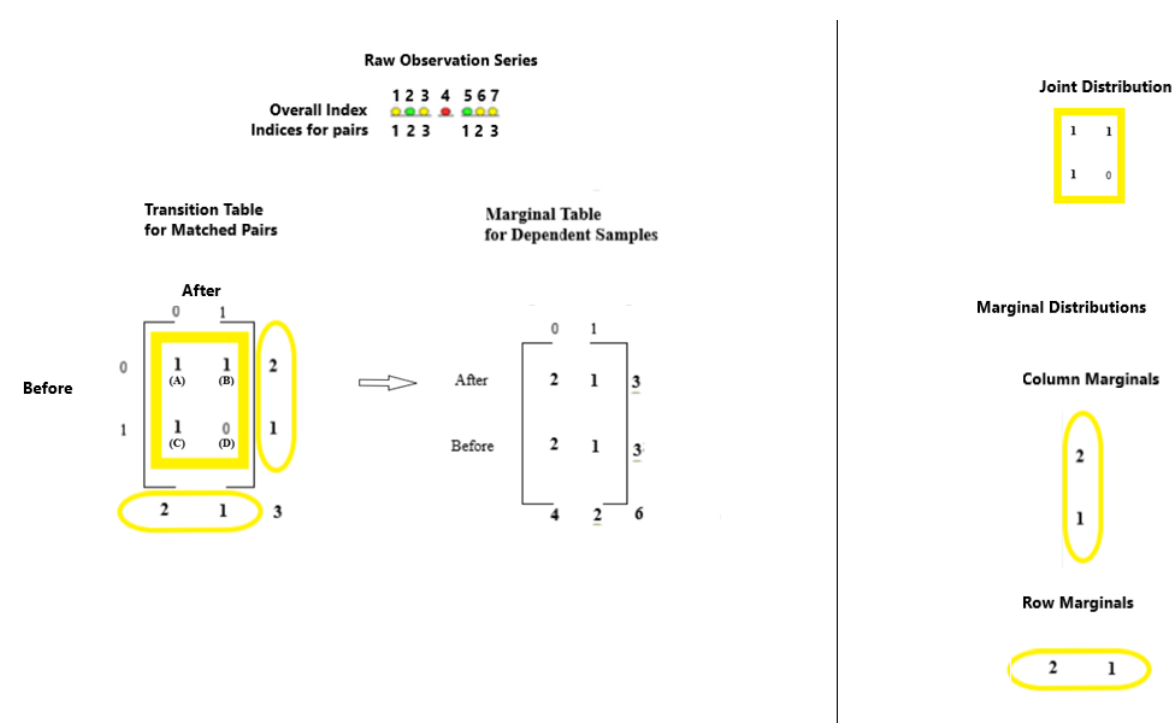

For paired observations, the cells of the 'transition table' (as it called for the remainder of this thesis), form the joint distribution or the total observations of a given pair of values of the dependent variable at the first and second time points. The outer borders of the table show the marginal distributions; that is, the total number of observations of each category of the dependent variable before reinforcers (see the row margins), and the total number after reinforcers (see the column margins). As Figure 11 shows, the marginal distribution can be represented in a 2 × 2 contingency table which shows the total observations for each combination of categories, across all pairs.

\section{Equation 2. Mcnemar test statistic}

$$
\mathrm{X}^{2}=\frac{(B-C)^{2}}{(B+C)}
$$

To test the hypotheses that the proportions of transitions from 'no' to 'yes' differ from 'yes' to 'no', we would enter the values of the joint distribution into the Mcnemar test equation (shown in Equation 2). This is called a test for marginal homogeneity 
(Agresti, 2013, pp. 414). and can determine whether there is a shift in the proportions across time. The reader may easily see this as a strategy for testing the primary hypotheses that the proportion of target behaviors is greater following reinforcers compared to before reinforcers. In addition to reasons discussed in the following paragraphs, this thesis does not use such a strategy for the secondary analyses.

Alternatively, the secondary analyses test proportions indirectly, as an extension of the lag-analysis tests to reinforcers.

Figure 12. Data structure and transition table for matched pairs
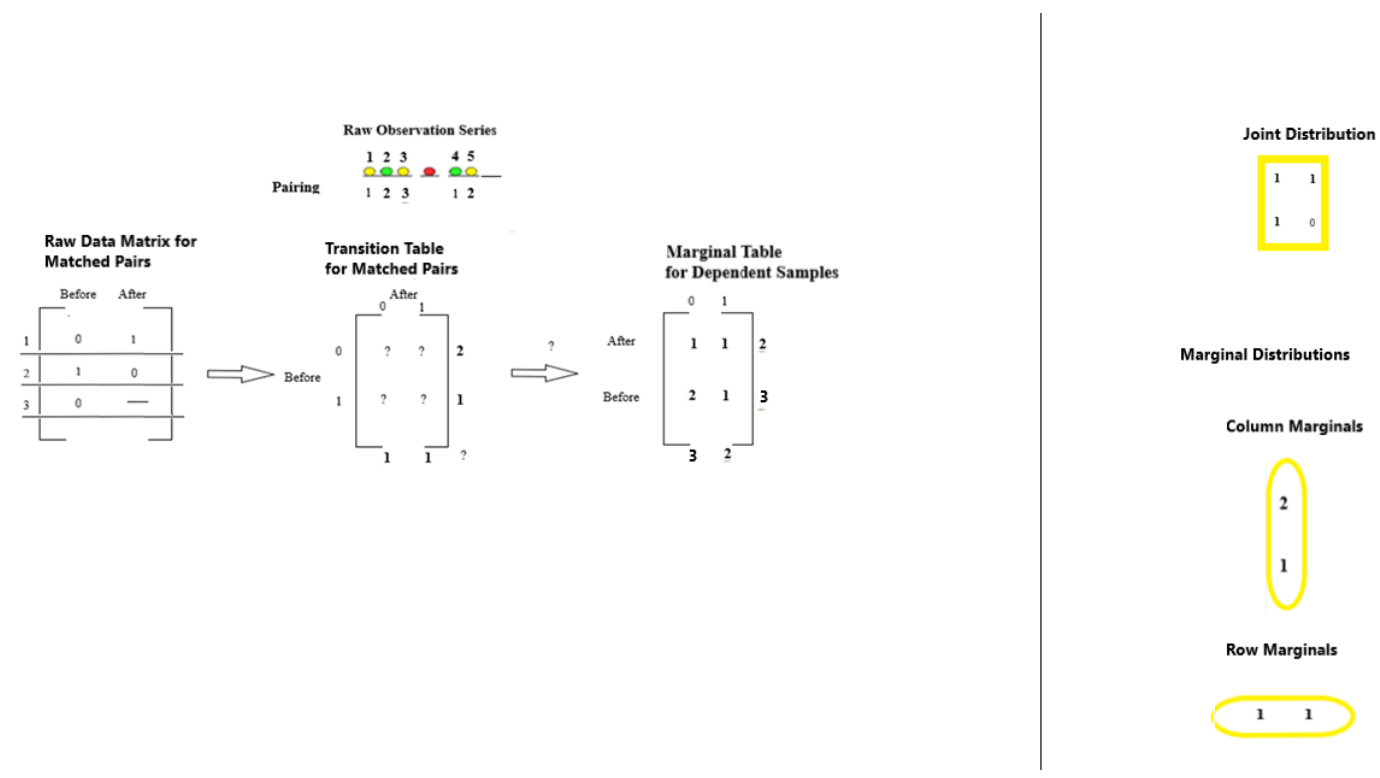

Non-matched pairs. For datasets meeting the matched pairs assumption (e.g. complete records at both time points, for each case of pairs), the marginal tables are directly obtained from the $2 \times 2$ transitional table. Figure 12 shows a scenario for which the assumption of complete matched pairs does not hold true for the third case. In this scenario, the margins of the transition table show the complete distributions based on the Figure, and are not computed from the joint distribution of the complete pairs. To 
compute a Mcnemar test on this set of observations, we must either constrict the sample to complete pairs or use an alternative strategy for incomplete pairs.

Figure 13. Contingency table for marginal distribution of matched pairs.
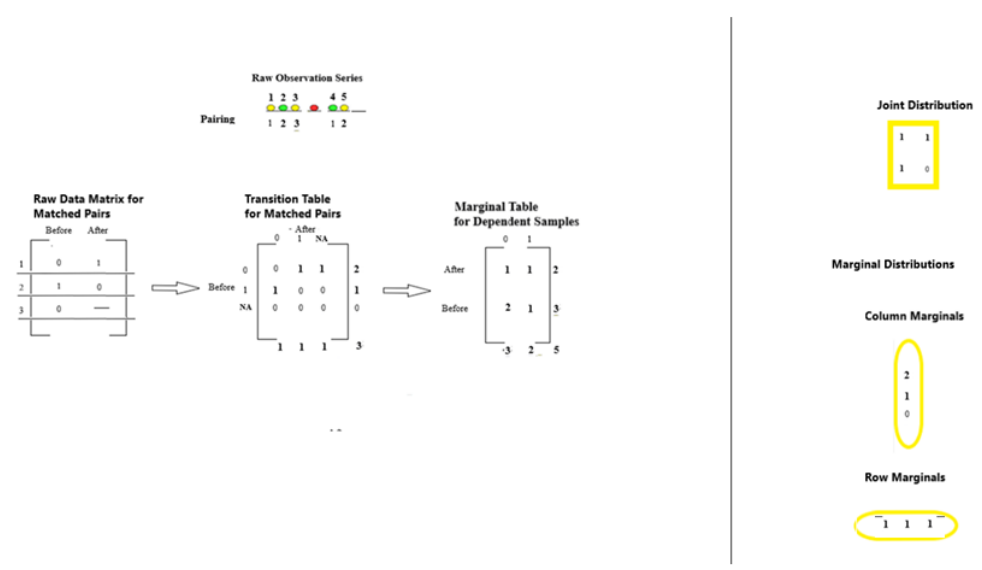

Figure 13 shows one way of computing the transitional and marginal tables for incomplete pairs. This table applies the same logic to obtain the marginal values from cell transitions, but expands the square table to a $3 \times 3$ table so that it includes a value for missing observations (denoted by $N A$ ) in each category of the dependent variable (for each category of the independent variable). As the next section shows, this strategy can also be applied to the tables produced by the recounting procedures, in order to obtain a contingency table for the recounted frequencies directly from the transitions of incomplete pairs (in addition to the original conceptualization as the sum of sub-series tables).

This presence of incomplete pairs presents a dilemma for a traditional Mcnemar 
use of the Mcnemar test and thus leaves researchers with the option to either discard incomplete pairs or use one of many special variations of the Mcnemar test for 'messy' data (see Choi \& Stablein, 1982 ; Zhang, Cao, \& Ahn, 2017). In line with the original scope of this thesis, I do not use either of these strategies, and instead apply a traditional contingency table test used in the contingency analyses literature, which relaxes some of the data requirements of a Mcnemar test (e.g., square transition tables, complete pairs), and aligns with the original conceptualization of the reinforcement detection procedure as an extension of lag-analysis in both directions, across all observations.

Allison and Liker z-score on the marginal contingency table. The Allison and Liker $z$-score has a long history in testing reinforcement hypotheses from naturalistic observations (e.g. Gottman and Roy, 1990). In its original usage, this test compares the proportions of target behaviors occuring immediately after some 'event' to the overall base-rate probability of the target behaviors, across the entire series of events. In terms of lag analysis, the target behavior is termed a consequence and time $t+1$ while the 'event' is the antecedent at time $t$. The lag-analysis uses a transition table to count all pairs of observations in an overall series, where pairs are observations of the form $\mathrm{Y}_{\mathrm{t}+1}, \mathrm{X}_{\mathrm{t}}$. As explained in the following paragraphs, this is conceptually like a reinforcer analysis, with a lag extended across all observations and in both directions (i.e., dependent variable lag forward and dependent variable lags behind the independent variable). 
Figure 14. 3 X 3 transition table for lag-one pairs.

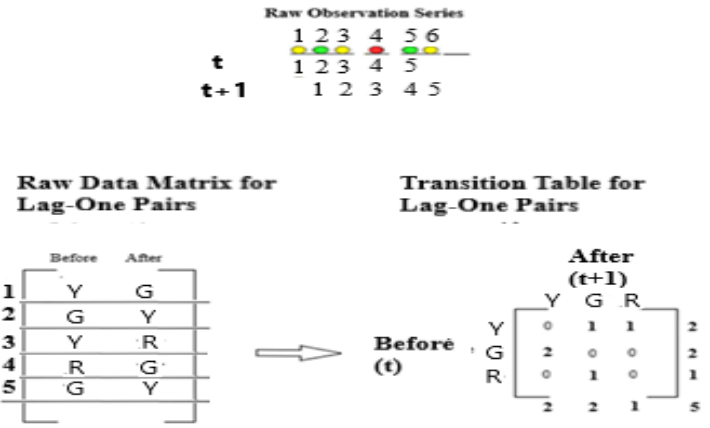

Figure 14 shows a transition table to test one of Sage and Kindermann's (1999) hypothesis that Social Approval (depicted by red circles) is more likely to follow OnTask behaviors (depicted by Green circles). The top of this figure shows the indices used to match consecutive observations to form pairs by their natural ordering. This does not account for any 'higher order' clustering (e.g., same individual subjects), and simply uses the ordinary index as a bases for matching observations at time $t$ with time $t+1$. The 'Transition Table for Lag-One Pairs' shows the full tabulation of these pairs. This is analogous to the $2 \times 2$ 'square' table for paired transitions used in a Mcnemar analysis, except it includes all observations (versus just the binary dependent variable). This opens a traditional lag-sequential analysis to testing hypotheses regarding consequences as well as antecedents under naturally occuring observations. 
Figure 15. 2 X 2 marginal table for directed lag-one hypothesis.

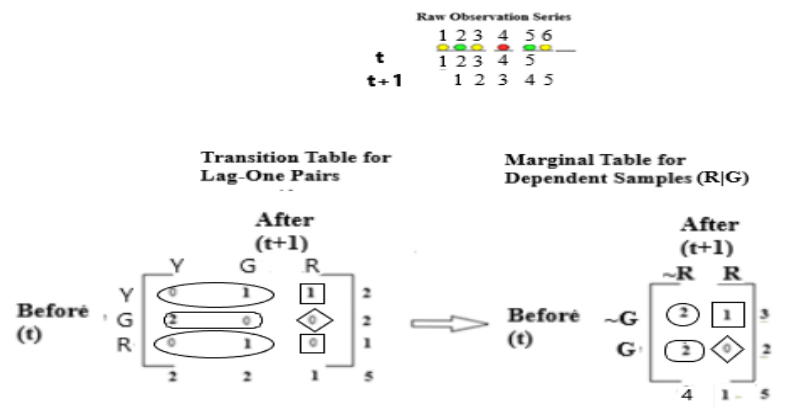

To test Sage and Kindermann's (1999) hypothesis that Social Approval (e.g. red circles) are more likely to follow On-Task behaviors (e.g. green circles) than all other antecedents, we need to know; a) the distribution of all antecedents, b) the distribution of On-Task antecedents, and c) the distribution of Social Approval consequences. Figure 15 shows the construction of a 'Marginal Table for Dependent Samples' in the context of a lag-sequential analysis for the stated hypothesis. This table differs from the marginal table for matched pairs (See Figure 13) since it does not consider the sums across the full margins of the transition table. This is because the antecedent is paired with a different 'target' (e.g. green circles) than the target consequence (e.g. red target). Accordingly, the 'non-target' cells for the antecedent in the marginal table sum across the ' $\mathrm{Y}$ ' and ' $\mathrm{R}$ ' antecedent codes, while the 'non-target' cells for the consequence sum across the ' $\mathrm{Y}$ ' and 'G' consequence codes.

Construction from matched pairs. The reader may notice that the 'marginal table' for the lag-one consequence cannot answer whether a target behavior is more likely to follow a behavior-before reinforcers or after-reinforcers. This is largely a result of the traditional lag-sequential hypothesis; which examines either the consequence given the 
antecedent or the antecedent given the consequence (The interested reader is directed to Appendix $\mathrm{G}$ for a table showing the construction of marginal table for a lag-one, 'before' (e.g. lag-behind) and 'after' (lag-forward), sequential analysis). I presented this table was to draw the connection between a set-up for a lag-sequential analysis, based on marginalizing across the relevant values of a $3 \times 3$ transitional table, and the set up used in a reinforcer analysis. Conceptually, the reinforcer analysis extends the notion of a contingency analysis to all antecedents and all consequences to examine large windows of behaviors rather than the immediate lags.

Figure 16. Equivalency of marginal table of incomplete pairs and recounting procedure

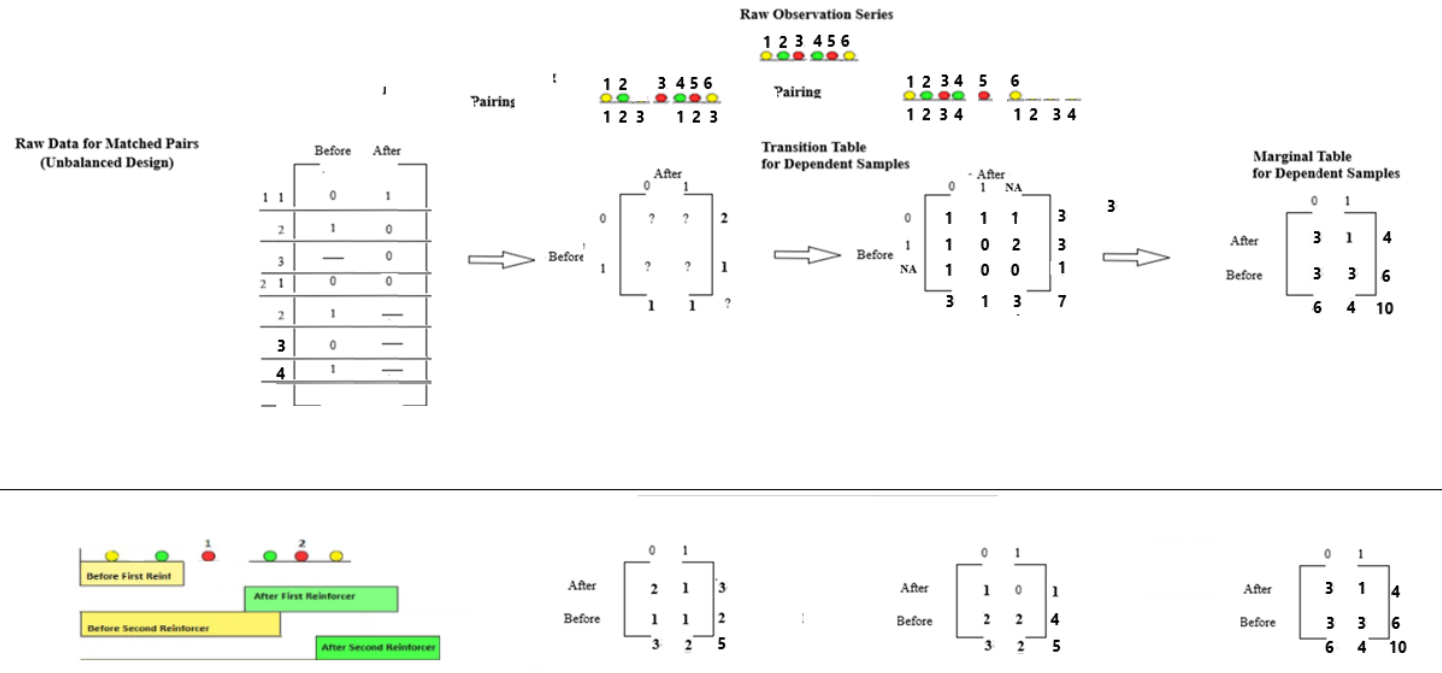

Figure 16 shows an observation series with two reinforcers to demonstrate the construction of a 'Marginal Table for Dependent Samples', using two strategies; a transition table for matched pairs (see top portion) and by directly computing probability windows across each 'sub-series', in the spirit of a lag-sequential analysis and then summing across these tables without consideration for matched pairs (see bottom 
portion). As the top portion shows, we can conceive the series in terms of pairing events with their relative index to reinforcers (e.g. before first reinforcer and after first reinforcer, etc.). As the bottom portion shows, we can also cross-classify observations by reinforcement sequence (e.g., 'before' or 'after') for each reinforcer occurance, and then sum these values into one 'Marginal Table for Dependent Samples' (referred to as the 'recounted frequency table', in the remainder of this thesis). As both portions show, the end result is the same, and thus, it logically extends that a test on marginal tables for paired transitions is applicable in either conceptualization of the recounting procedure.

With the equivalence in the construction shown graphically, it is a different process programatically. This thesis originally constructed the recounting procedure with lag-sequential analyses as a reference. In this view, the same hypotheses and same tests can be applied to a reinforcer analysis for sequential dependence, but by extending the 'lag' to all observations and in both directions. This is not to say that a Mcnemar test cannot be used to compare proportions across both sequences. There is a modest body of literature on variants of this test that account for incomplete pairs (e.g., Tang \& Tang, 2003 ; Zhang, Cao, \& Ahn, 2017), and multiple comparisons (e.g., Westfall, Troendle \& Pennello, 2010). Nevertheless, a straightforward application of the Mcnemar test would violate its assumptions, and without an inherent matching of observations (outside the sequential ordering), I leave this test as a future development for the next version of the Reinforcinator application as discussed in Chapter 8, and focus on the Allison and Liker $z$-test for testing sequentially dependent observations, for the secondary analyses in this thesis.

\section{Primary analysis for repeated measures}


The primary analysis compares the proportions of target behaviors 'before' and 'after' reinforcers, sampled across the recounted observation series. This comparison directly follows Skinner's (1938) definitional criteria of reinforcers as “... any consequence of behavior which is rewarding, or more technically, reinforcing, increases the probability of further responding (B. F. Skinner, 1953; p. 345)". Instead of contingency analyses, the primary analyses use a regression-based strategy to directly tests the recounted series, along with indicator variables to indicate the sub-series, reinforcement sequences, and focal individuals (for differentiating observations from different individuals). While the 'overall strategy' classifies observations without regards to separate individuals, it is still of interest to consider the dependency of recounted observations due to occuring to repeated measures of the same individuals. This section discusses the statistical procedure used to account for variation within individuals.

General estimating equations. Repeated observations of the same individuals are more likely to be correlated than observations across different individuals, or generally, across repeated observations of the same 'unit'. Fortunately, general estimating equations (Liang \& Zeger, 1986) extend a regression model to non-linear outcomes (i.e. generalized linear model), while allowing for multiple repeated measures within a 'unit' (e.g. focal child). This class of models accounts for repeated observations by adjusting the standard errors, without explicitly modelling the correlation across within-individual response patterns. In this way, these models can produce the same overall parameter estimates as ordinary regression models while reducing the inflation of standard errors due to repeated measures within individuals.

Generalize Estimated Equations (GEE) models comprise three pieces; a model 
for the mean and variance, and a working correlation matrix for specifying the correlation within observations of the same 'cluster'. As a class of the generalized linear models, GEE's can be used to model non-linear outcomes via a 'link' function for the mean and variance of non-normally distributed outcomes. For binomial outcomes, the logit link is used to model the log odds of the outcome, based on the mean and variance of binomially distributed outcome. In the regression analysis, the overall marginal mean is modelled by this part of the general estimating equations. For the binomial models used in this thesis, all regression coefficients are expressed in terms of 'logits', which can be converted to 'log odds' and then the same proportions identical to those obtained from the marginal table (see Appendix D for these conversion equations).

The third piece of general estimating equations is called the "working correlation structure'. This structure specifies the assumed within-cluster correlation pattern shared across all values of the clusters and is used in an iterative process to calculate the 'robust standard error', which account for the observed within-cluster correlation structures. In this way, the working correlation matrix provides a 'best guess' as a starting value for the within-subject correlation patterns. The actual correlation within subjects is used to update this guess, until the estimated correlation pattern converges to some value (i.e., where consecutive iterations no longer produce significantly different estimates). It is this process that allows gee models to produce consistent estimates of the standard errors even when the working correlation structure is mis-specified (Heagerty \& Zerger, 1996); where consistency refers to a point estimate that best approximates the population value, as the sample size increases (Ramachandran \& Tsokos, 2014, pp.266 ).

There are many variations of the working correlation matrix (see Wang, 2002). 
For unbalanced longitudinal observations, where clusters may have different number of observations, the 'independence' and 'exchangeable' working correlation structures are two common choices. There are advantageous and disadvantageous to each of these structures, especially while considering the goals of the current study. The 'exchangeable' working correlation structure has been shown to be more efficient and theoretically appropriate for longitudinal observations (e.g. Pan \& Connett, 2002). A disadvantage of this structure is that it also affects the interpretation of parameter estimates, since the working correlation structure is used to solve beta coefficients (e.g. Wang, 2014) in addition to optimizing the standard errors. In this way, the parameter estimates under an 'exchangeable' working correlation matrix are not always equivalent to those from an ordinary logistic regression (via a logit link).

All models in this thesis use an 'independence' structure. The independence structure is essentially an identity matrix and does not explicitly state a correlation pattern for within cluster correlations. Consequently, the coefficients using this structure are identical to those obtained from a logistic regression analysis. Because GEE's use an iterative procedure for updating the standard errors based on the observed intra-individual correlation patterns, the standard errors for the coefficients are improved over those from an ordinary logistic regression analysis. For these reasons, all models use the 'independence structure', and the interested reader is encouraged to see Appendix H for the primary analysis results with models using the 'exchangeable' working correlation structures.

\section{Secondary Analysis for difference between reinforced behaviors and base rates along the contingency table}


The secondary analyses use the Allison and Liker $z$-score for sequential dependence to test effects of behavior probability after reinforcers compared to the overall base-rate of 'target' beahviors. As the recounting procedure can be conceptualized as extending a traditional lag-one analysis across all observations, before and after reinforcers, this test follows suite by testing secondary hypotheses inspired by the logic of a contingency test for sequentially dependent observations. This section focuses on the details and assumptions for the construction of these tables via the recounting procedures and does not revist the rationale behind this approach for the secondary analyses.

I use language introduced earlier in this chapter to demonstrate the constrution of the 'recounted frequency table' by way of sub-series contingency tables (versus by incomplete matched pairs). I demonstrate this test statistic on the recounted frequency table and note its limitations. This motivates the derivation of two additional contingency tables, and the demonstration of the test using the same values across these slightly modified versions of the recounted frequency table. 
Figure 17. Stepwise depiction of the recounting procedure (Figure 7 reprint)

Recounting Step One : Identifying Putative Reinforcers

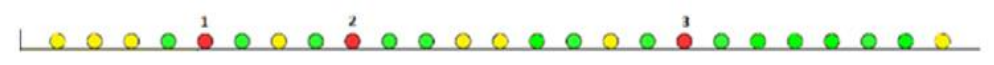

Recounting Step Two : Recounting observations

Reinforcement Sub-Series

$\begin{array}{lll}\text { Sub-Series } 1 & \text { Sub-Series } 2 & \text { Sub-Series } 3\end{array}$

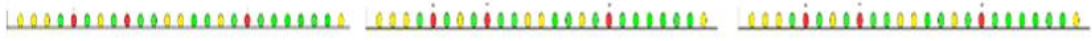

Recounting Step Three : Comparing Sequences

Reinforcement Sequence

\begin{tabular}{|c|c|}
\hline Before & After \\
\hline 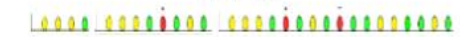 & 000000000000000100000000000000100000 \\
\hline -Series 1 Sub-Series $2 \quad$ Sub-Series 3 & $\begin{array}{lll}\text { Sub-Series } 1 & \text { Sub-Series } 2 & \text { Sub-Series } 3\end{array}$ \\
\hline
\end{tabular}

Derivation and assumptions of the recounted series. I reprint the step-wise recounitng procedure (see Figure 17) to remind the reader that the 'recounted series' results from replicating all observations from the original series by the number of $M$ reinforcrs, where $M$ is the total number of reinforcers (see step two). As step-three figure indicates (see step-three), the recounting procedure classifies events into reinforcement sequences on a sub-series basis. The observations of the concatenated sub-series are then ready for a single contingency table table in which the probability of the dependent variable belonging to a particular category (e.g. target or non-target) can be compared 
across reinforcement sequences.

This approach solves the issues of creating consistent pairings in non-paired observations and allows for an accurate classification of 'messy' observations which fail to meet the assumptions of standard contingency table classifcations (e.g., mutual exclusive membership to a single category). Aditionally, this approach resolves a limitation of lag-sequential analysis from a prespective of reinforcers isnce it accounts for all observations, lagging behind (i.e. 'before' and (i.e. 'after') all reinforcers, at all possible 'lags'. Like a lag-analysis, this strategy also complies well with an overall measure of the dependent variable; so that classifications are made across all individuals.

Naturally, resampling the same observations introduces a dependency in the observations of the recounted series, over and above any other repeated sampling in the original series (e.g. repeated measures of the same individual). This is because each observation in the original series becomes multiple records in the recounted series. While testing each sub-series resolves this issue of resampling, such a procedure fails to give a comprehensive overview of reinforcement since it does not account for an accurate overall classification for events with multiple group membership (e.g., events before and after multiple reinforcers).

While the primary analysis accounts for the dependency in the recounted series by taking advantage of replications nested within sub-series, and sub-series nested within individuals, the secondary analysis relies on the Allison and Liker $z$-test for sequentially dependent observations, without regard to repeated sampling of specific individuals.

\section{Allison and Liker $z$-test on the contingency table for recounted frequencies}

The Allison and Liker $z$-test is a statistic originally used to correct for dependency 
between observations using a lagged analysis. As Gottman and Roy (1990) discuss, the nature of a lag-analysis requires resampling the same events at time $t$ (i.e., the antecedent) and time $\mathrm{t}+\mathrm{l}$ (where $l$ refers to the lag). This test corrects for the dependence introduced when the consequence of one pair serves as the antecedent in the next pair across the full series of observations. In these analyses, the number of pairs used to compute the $z$-score is the total events (i.e. $N$ ) minus the value of the lag. When appropriated to a reinforcer analysis, the correction is similar to a lag-one analysis, and amounts to $N-1^{7}$ for the recounted series; where the reinforcer is subtracted from the total observations in each sub-series.

Figure 18. Reinforcement detection measure (a.k.a. recounting procedure) (Figure 1 reprint)

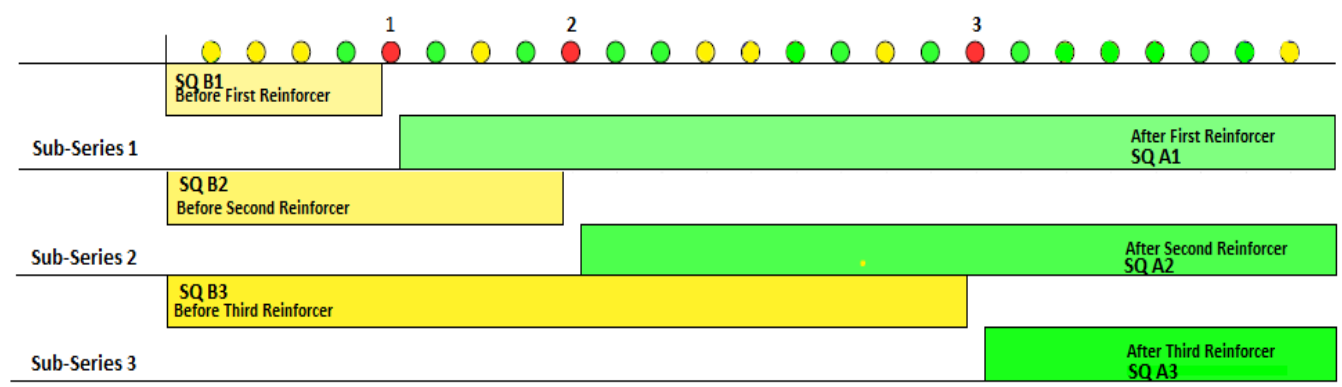

\footnotetext{
${ }^{7}$ The actual correction $(N-1) * M$ but because the reinforcers are subtracted within each sub-series, the correction simplifies to $\mathrm{N}-\mathrm{l}$ for the recounted series and its derived contingency tables.
} 
Table 6. Contingency table for recounted frequencies

\begin{tabular}{|c|c|c|c|}
\hline & \multicolumn{2}{|c|}{ Observation } & \multirow[b]{2}{*}{ Totals } \\
\hline & $\begin{array}{l}\text { Target } \\
\text { (Green) }\end{array}$ & $\begin{array}{c}\text { Other } \\
\text { (Not Green) }\end{array}$ & \\
\hline After & $\begin{array}{c}30 \\
(.7)^{\mathrm{a}}\end{array}$ & $\begin{array}{c}13 \\
(.3)\end{array}$ & $\begin{array}{l}43 \\
\text { (1) [.6] }\end{array}$ \\
\hline Before & $\begin{array}{c}12 \\
(.41)\end{array}$ & $\begin{array}{c}17 \\
(.59)\end{array}$ & $\begin{array}{l}29 \\
(1)\end{array}$ \\
\hline Totals & $\begin{array}{c}42 \\
(.58)\end{array}$ & $\begin{array}{c}30 \\
(.42)\end{array}$ & $\begin{array}{l}72 \\
(1)\end{array}$ \\
\hline
\end{tabular}

a. Values in parenthesis are cell values divided by the row totals (i.e. row-margin proportions). Values in brackets are the base-rate proportions (shown only for target behaviors

Table 6 shows the contingency table for the recounted frequencies, based on the series Figure 18. From this table, we can see the distribution of recounted observations, classified as targets or non-targets, across the reinforcement sequences. This resembles the contingency table shown previously in this chapter for a lag-one analysis (see Figure 15); except the rows contain the reinforcement sequence and the columns represent behaviors, as opposed to the different behaviors as antecedents and consequences. The table shows the recounted frequencies, these are mainly of interest in that they can be used to obtain a variety of proportions, but otherwise, do not provide any real substantive value. The proportions are of most interest since they are preserved by dividing across the sub-series, and thus, reflect the true distribution of events classified into multiple sequences (e.g. before and after reinforcers). 
Equation 3. Allison and Liker z-score for recounted frequencies

$$
\begin{gathered}
z_{1}=\frac{\operatorname{Pr}(Y=\text { Target } \mid x=\text { After })_{\ldots}-\operatorname{Pr}(Y=\text { Target })}{\sqrt{\frac{\operatorname{Pr}(Y=\text { Target })}{(N-1) * P(Y \mid x=\text { After })}}} \\
=\frac{.7-.58}{\sqrt{\frac{.58 *(.42)(.4)}{71 *(.6)}}}=\frac{.7-.58}{\sqrt{\frac{.0974}{42.6}}}=\frac{.12}{.0478} \\
=2.51
\end{gathered}
$$

Equation 3 shows the computation of the Allison and Liker $z$-test for the recounted series. This test compares two proportions; the proportion of target behaviors after all reinforcers and the overall proportion (i.e.'base-rate probability') of target behaviors across all recounted observations. The significant difference in this example would be interpreted as potential support for the claim that the proportion of target behaviors occuring in windows after reinforcers is greater than tathe proportion of target behaviors when they can occur at any point in the observation series. Further scrutiny suggests that this test may be misleading due to the inflated recounted sample size. Given the same difference in the proportions (see the numerator of equation 5), the recounted sample size raises the effect of significance, compared to the test of the same proportions using same test with the original sample size. 
Table 7. Contingency table for average recounted frequencies

\begin{tabular}{|c|c|c|c|}
\hline & \multicolumn{2}{|c|}{ Observation } & \multirow[b]{2}{*}{ Totals } \\
\hline & $\begin{array}{l}\text { Target } \\
\text { (Green) }\end{array}$ & $\begin{array}{l}\text { Non-Target } \\
\text { (Not Green) }\end{array}$ & \\
\hline After & $\begin{array}{c}10 \\
(.7)^{\mathrm{a}}\end{array}$ & $\begin{array}{c}4.33 \\
(.3)\end{array}$ & $\begin{array}{c}14.3 \\
(1)[.59]\end{array}$ \\
\hline Before & $\begin{array}{c}4 \\
(.41)\end{array}$ & $\begin{array}{l}5.67 \\
(.58)\end{array}$ & $\begin{array}{c}9.67 \\
(1)\end{array}$ \\
\hline Totals & $\begin{array}{l}14.01 \\
(.58)\end{array}$ & $\begin{array}{c}10 \\
(.89)\end{array}$ & $\begin{array}{l}24 \\
(1)\end{array}$ \\
\hline
\end{tabular}

a. Values in parenthesis are cell values divided by the row totals (i.e. row-margin proportions)

Contingency tables for average recounted frequencies. The average frequency

table (see Table 7) serves the purpose of reducing the sample of the recounted series back to the original series, while retaining the proportions of events classified across all reinforcement sequences. Since this strategy retains approximately the same proportions as the recounted table, it will produce a more conservative $z$-score and p-value than the test on the recounted frequency table (see equation 5 for the example). This strategy is not without its caveats. Technically, this operation of dividing the cell values by the number of reinforcers should alter the distribtion since one observation is subtracted for each sub-series. The resulting distribution is no longer stricly binomial count response, and thus, is expected to lose power in comparison to a strict distribution of counts (see Reitan \& Nielsen, 2016 for a discussion of these implications). These limitations are noted in the discussion of this thesis (see Chapter 8) and is presented as a warning to user's of Reinforcinator web-app. 
Equation 4. Allison and Liker z-score for the average recounted frequencies

$$
\begin{gathered}
z_{1}=\frac{\operatorname{Pr}(Y=\text { Target } \mid x=\text { After })}{\sqrt{\frac{\operatorname{Pr}(Y=\text { Target }) \ldots(1-P r(Y=\text { Target }) \ldots i}{(N-1) * P(Y \mid x=\text { After })(1-P(Y \mid x=\text { After })}}} \\
=\frac{.7-.58}{\sqrt{\frac{.58 *(.42)(.41)}{24 *(.59)}}}=\frac{.12}{\sqrt{\frac{.144}{14.2}}}= \\
=1.19
\end{gathered}
$$

\begin{tabular}{|c|c|c|c|}
\hline & \multicolumn{2}{|c|}{ Observation } & \multirow[b]{2}{*}{ Totals } \\
\hline & $\begin{array}{l}\text { Target } \\
\text { (Green) }\end{array}$ & $\begin{array}{c}\text { Other } \\
\text { (Not Green) }\end{array}$ & \\
\hline After & $\begin{array}{l}10.49 \\
(.73)^{\mathrm{a}}\end{array}$ & $\begin{array}{l}3.85 \\
(.27)\end{array}$ & $\begin{array}{c}14.3 \\
(1)[.6]\end{array}$ \\
\hline Before & $\begin{array}{l}3.53 \\
(.36)\end{array}$ & $\begin{array}{l}6.84 \\
(.63)\end{array}$ & $\begin{array}{c}9.7 \\
(.99)^{*}\end{array}$ \\
\hline Totals & $\begin{array}{c}14.02 \\
(.58)\end{array}$ & 9.98 & 24 \\
\hline
\end{tabular}

Table 8. Contingency table for recomputed frequencies

Contingency table for recomputed frequencies. The recounted and average

frequency tables are taken directly from the recounted frequency table and do not account for any variation in proportions across the separate sub-series. The recomputed frequency table combines the row-conditioned proportions for each sub-series contingency table, along with the counts of the contingency table for average frequencies (See table 8 for a reference). Specifically, the row-conditioned proportions for each sub-series table are multiplied by the corresponding values of the contingency table for average frequencies. The resulting values are then summed across all the sub-series tables, to create a new contingency table for 're-computed' event frequencies. 
By accounting for the proportions across each sub-series, this table is argued to provide increased reliability over the average recounted frequencies (Kindermann, 2019). In this way, the z-statistic uses the same sample as the z-score for the average table, but slightly different values in the numerator (see equation 5 for the example). As with the average frequencies, caution should be taken when using this in a binomial test since the transformation no longer uses strict count responses.

Equation 5. Allison and Liker z-score for the recomputed frequencies

$$
\begin{gathered}
z_{1}=\frac{\operatorname{Pr}(Y=\text { Target } \mid x=\text { After })_{\ldots}-\operatorname{Pr}(Y=\text { Target })}{\sqrt{\frac{\operatorname{Pr}(Y=\text { Target }) \ldots(1-P r(Y=\text { Target }) \ldots !}{(N-1) * P(Y \mid x=\text { After })(1-P(Y \mid x=\text { After })}}} \\
=\frac{.73-.58}{\sqrt{\frac{.58 *(.42)(.4)}{24 *(.6)}}}=\frac{.73-.58}{\sqrt{\frac{.58 *(.42)(.4)}{24 *(.6)}}}=\frac{.15}{\sqrt{.0067}} \\
=1.82
\end{gathered}
$$




\section{Chapter 6}

\section{Methods 3: An Online Application for Detecting Reinforcers}

The primary goal of this project is to provide researchers with a non-experimental strategy for measuring reinforcers on their naturalistic observational datasets. This part of the chapter proposes an online application called the Reinforcinator that can serve this purpose. I describe the features and functions the application and begin by a brief overview of the R package Shiny (Chang, Cheng, Allaire, Xie, \& McPherson, 2016) used to create the graphical user interface.

Shiny Web-Applications. The Reinforcinator utilizes the $\mathrm{R}$ core language $(\mathrm{R}$ Core Team, 2016) and the Shiny package for web-applications (Chang, Cheng, Allaire, Xie, \& McPherson, 2016). Powered by the R language, Shiny web-applications are one way to distribute $\mathrm{R}$ code and functions via the web- without requiring the download of $\mathrm{R}$ software, nor knowledge of the R language. Shiny applications can import and exporting datasets and can utilize any existing package of functions from R's statistical library. The Reinforcinator uses a few exiting $\mathrm{R}$ packages and relies mostly on functions that $\mathrm{I}$ previously created for re-counting and testing the series of observations. By using minimal packages, this program will require less computing power, avoid conflicts between similar functions from multiple sources, and finally, be fully customizable, for ongoing edits and additions.

Graphical user interface. The graphical user interface (GUI) of the Reinforcinator comprises a single sidebar and a split center panel / window (shown in Figure 19). The option sidebar provides the main interactive component for users to 
upload files, specify target variables, and download recounted a recounted data-file. The center panel is divided into a section that provides a text overview of the program and a section that contains 11 output tabs and 1 glossary tab. The remainder of this chapter describes the various options for the sidebar as well as the output of the various tabs.

Figure 19. Reinforcinator Graphical User Interface

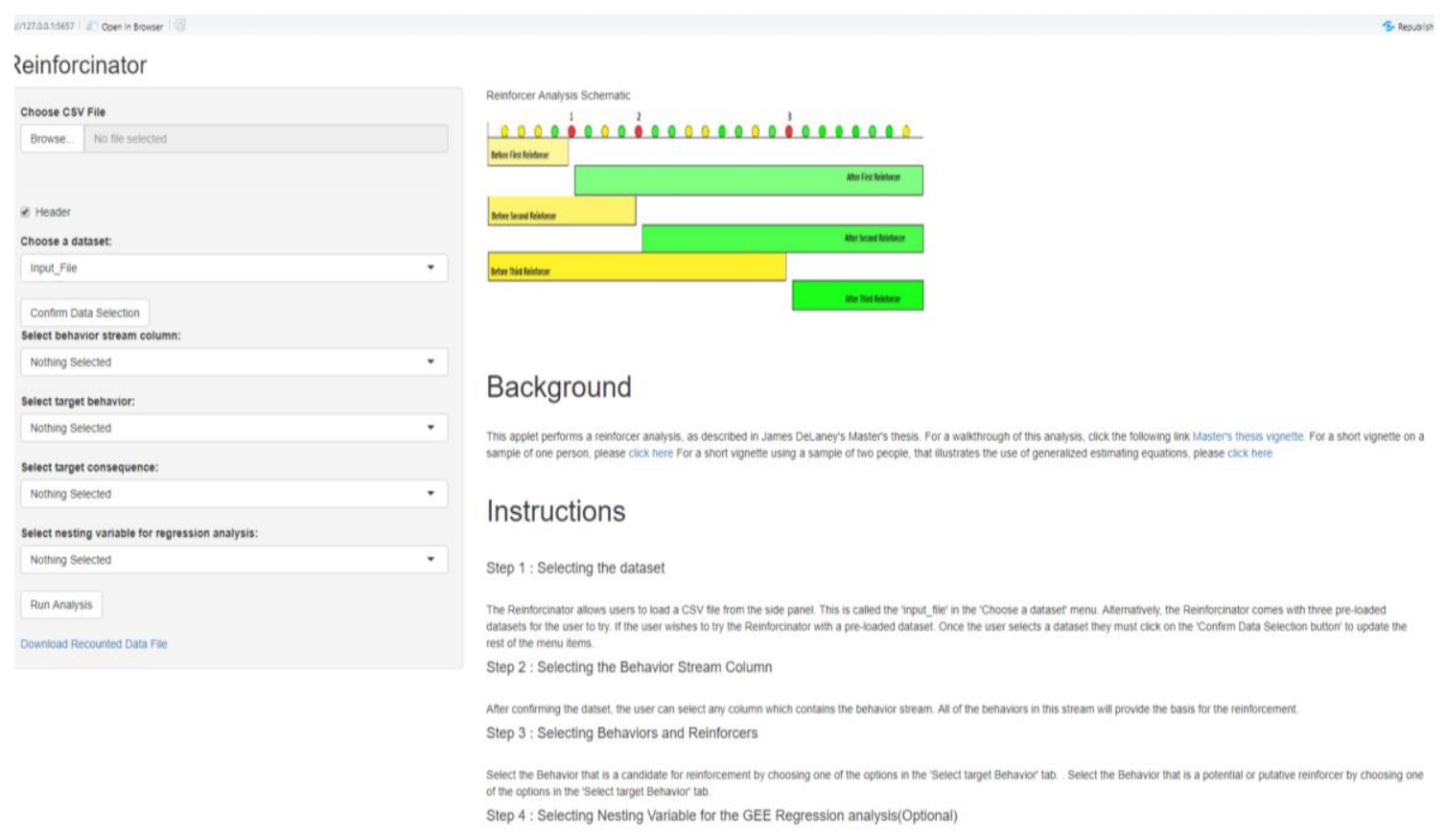

The Reinforcinator Sidebar Interface. The Reinforcinator is structured to begin with a raw csv file input which is used to populate the remaining options for selecting target behavior, reinforcer, and nesting variables for the contingency table and GEE analyses.

Input File. A data-file is necessary for any Reinforcer analysis. Users can upload a data-file by clicking on 'Choose CSV File' and selecting a file from their computer's directory. After loading the file, the user must select the 'Input_File' from the 'Choose a dataset' drop-down menu and then click on 'Confirm dataset' for the rest of the drop- 
down menus to populate. Alternatively, the user can select one of three example datasets from the 'Choose a dataset' drop-down menu. In any case, each datafile must be formatted in such a way that columns represent variables and rows represent observations.

Behavior stream and target variables. The columns of the data-file populate the options for 'Select the Behavior Stream Column' pull-down menu. The variable that is selected is assumed to contain all behavioral observations used to detect putative reinforcers. The unique values of the selected behavior stream populate the options for the 'Select Target Behavior' and 'Select Target Consequence' drop-down menus. The 'Target Behavior' is the behavior code that corresponds to the behavior being reinforced (i.e. the dependent variable) and the 'Target Consequence' is a putative reinforcer (i.e. independent variable), for which a before and after comparison is made. 
Figure 20. Reinforcinator text panel

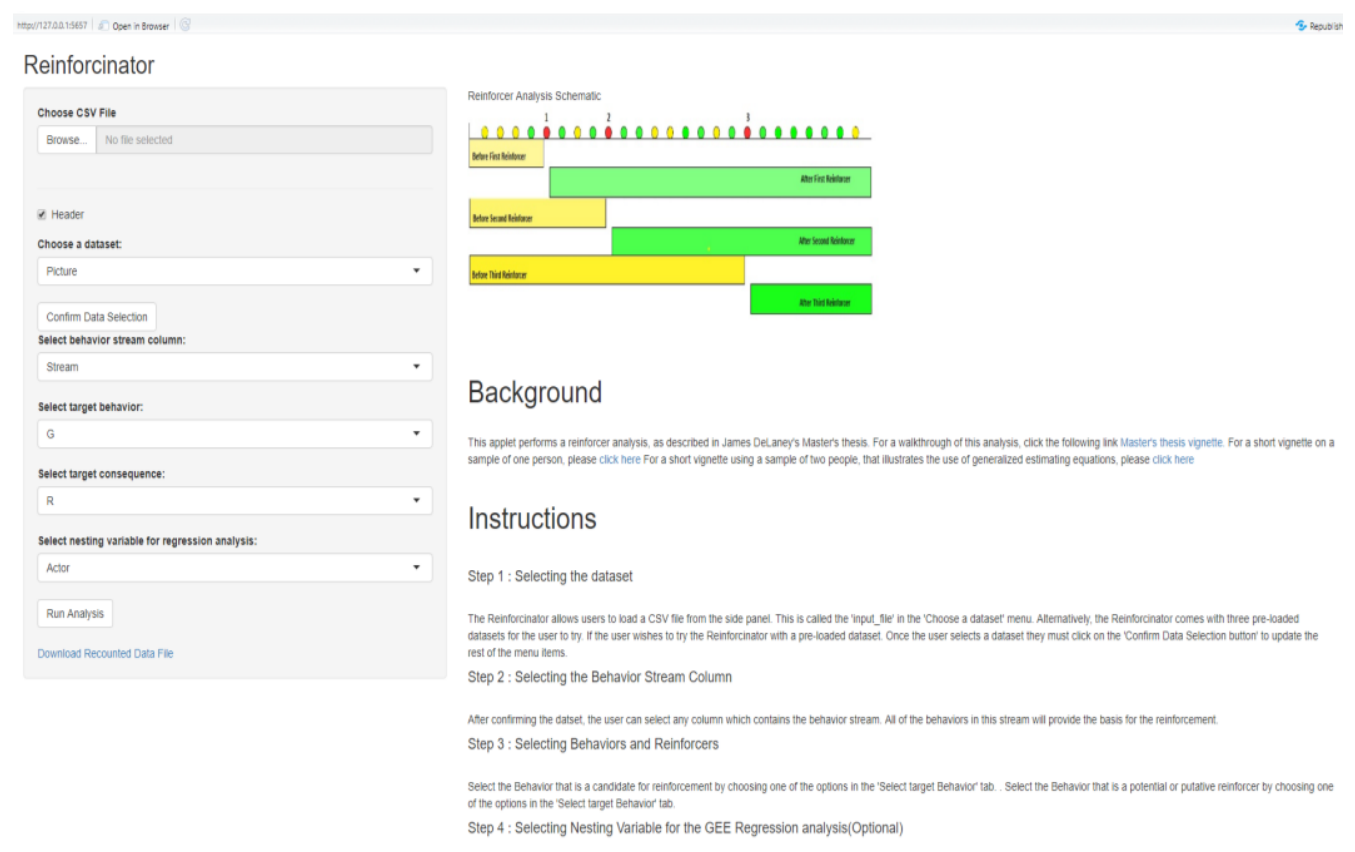

After selecting the target variables, the user is prompted to select a 'unit' or nesting variable for specifying the larger unit of repeated measures of the dependent variable. This nesting variable is necessary for specifying the 'ID' argument in geepack package in R (Halekoh, Højsgaard \& Yan, 2006); where 'ID' is synonymous with 'cluster' or 'nesting' variable in the parlance of repeated longitudinal data analysis. All primary analysis models in this thesis use 'focal child ID' as the 'nesting' or clustering variable (see Chapter 5 for details).

In addition to clusters, the geepack uses a 'waves' argument to specify the order of repeated measurements of the same unit and is recommended when each cluster is of a different size. The 'Sub-Series' variable is supplied to the 'waves' argument for the gee's used in the Reinforcinator in the backend of the program - regardless of whichever 
variable the user selects for the clustering variable. The waves argument is used to identify the order in which observations are clustered within the clustering variable (e.g. 'ID') that is used for repeat measures. Once the user selects the nesting variable, then they must click on 'Run Analysis' in order to populate the output tabs for all analyses.

Figure 21. Example of an appropriately reformatted dataset

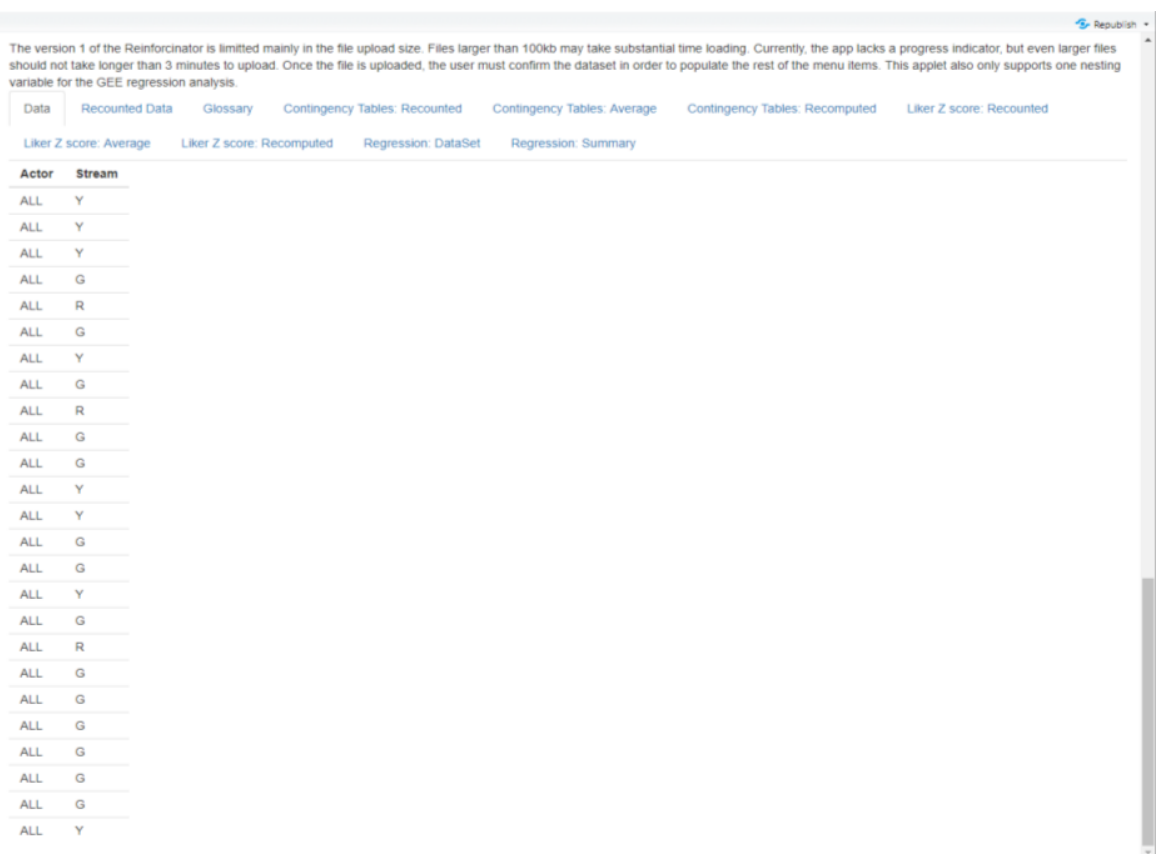


Figure 22. GEE analysis summary output

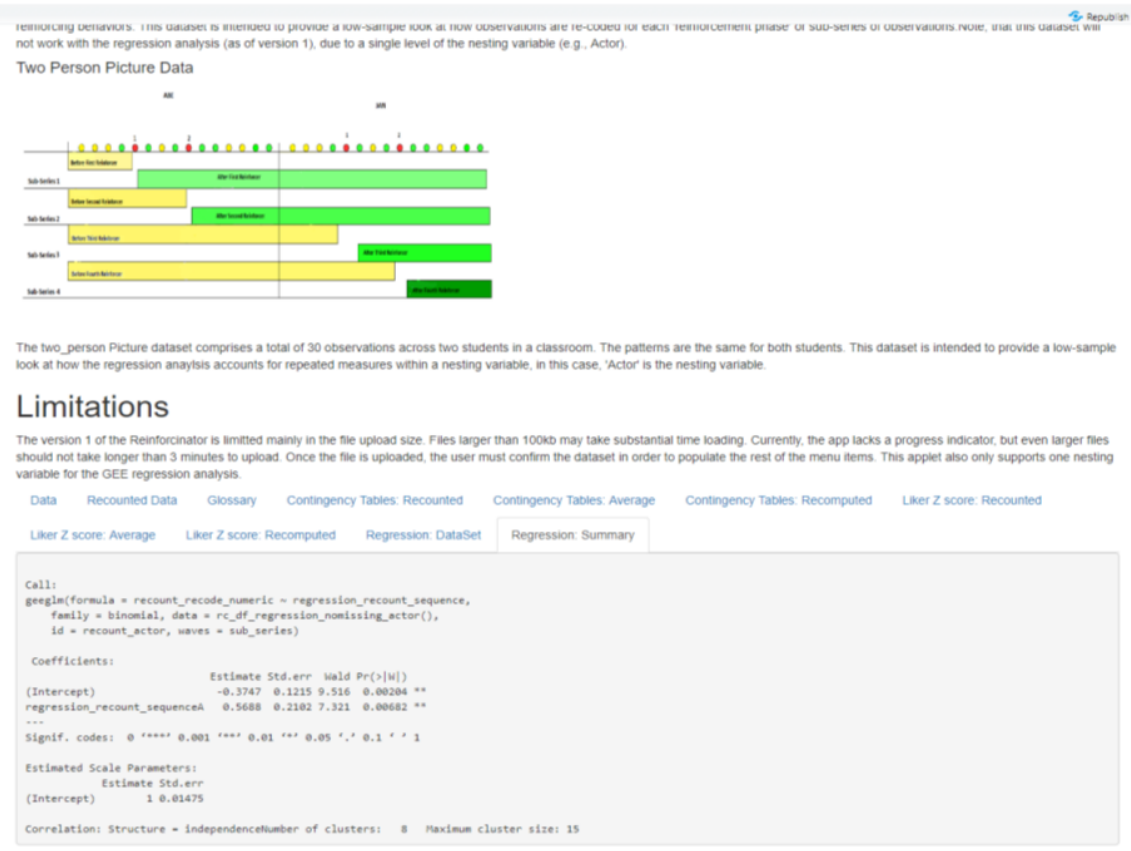

Output Summary. The bottom portion of the main window provides a total of 11 tabs, each with a unique output display. I organize this section by discussing the tabs into qualitatively similar sections.

Data Tabs. There are three 'Data tabs' which provide a data-frame outputs. The 'Data'tab simply shows the chosen dataset as a data-frame without any manipulations. The 'Recounted Data' tab shows the recounted data-file (see Chapter 5). This differs from the data-frame shown in the 'Data' tab in that it only contains the behavior stream variable from the original data-file and introduces the following variables from the Recounting procedure : 1) recount_stream, 2) sub_series, 3) recount_stream_index, 4) recount_recode_stream, 5) recount_sequence, 6) recode_stream_numeric, 7) 
regression_recount_sequence (see Appendix C for the glossary of these terms). The 'Regression Dataset' tab contains the same recounted data frame with the addition of a 'recount actor' variable. This variable contains the values of the selected nesting variable corresponding to each recounted observation from the original data-file. This is used for all statistical tests that account for the repeated observations.

Contingency Table Tabs. There are three contingency table tabs, each corresponding to each of the three contingency tables describing the recounted series (see Chapter 5). Each of these tables is presented as a matrix with 6 rows. The first three rows contain frequency counts and the last three rows contain the row conditioned proportions.

Figure 23. Recounted frequency table output

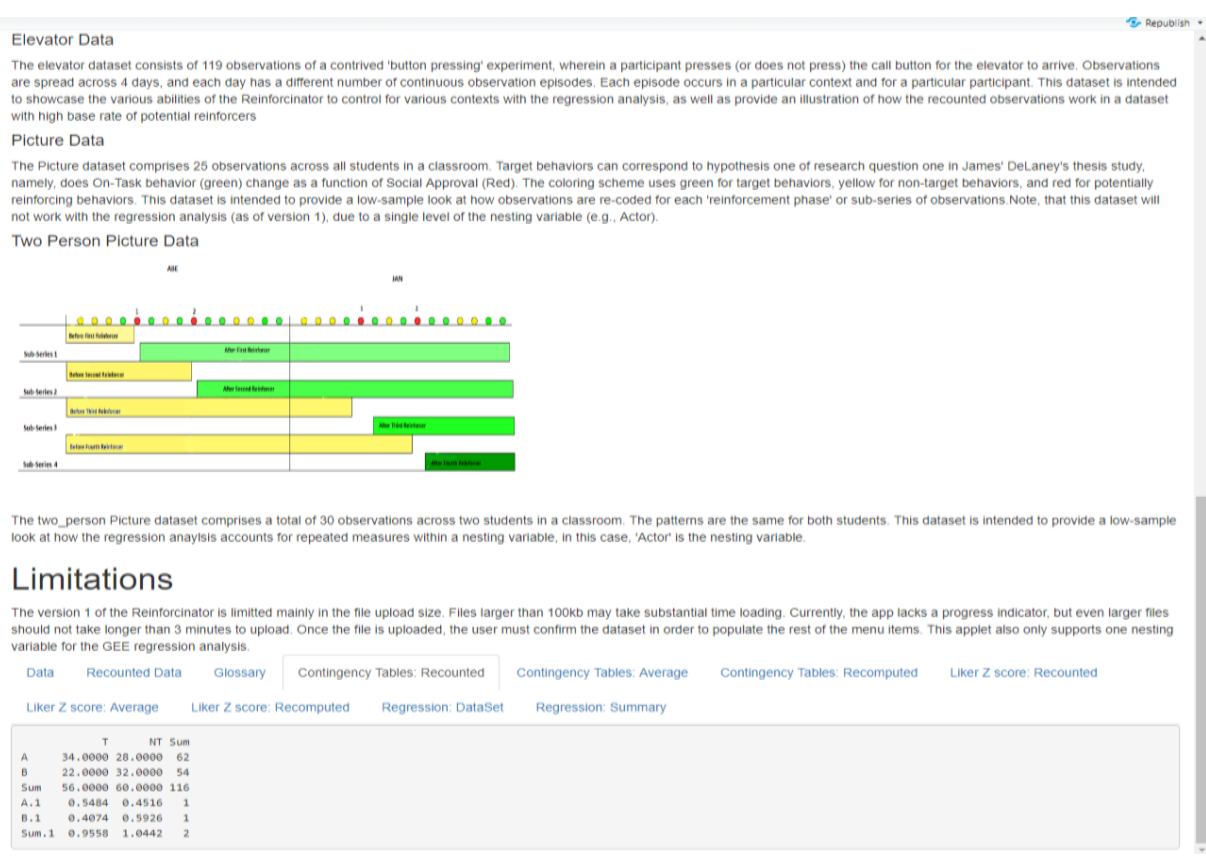

Contingency Table Test Tabs. The Reinforcinator provides the same binomial $z$ test for sequential dependence with the Allison and Liker (1982) correction that is used in 
the secondary analyses in this thesis (see Chapter 3 and 5 for details). This test compares the probability of target behavior in the after-reinforcement sequence to the overall baserate of target behaviors independent proportions for each of the three summary contingency tables. Using the notation from Gottman and Roy (1991), this test uses Equation 5, which shows the equivalence of the notational style used in Chapter 3 and that of Gottman and Roy, to compare the proportions of the target behavior in the afterreinforcement sequence (i.e., $\mathrm{P}_{\mathrm{T} \mid \mathrm{A}}$ or $\operatorname{Pr}(\mathrm{Y}=$ Target $\mid \mathrm{x}=$ After $)$ ) to the overall probability of target behaviors, that is, behaviors in both sequences (i.e., $\left(\mathrm{P}_{\mathrm{T}}\right.$ or $\operatorname{Pr}(\mathrm{Y}=$ Target)). This equation is adapted from Allison \& Liker (1982, pp. 395) for sequential dependency in a contingency analysis to the current analysis of reinforcers. In this case, $k$ $=1$ due to the removal of the reinforcing event in the contingency table.

Equation 6. Binomial z test for lagged dependence

$$
\begin{aligned}
& z_{1}=\frac{P_{T \mid A}-P_{T}}{\sqrt{\frac{P_{T}\left(1-P_{T}\right)\left(1-P_{A}\right)}{(N-k) * P_{A}}}} \\
& =\frac{\operatorname{Pr}(Y=\text { Target } \mid x=\text { After })_{1}-\operatorname{Pr}(Y=\text { Target })}{\sqrt{\frac{\operatorname{Pr}(Y=\text { Target })(1-P r(Y=\text { Target })}{(N-1) * P(Y \mid x=\text { After })(1-P(Y \mid x=\text { After })}}}
\end{aligned}
$$

Note, this test is an alternative to the GEE analysis and the standard error does not specifically account for repeated observations within a nesting variable (e.g. sub-series) or observations ordered within a nesting variable (e.g. child's ID). Additionally, this strategy does not account for the repeated observations due to recounting observations. 
Thus, the test will use the recounted $N$ when testing the contingency table of recounted series but will use the original $N$ for the contingency tables for the average and recomputed recounted frequencies.

Regression Summary Tab. This tab provides the output of the conditional regression summary that is fitted by general estimating equations (GEE; Liang \& Zeger, 1986) using the regression summary using the geepack package (Halekoh, Højsgaard \& Yan, 2006).As described in more detail in Chapter 5, general estimating equations account for dependency between events clustered under some secondary identification variable (e.g. ID) (Agresti, 2013, pp. 462). The geepack package contains a geeglm function which appropriates a general linear model formula to account for non-normal outcome variables that contain repeated measures. Because the recounting procedure creates a clustering by design from recounting the same events in the original series, the geeglm function uses the sub-series variable as the cluster variable.

As Halekoh, Højsgaard and Yan (2006) indicate, the standard gee model assumes that that observations are equally separated in time, and that observations are missing at random (i.e., MAR). The geeglm function in the geepack package can accommodate violations of this assumption through the waves argument - which allows users to indicate whether the observations are systematically ordered or missing by an additional variable. As explained earlier (see Chapters 2 and 3) the assumptions of the standard gee model are violated due to the nature of the naturalistic observations in this thesis; where observations are repeatedly measured within focal children and each focal child has a different number of event-coded observations within each three-minute observation episode. Because the sub-series preserves the order of events observed within focal 
children (see Chapter 5 for details), all gee models used in the primary analysis use focal child ID as the wave argument in the gee models.

The Reinforcinator allow the user to specify any variable for which observations are ordered by the clustering variable. Additionally, the program and primary analyses uses a binomial distribution for modelling the outcome variable. All models in the Reinforcinator are specified using 'recounted_regression' dataset - which ensures all observations are recounted while preserving the variable corresponding to 'waves' variable (as indicated by the user). I direct the interested reader to Appendix E for a brief technical summary of the regression models and Appendix D for their corresponding equations.

Distributing the Application Online. Currently, the Reinforcinator lives on a Shiny IO apps website (see https://delaneyj1786.shinyapps.io/reinforcinator_shiny/). The plan is to redistribute this application on a on a google-hosted website (see https://sites.google.com/site/sonetpsu/ for an example). Users can access this website in one of two ways: by directly entering the URL into a web-browser or by selecting a link on Dr. Thomas Kindermann's Portland State University's faculty web page (see https://www.pdx.edu/psy/thomas-kindermann-phd). Updates to the application based on planned revisions (see the Discussion chapter 8) will be posted on both websites. 


\section{Chapter 7}

\section{Results}

This section begins with a short summary of the analysis strategies described in Chapters 3 and 5 and then present the results of the analyses. Because the review section reiterates the material from Chapters 3 (see Contingency vs. Reinforcement in Classroom Observations) and Chapter 5 in its entirety, the reader is encouraged to skip this review if necessary. All results were obtained using the functions described in Chapter 6, outside the web-app and in R studio directly. This approach was taken as an alternative to using the web app directly, mainly because of the sample size of the recounted sample, and the computational requirements for the GEE models with such a large sample and cluster size (see Chapter 8 for a discussion on this issue). All primary hypotheses were tested using the general estimating equations via the geepack package (Halekoh, Højsgaard \& Yan, 2006). All secondary hypotheses were tested using binomial $z$-tests of independence for contingency tables, using the Allison and Liker (1982) correction for lagged dependence.

\section{Review of Analysis Strategies}

The analyses of this study assess reinforcers along a naturalistic stream of observations; specifically, the set of classroom observations used by Sage and Kindermann (1999) for their analysis of overall contingency patterns on children's naturally occuring classroom behaviors. Each analysis corresponds to a primary and secondary hypothesis. The primary hypotheses are based on Skinner's (1938) definitional criteria of reinforcers, and compare the target behavior probability (as determined by the 
hypothesis) across reinforcement sequences; that is, 'before' and 'after' reinforcers.

The secondary hypotheses draw inspiration from traditional tests of reinforcer contingencies via lag-sequential analyses (e.g., Bakeman and Gottman, 1997), and view the recounting procedure as an extension of lag-sequential analyses. Accordingly, these secondary tests compare the probability of target behaviors after reinforcers occur to the overall probability of the target behaviors, using the Allison and Liker (1982) z-test for sequentially dependent observations). The question for these analyses is then whether a putative reinforcer does have alter future behaviors, in an entire set of observations and across all individuals, in a way that distinguishes these observations from the others, in a way that is consistent with Skinner's operational definition of reinforcers (e.g. is the proportion of behavior greater following reinforcers, compared to the overall proportion).

All analyses test the 'recounted observation series', which is used to classify observations as 'targets' or 'non-targets', 'before' or 'after' reinforcers. The intent of this classification procedure is to re-structure a 'messy' set of observations, that contain behaviors intermixed with potential reinforcers, into a dataset where each observation can be categorized as a target or non-target behavior and before or after reinforcers. Stated differently, the purpose is to restructure a naturalistic set of observations so that a clear 'before' and 'after' probability comparison is possible without any experimental manipulations or major assumptions.

It is important to note again that the recounting procedure is applied using an 'overall strategy', such that observations are classified by their 'reinforcement sequence' (e.g., as occuring 'before' or 'after' reinforcers), without making any distinction between behaviors that came from separate focal children (i.e. separate individuals, in the general 
case). This increases the appeal of the strategy, so that researchers need less worry about individuals with especially small samples (e.g., participants who were not available for all days of an observation).

Figure 24. Reinforcement detection measure (a.k.a. recounting procedure) (Figure 1 reprint)

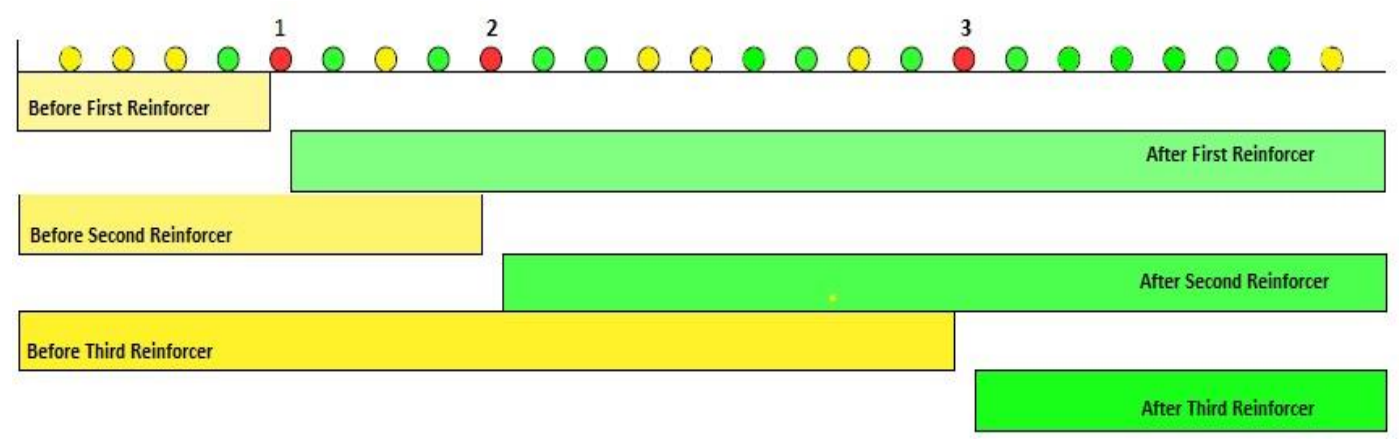

The recounting procedure (see Figure 24 for reference) classifies observations according to whether they occurred before or after a putative reinforcer. Then, it replicates the observation series repeatedly again for each further putative reinforcer in the data series, analogous to a moving average window. This is done for all occurring putative reinforcers, so that in each wave of replication, all observations can be cross classified as occurring before or after reinforcers. This strategy can be viewed as an extension of a lag-sequential analysis, since it classifies observations according to a sequential structure, and allows for a comparison across all observations and in both directions. However, by re-sampling the observations, this strategy introduces new dependencies on-top of the pre-existing dependency in the original observations (e.g. repeated observations within individuals). The primary and secondary analyses use different strategies to account for dependency structures and are reviewed in the 
following paragraphs.

Overview of reinforcement detection strategy. The goal of the thesis is to introduce the 'recounting procedure' as a tool to transform any set of behavioral observations so that they can be analyzed for indications of reinforcement patterns by classifying observations into reinforcement sequences (e.g., occurring 'before' or 'after' reinforcers), and as 'target behaviors' or 'non-target behaviors'. The procedure gives to usually imbalanced naturalistic observations an ordered time structure and simulates a situation as if just one prototypical reinforcer occurred and as if all observations came from a single focal person (see Figure 25 for reference of the step-wise process). This makes it possible to compare the classified behavior proportions in the originally 'messy' streams of observations (e.g., multiple reinforcers, unbalanced behaviors before and after reinforcers, unbalanced observations across participants, etc.). 
Figure 25. Stepwise depiction of the recounting procedure (Figure 7 reprint)

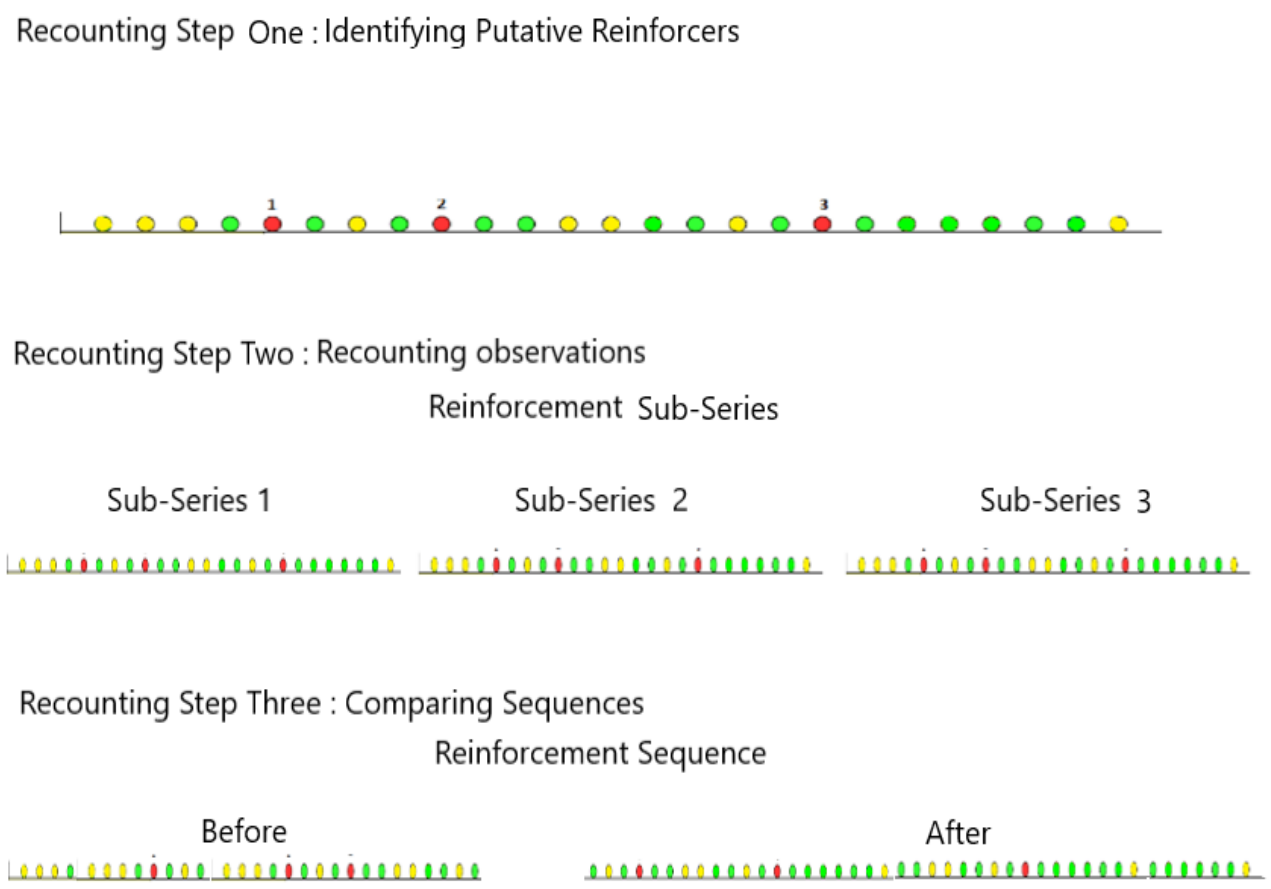

Readers may also see this as a strategy that makes it possible to create moving averages of proportions across the observation series. The proportions of the classified observations reflect the relative frequencies behaviors (coded as targets or non-targets as the dependent variable) with respect to the reinforcement sequence (coded as 'before' or 'after' reinforcers as the independent variable). To ensure each observation is classified correctly, that is, for each reinforcer in the series, observations are classified and indexed for each replication of the observation series (where I use the term 'sub-series' in reference to each replication).

Readers should note the terminology used to describe these processes. I use the term 'series' in reference to the original observations and the term 'recounted series', 
after applying the recounting procedure on the series. I use the term 'reinforcement sequence' as the variable which classifies recounted observations as 'before' or 'after' reinforcers. Readers should see the recounted series as an 'overall' observation series. In this way, all observations are classified with respect to reinforcers across all individuals (as is typical for observational datasets, interindividual differences will be rather large; and methods to account for these are discussed in the following sub-section).

Primary Analysis for Reinforcement Effects. The primary analyses test reinforcers directly, by comparing overall proportions of target behaviors, across reinforcement sequences (i.e., 'before' and 'after' reinforcement), in the recounted series. While the recounted series assumes that all observations come from a single 'individual', this assumption is ultimately false for the observations used in this thesis. To account for repeated observations within focal children in Sage and Kindermann's (1999) study, the primary analyses use general estimating equations implemented in the R software (Liang \& Zeger, 1986) package geepack (Halekoh, Højsgaard, \& Yan, 2006) to adjust the standard errors of the regression parameter estimates, in order to account for correlated repeated observations within focal children's observations, when making overall probability comparisons. This strategy fits the assumption of an overall analysis by measuring the outcome across all observations, while accounting for the fact that observations are repeated within individuals, and that recounted observations are thus repeated within individuals ordered by sub-series. As explained in Chapter 6, this is done by specifying individuals ID as a 'clustering' variable and sub-series as a 'wave', in the models implemented in R.

The method of general estimating equations adapts a generalized linear model to 
accommodate response variables with non-normal distributions (e.g., count data).

Particularly, the gee models in this thesis use a 'logit link' function to model the relation between reinforcement sequence and target behavior proportions. Consequently, the model output is expressed in terms of logits, but can be converted by readers using equations provided in Appendix D. Unlike typical logistic regression models, GEE's adjust parameter estimates and their standard errors using a 'working correlation structure', which accounts for the expected correlation between events under a 'clustering unit' or 'nesting variable'. The choice of a working correlation matrix determines a starting value (or 'best guess'), as to the observed intra-cluster correlations. Using an iterative procedure, this guess is updated based on the observed correlations. In this way, GEE's provide an efficient estimate for the standard errors, even if the working correlation is mis-specified (Wang, 2002).

All primary analysis models in this thesis use a general estimating equations approach with a logit link function to model the log odds of target behaviors given the reinforcement sequence, while adjusting for the average intra-individual correlations. Additionally, all models are specified with an 'independence' working correlation matrix to produce the smallest standard error and thus the most accurate p-value for the model coefficients. Unlike alternative structures (e.g., 'exchangeable'), the independence structure does not alter parameter estimates, and thus, the interpretation of the model parameters is the same as with a logistic regression analysis. All models are implemented in R with the geepack package (Halekoh, Højsgaard, \& Yan, 2006), and made available to the reader via the Reinforcinator web-app (see https://delaneyj1786.shinyapps.io/reinforcinator shiny/), made with the shiny package 
(Chang et. al., 2016).

\section{Secondary Analysis Strategies: Adaptation of Traditional Lag Sequential Analysis Methods and Delineation of Reinforcement Proportions}

The secondary analyses test reinforcers indirectly, by comparing the proportion of target behaviors after reinforcers to the overall base-rate of target behaviors. These analyses are inspired by lag-sequential analysis (e.g. Bakeman and Quera, 1996) and and follows from the original conceptualization of the recounting procedure extension of a lag-analysis to larger 'windows', in which the dependent variable is measured (aggregated) across all lags-ahead (after) and all lags before reinforcers. Following from the lag-sequential analysis, this secondary strategy compares proportions directly from contingency tables of the recounted series. Accordingly, this strategy adopts the Allison and Liker $z$-test for contingency tables, used to correct for sequential dependence across all recounted observations.

I apply the test to the $2 \times 2$ contingency table that classifies the dependent variable behavior (with categories target or non-target) by the independent variable reinforcement sequence (with categories before or after). While the table resembles a 2 x 2 contingency table for independent variables, the lag-sequential z-score is meant for tables on repeated measures, that is, where the rows represent the values of the dependent variable at the first measure and the columns represent the values of the dependent variable at the second measure. Accordingly, I begin this section by showing how the contingency table for the recounted series (called the recounted frequency table) is equivalent to a table where the cells represent the count of transitions across time for the dependent variable. 
The remainder of this section reviews the three contingency tables and the Allison and Liker $z$-score for lagged dependence.

Traditional contingency stable style tests for complete pairs. The Allison and Liker (1982) $z$-score has a long history in testing reinforcement hypotheses from naturalistic observations. In its original usage, this test compares the proportions of a potential reinforcer that immediately follow a target 'antecedent' behavior and compares this proportion to the overall 'base rate' proportion of times that the potential reinforcer occurs across any antecedents. Like the recounting procedure, observations are identified as 'antecedents' and 'consequences' across all individual identifiers. While the recounting procedure identifies antecedents and consequences across all time points before and after reinforcers, the typical lag-one analysis identifies observations as immediate consequences one step ahead of the antecedent - said to 'lag behind' by one unit. Figure 26 shows a general set of six observations where the index $t$ is used to label observations as antecedents (e.g., $X_{t}$ ), and then consequences (e.g., $\left.X_{t+1}\right)$.

Figure 26. $3 \times 3$ transition table for lag-one pairs (reprint)

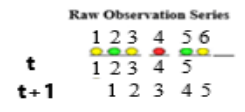

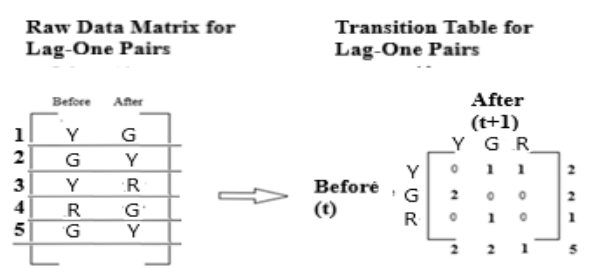

The traditional lag-one test uses the consequence at time $t+1$ as the dependent variable and counts all pairs of antecedents and consequences in the form $\left(Y_{t+1}, X_{t}\right)$. All pairs are then tabulated in a 'transition table', which shows the total number of times the 
a given category of the dependent variable was observed at time $t+1$ given that a category of the independent variable was observed at a lag of 1 unit of time earlier. The $3 \times 3$ transition table shown in Figure 26 can then be 'broken down' into a marginalized table to test hypotheses concerning any consequence given any antecedent behaviors.

Figure 27. 2 X2 marginalized table for directed lag-one contingency hypothesis (reprint)

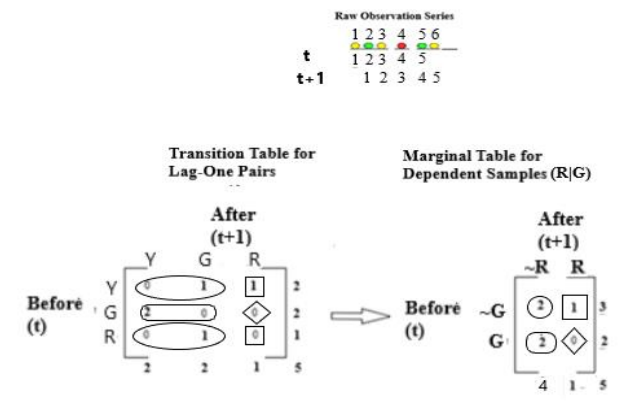

Figure 27 shows a 2 x 2 table used to test Sage and Kindermann's (1999) hypothesis that Social Approval (e.g. red circles) are more likely to follow On-Task behaviors (e.g. green circles) than all other antecedents. In this case, the $3 \times 3$ table is broken down by consolidating all non-target consequences (e.g., green and yellow circles) into one category (corresponding to non-target consequences), and all non-target antecedents (e.g., red and yellow circles) into another category (corresponding to nontarget antecedents). The cells of the 'Marginal Table' are computed by the sums of the combined behaviors, as the Figure depicts.

Equation 7. Allison and Liker Z-score (notation adjusted for contingency analysis)

$$
z_{1}=\frac{\operatorname{Pr}\left(Y_{t+1}=\operatorname{Target}\left(R^{8}\right) \mid X_{t}=\operatorname{Target}(G)\right)-\operatorname{Pr}(Y=\operatorname{Target}(R))}{\sqrt{\frac{\operatorname{Pr}(Y=\text { Target }(R))(1-P r(Y=\operatorname{Target}(R)))(1-\operatorname{Pr}(X=(\operatorname{Target}(G))}{(N-1) * \operatorname{Pr}(X=(\operatorname{Target}(G))}}}
$$

\footnotetext{
${ }^{8}$ Where R is red circles and G represents the green circles, as depicted in Figure 26.
} 
Finally, to test the hypothesis that red circles are more likely to follow green circles (versus any other antecedents), we compute the relevant proportions from the contingency table and enter them into the Allison and Liker $z$-score (see Equation 7). This equation compares the conditional probability of target consequences given target antecedents (e.g., red given green) to the unconditional probability of target consequences. The denominator accounts for the base rate proportions of red and green circles.

Limitations and Extensions of the Lag analysis for reinforcers. In its traditional usage, the lag analysis is limited on two fronts for addressing reinforcers. Firstly, it only looks at relations across one lag. That is, it only considers the dependent variable when it comes immediately after the target antecedents (e.g., lag 1, 2, 3, etc., assume random intervening observations). Translating to reinforcers, this means we would only test probability of On-Task behaviors immediately following Social Approval and not all future On-Task behaviors. This can be extended by averaging proportions across computations for each lag, but at the expense of extreme computational intensity.

A second limitation in lag analysis for reinforcer detection is that it computes the dependent variable lagging 'behind' or 'ahead' of the independent variable, at a time. This contrasts with the classification strategy in this thesis; which labels observations as occuring before and after reinforcers, across all lags. This latter strategy is analogous to classifying the dependent variable as lagging behind the independent variable (e.g. before reinforcement) and lagging ahead of the independent variable (e.g. after reinforcement). While the secondary analyses of this thesis are analogous to the tests in lag-sequential 
analyses (e.g., comparing probability of behaviors in after reinforcers to base-rates), the classification strategy is generally more versatile than a lag-sequential analysis, since it simultaneously classifies observations bi-directionally. The interested reader in directed to Appendix $\mathrm{G}$ for an example strategy of averaging across all lags in one direction and computing lags in both directions (using the example of lag-one).

Despite its limitations, ideas from the lag analysis can be used to guide a reinforcer analysis. For example, the lag-sequential analysis pairs observations sequentially, and not according to some higher order unit of analysis (e.g., observations within individuals). In this way, the dependency is due to re-sampling the same observations, and thus, the $z$-score accounts for this type of dependency. A second example is that this strategy can be used in messy datasets; where there are uneven number of observations before or after reinforcers, multiple reinforcers, or mixed number of reinforcers across participants. This contrasts heavily with similar tests (e.g., Mcnemar test for before and after comparisons for matched pairs), which pair observations according to individuals and assume complete observations at both time points. While this latter test can be used with some modifications (see Chapter 5 for a discussion), it falls outside the scope of the current thesis and is planned for future versions of the program as discussed in Chapter 8 .

Adapting the test to reinforcer analysis. The original conceptualization of the recounting procedure saw this strategy as a theoretical extension of a lag-sequential analysis. Statistically, the two techniques were envisioned differently. Instead of pairing observations by a lag, the recounting procedure simply classified observations via multiple 2 x 2 contingency tables for independent variables; where a separate table was 
made for each reinforcer sub-series, and events were classified into independent and mutually exclusive 'before' and 'after' categories, and as 'target' or 'non-target' behaviors. By combining multiple tables, tests on the resulting recounted frequency table could be made using the Allison and Liker $z$-test for dependency introduced by resampling the same observations. Furthermore, the recounted frequency table resembles the marginalized contingency table for lag analysis, but without requiring the computation of transitions or pairing observations 'before' and 'after' reinforcers.

Figure 28. Equivalency of marginal table of incomplete pairs and recounting procedure
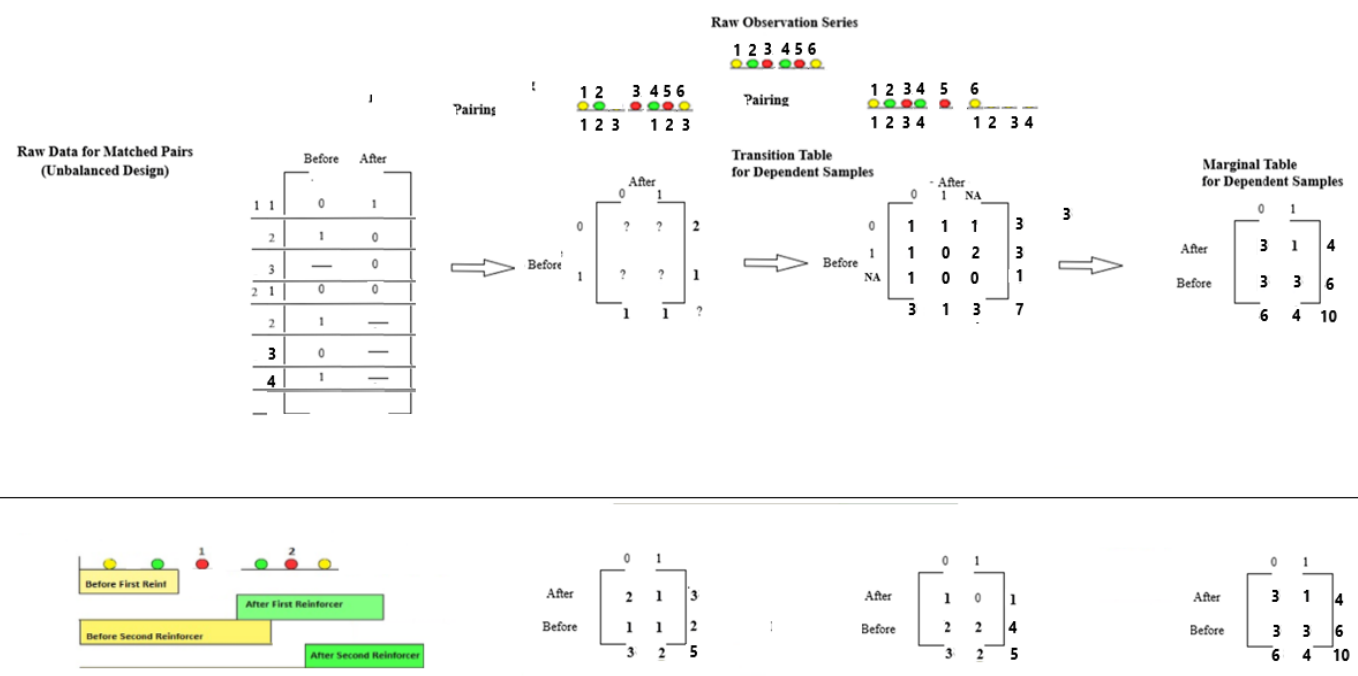

Figure 28 shows the equivalency of the tables produced by the recounting procedure and a strategy for creating 'before' and 'after' pairs on unmatched behavioral observations. The purpose of this figure is to further justify the secondary analyses, by showing that it is possible to conceive a reinforcer analysis in terms of transitions in paired observations. Unlike the lag-sequential analyses, pairs are created in reference to the location of each reinforcer in the original series. 
Figure 28 shows two reinforcers, corresponding to two sub-series, and thus, corresponding to two sets of sequential pairings. In each pairing, the index 'resets' when reinforcement occurs; such that the same index is used for observations before reinforcers and after reinforcers. In this way, the first observation before reinforcers pairs with the first observation after reinforcers, and so on and so forth. In this way, the pairing become analogous to matched pairs for scenarios such as voting outcomes; where the first observation before and after reinforcement would correspond to the votes of the first individual at two time points, and so on and so forth.

Since naturalistic observations cannot be expected to 'balance' across reinforcers, it is expected to have 'missing' observations across the pairs. Unlike a typical voting scenario, where voters who only submit a vote before some 'event' are not of interest and can easily be discarded from the analysis, each observation is of substantive interest for the reinforcer analysis. Accordingly, we can expand the $2 \times 2$ table in Figure 28 (where question marks are used to indicate incomplete pairs) to a $3 \times 3$ table that includes missing values. By including missing values, we can compute counts for 'unbalanced pairs', that is, where the sample size differs before and after reinforcers. We can then proceed to marginalize across the non-missing rows and columns, to obtain a $2 \times 2$ table the marginal distributions. The bottom portion of the figure shows how the same table is constructed from the sum of contingency tables for each sub-series, obtained by the recounting procedure. 
Figure 29. Reinforcement detection measure (Figure 1 reprint)

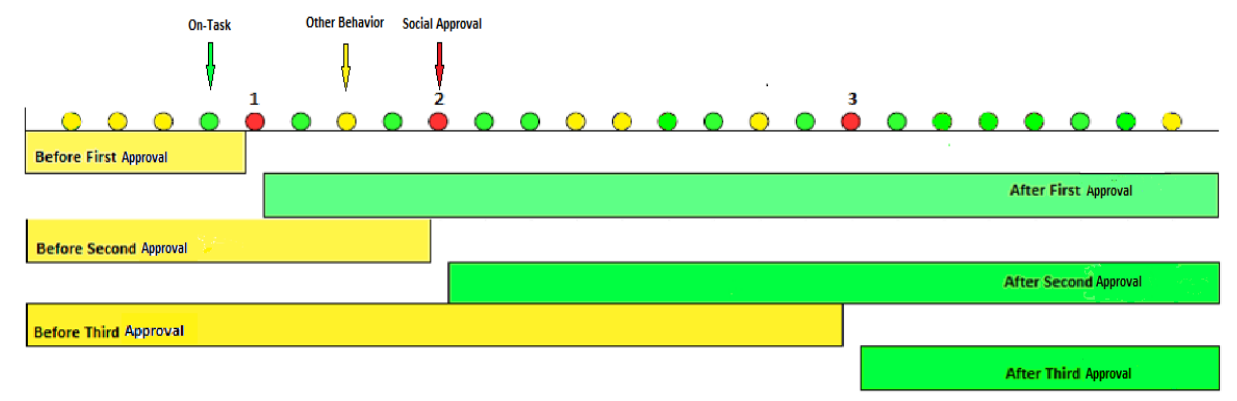

Brief review of the contingency tables of the overall recounting procedure. The bottom portion of Figure 28 showed the construction of the 'recounted frequency table', as the sum of $M$ contingency tables used to cross-classify the dependent variable (i.e., target behaviors) by the categories of the independent variable (e.g. reinforcement sequence). Table 9 shows this table again using a reprint of the original example (see Figure 29) used to introduce the overall recounting procedure earlier in this thesis. As an overall strategy, this table is used to address the lag-sequential hypothesis in terms of reinforcers; that is, whether the proportion of target behaviors 'after reinforcement' is greater than the proportion over the whole series.

Table 9. Contingency table for recounted frequencies (table 6 reprint)

\begin{tabular}{|c|c|c|c|}
\hline \multirow[b]{3}{*}{ After Red } & \multicolumn{2}{|c|}{ After Reinforcer } & \multirow[b]{2}{*}{ Totals } \\
\hline & Green & Not Green & \\
\hline & $\begin{array}{c}30 \\
(.7)^{\mathrm{a}}\end{array}$ & $\begin{array}{c}13 \\
(.3)\end{array}$ & $\begin{array}{l}43 \\
\text { (1) [.6] }\end{array}$ \\
\hline Before Red & $\begin{array}{c}12 \\
(.41)\end{array}$ & $\begin{array}{c}17 \\
(.59)\end{array}$ & $\begin{array}{l}29 \\
(1)\end{array}$ \\
\hline Frequency & $\begin{array}{c}42 \\
(.58)\end{array}$ & $\begin{array}{c}30 \\
(.42)\end{array}$ & $\begin{array}{l}72 \\
(1)\end{array}$ \\
\hline
\end{tabular}

As the examples illustrate, the contingency table for recounted frequencies is useful 
for providing accurate proportions that account for messy naturalistic scenarios (e.g., uneven observations before or after reinforcers, multiple reinforcers, etc.). When using these values in a test statistic such as the Allison and Liker $z$-score, this table may less than ideal due to the inflated sample size of the recounted series (recall that the total recounted sample is $M \times N$, where $M$ is the total number of reinforcers across the entire series). This has the effect of increasing the $z$-score based on a recounted sample size and providing a greater risk of type 1 error by not controlling for the re-sampling.

Table 10. Contingency table for average frequencies (table 7 reprint)

\begin{tabular}{|c|c|c|c|}
\hline \multirow{3}{*}{ After Red } & \multicolumn{2}{|c|}{ After Reinforcer } & \multirow[b]{2}{*}{ Totals } \\
\hline & Green & Not Green & \\
\hline & $\begin{array}{c}10 \\
(.7)^{\mathrm{a}}\end{array}$ & $\begin{array}{c}4.33 \\
(.3)\end{array}$ & $\begin{array}{c}14.3 \\
(1)[.59]\end{array}$ \\
\hline Before Red & $\begin{array}{c}4 \\
(.41)\end{array}$ & $\begin{array}{l}5.67 \\
(.58)\end{array}$ & $\begin{array}{l}9.67 \\
(1)\end{array}$ \\
\hline Frequency & $\begin{array}{c}14.01 \\
(.58)\end{array}$ & $\begin{array}{c}10 \\
(.89)\end{array}$ & $\begin{array}{l}24 \\
(1)\end{array}$ \\
\hline
\end{tabular}

The average frequency contingency table attempts to correct for the effects of resampling by dividing the values of the recounted frequency table by the number of reinforcers $M$. This serves the advantage of providing the same approximate proportions as the recounted table, while using the original sample of observations. In this way, tests using this table are expected to reflect a more accurate effect of reinforcement in messy situations (e.g., multiple reinforcers, etc.) across the original series. Finally, the recomputed frequency contingency table is postulated to be the most reliable of the three strategies. This approach accounts for the row-conditional proportions of each sub-series, 
as well as the overall cell average (taken from the average frequency table). Specifically, the cells of this table are the product of each sub-series conditional probability (for a given cell) and the value of the average table, summed across each sub-series (see Table 11 for the example).

Table 11. Contingency table for recomputed frequencies (table 8 reprint))

\begin{tabular}{|c|c|c|c|}
\hline & \multicolumn{2}{|c|}{ After Reinforcer } & \multirow[b]{2}{*}{ Totals } \\
\hline & Green & Not Green & \\
\hline After Red & $\begin{array}{l}10.49 \\
(.73)^{\mathrm{a}}\end{array}$ & $\begin{array}{l}3.85 \\
(.27)\end{array}$ & $\begin{array}{c}14.3 \\
(1)[.6]\end{array}$ \\
\hline Before Red & $\begin{array}{l}3.53 \\
(.36)\end{array}$ & $\begin{array}{l}6.84 \\
(.63)\end{array}$ & $\begin{array}{c}9.7 \\
(.99)\end{array}$ \\
\hline Frequency & $\begin{array}{c}14.02 \\
(.58)\end{array}$ & 9.98 & 24 \\
\hline
\end{tabular}

a. Proportions are taken from the row margins

Table 12 shows the values necessary for the z-score equation for each of the three contingency tables. The recounted table gives an inflated z-score, despite testing the same difference as the contingency table for average frequencies. The average z-score provides a more conservative $\mathrm{z}$ score due to using the correct sample size. The re-computed $z$ score sits in-the most conservative between the other $z$-scores and would be taken as support of the hypothesis that proportion of target behavior after reinforcement is greater than the chance base rate without prior knowledge of any reinforcement sequence 
Equation 8. Allison and Liker z-score adapted to reinforcer analysis (re-print)

$$
z_{1}=\frac{\operatorname{Pr}(Y=\text { Target } \mid x=\text { After })-\operatorname{Pr}(Y=\text { Target })}{\sqrt{\frac{\operatorname{Pr}(Y=\text { Target })(1-\operatorname{Pr}(Y=\text { Target }))(1-P(Y \mid x=\text { After })}{(N-1) * P(Y \mid x=\text { After })}}}
$$

Table 12. Proportions used in Allison and Liker (1982) z-scores

Recounted Table

$$
\frac{.7-.58}{\sqrt{\frac{.58 *(.42)(.4)}{71 *(.6)}}}=2.51
$$

$\operatorname{Pr}($ Green)

$\operatorname{Pr}($ After $)$

$\operatorname{Pr}($ Green $\mid$ Red $)$

Average Table

$\operatorname{Pr}$ (After)

$\operatorname{Pr}($ Green $\mid$ Red $)$

Recomputed Frequency Table

$\operatorname{Pr}($ Green $)$

$\operatorname{Pr}($ After $)$

$\operatorname{Pr}($ Green $\mid$ Red $)$ $\frac{42}{72}(.58)$

$\frac{43}{72}(.6)$

$\frac{30}{72}(.7)$

$$
\begin{gathered}
\frac{14.01}{24}(.58) \\
\frac{14.3}{24}(.59) \\
\frac{10}{24}(.7)
\end{gathered}
$$

$$
\frac{.7-.58}{\sqrt{\frac{.58 *(.42)(.41)}{24 *(.59)}}}=1.46
$$

$\frac{.73-.58}{\sqrt{\frac{.58 *(.42)(.4)}{24 *(.6)}}}=1.22$

$\frac{14.01}{24}(.6)$

$\frac{14.3}{24}(.6)$

$\frac{10}{24}(.7)$ 


\section{Results}

I present the results in three steps. I begin by overviewing the descriptive statistics for each distribution for each research question (starting with the original data file). I then detail the primary analysis results and follow this with a summary of the secondary analysis results. I repeat this reporting process for each hypothesis.

Descriptive Statistics. The Sage and Kindermann (1999) data-file contained a total of 12,110 observations. These observations were spread across 25 focal children, across 11 daily observation episodes. There was a total of 135 missing observations across all focal children. Table 13 shows the distribution of all behaviors across the full observation stream, without removing missing observations.

Table 13. Distribution of overall behaviors in original data-file

\begin{tabular}{lccc}
\hline \multicolumn{1}{c}{ Behavior (Code) } & $\mathbf{N}$ & Percentage & Valid Percentage $^{\mathrm{a}}$ \\
\hline Approval (A) & 249 & $2.1 \%$ & $2.1 \%$ \\
Cooperation (C) & 1720 & $14.2 \%$ & $14.4 \%$ \\
Disapproval (D) & 48 & $.4 \%$ & $.4 \%$ \\
Factual Disagreement (DIS) & 33 & $.3 \%$ & $.3 \%$ \\
Ignore (I) & 101 & $.8 \%$ & $.8 \%$ \\
Move (M) & 236 & $1.9 \%$ & $2.0 \%$ \\
On-Task Active (OA) & 1957 & $16.2 \%$ & $16.3 \%$ \\
On-Task Passive (OP) & 6171 & $51 \%$ & $51.5 \%$ \\
Prompt (P) & 323 & $2.7 \%$ & $2.7 \%$ \\
Off-Task Active (XA) & 375 & $3.1 \%$ & $3.1 \%$ \\
Off-Task Passive (XP) & 762 & $6.3 \%$ & $6.4 \%$ \\
Missing (NA) & 135 & $1.1 \%$ & $2.1 \%$ \\
\hline a. Valid percentages are calculated by removing missing values from the total
\end{tabular}

The 135 missing observations were removed prior to recounting the overall behavior streams for each research question. This brought the total number of eligible observations recounting to 11,975 . The formula $N$ x $M$ was used to obtain the total number of recounted observations, where $M$ is the number of cases of each reinforcer, as determined by each research question and $N$ is the total number of observations (see 
Chapter 5). Because the length of the recounted series varies depending on the independent variable (i.e., putative reinforcer), I report the recounted series sample size separately for each research question.

\section{Research Question 1}

Research Question 1 asks whether social approval is a reinforcer for classroom behaviors. To answer this question the original file was scanned using the recounting procedure which duplicated the original observation stream for each of the 249 observations of Social Approval. This resulted in a total of 2,981,775 observations in the recounted behavior stream used to address Research Question 1.

Table 14. Distribution of recounted behaviors for social approval as a reinforcer

\begin{tabular}{lcc}
\hline \multicolumn{1}{c}{ Behavior } & $\mathrm{N}$ & $\%$ \\
Approval (A) & 6,200 & $2.1 \%$ \\
Cooperation (C) & 428,280 & $14.4 \%$ \\
Disapproval (D) & 11,952 & $.4 \%$ \\
Factual Disagreement (DIS) & 8,217 & $.3 \%$ \\
Ignore (I) & 25,149 & $.8 \%$ \\
Move (M) & 58,764 & $2.0 \%$ \\
On-Task Active (OA) & 487,293 & $16.3 \%$ \\
On-Task Passive (OP) & $1,536,579$ & $51.5 \%$ \\
Prompt (P) & 80,427 & $2.7 \%$ \\
Off-Task Active (XA) & 93,375 & $3.1 \%$ \\
Off-Task Passive (XP) & 189,738 & $6.4 \%$ \\
Totals & $2,925,974$ & $100 \%$ \\
\hline
\end{tabular}

Hypothesis 1. Hypothesis 1 states that Social Approval is a reinforcer for children's On-Task classroom behaviors. The primary test of this hypothesis compares the proportion of On-Task behaviors before and after Social Approval across all observations, under the expectation that On-Task behaviors after reinforcers will be greater than the proportion before reinforcers. A GEE model was specified with a logit 
link for the mean and variance of a binomially distributed outcome, with an independence working correlation structure for repeated measures.

The observed proportion of On-Task behavior before Social Approval was .161 and the observed proportion of On-Task behavior after Social Approval was .166. The difference of .005 between these two proportions was significant using the GEE model $\left(\beta_{1}=.0317\right.$, Wald $\left.\chi^{2}(1)=28.4, S E=.0059, p<.001\right)$. These findings suggest that OnTask behavior is $.05 \%$ more likely to occur after Social Approval occurs, across all students. This primary analysis supports the hypothesis that Social Approval reinforces On-Task behaviors by a direct comparison of before and after behavior proportions in the entire series of observations, while adjusting for the dependent observations within individuals.

Table 15. Research question 1 hypothesis 1 primary analysis

\begin{tabular}{cccc}
\hline Beta & $\begin{array}{c}\text { Coefficient Estimate } \\
\text { (Probability) }\end{array}$ & SE & Wald $\chi^{2}(1)$ \\
\hline$\beta_{0}$ & -1.648 & .004 & $156107^{* * *}$ \\
$\beta_{1}$ & .0318 & .006 & $28.4^{* * *}$ \\
\hline$* * *$ indicates $p<.001$ & & &
\end{tabular}

A secondary analysis was conducted to test reinforcers using a traditional Allison and Liker (1982) z-test of contingencies, adapted for the analysis of reinforcers. This test compared the probability of On-Task behaviors following reinforcers to the overall base rate proportion of On-Task behaviors. The test was performed on the contingency table for recounted frequencies, the average frequencies, and the re-computed frequencies (see the review in this chapter, or chapters 5 for a description of these tables). 
Table 16. Research question 1 hypothesis 1 proportions

\begin{tabular}{lccccc}
\hline $\begin{array}{l}\text { Summary } \\
\text { Series }\end{array}$ & $\mathrm{p}(\mathrm{Y}=\mathrm{OA} \mid \mathrm{X}=$ After $)$ & $\mathrm{p}(\mathrm{Y}=\mathrm{OA})$ & $\mathrm{p}(\mathrm{X}=\mathrm{After})$ & $N$ & $z$ \\
\hline Recounted & .166 & .163 & .488 & $2,981,526$ & 13.5 \\
Average & .166 & .163 & .488 & 11,974 & .868 \\
Recomputed & .166 & .161 & .488 & 11,974 & 1.45 \\
& & & & &
\end{tabular}

The Allison and Liker $z$-test (1981) found that the proportion of On-Task behaviors following reinforcement was significantly greater than the overall proportion across the recounted observation series $(\operatorname{diff}=.03, z=13.5, \mathrm{p}<.001)$. When dividing this distribution by the total number of reinforcers, the same difference was not significant for these average frequencies ( $\operatorname{diff}=.03, z=.868, \mathrm{p}>.05)$. Similarly, the difference was not significant across the recomputed frequencies at the .05 significance level (diff $=.05, z=$ $1.45, p>.05)$.

These secondary analyses provide almost no support at all for the hypothesis that Social Approval demonstrates a reinforcing effect for children On-Task behaviors, under a traditional test used in lag sequential analysis, for the three summary contingency tables. Table 17 shows the proportions used in the $\mathrm{z}$ score computations for each of the three contingency tables

Table 17. Research question 1 hypothesis 1 summary secondary analysis

\begin{tabular}{|c|c|c|c|c|}
\hline \multirow[b]{2}{*}{$\begin{array}{l}\text { Summary } \\
\text { Series }\end{array}$} & \multirow[b]{2}{*}{$\begin{array}{l}\text { Reinforcement } \\
\text { Sequence }\end{array}$} & \multicolumn{2}{|c|}{ On- Task Behavior } & \multirow[b]{2}{*}{ Total } \\
\hline & & Yes & No & \\
\hline \multicolumn{5}{|l|}{ Recounted } \\
\hline & After & $241,254(.166)$ & $1,215,065(.834)$ & $1,456,319$ \\
\hline & Before & $246,039(.161)$ & $1,279,168(.839)$ & $1,525,207$ \\
\hline & Total & 487,293 & $2,494,233$ & $2,981,526$ \\
\hline
\end{tabular}


Average

$\begin{array}{lccc}\text { After } & 969(.166) & 4880(.834) & 5849 \\ \text { Before } & 988(.161) & 5,137(.839) & 6,125 \\ \text { Total } & 1,957 & 10,017 & 11,974\end{array}$

Recomputed

$\begin{array}{lccc}\text { After } & 970(.166) & 4,879(.834) & 5,849 \\ \text { Before } & 954(.156) & 5,172(.844) & 6,125 \\ \text { Total } & 1,924 & 10,051 & 11,974\end{array}$

a. Proportions are computed from the row margins

Hypothesis 2. Hypothesis 2 states that Social Approval is a reinforcer for children's Off-Task classroom behaviors. The primary test of this hypothesis compares the proportion of Off-Task behaviors before and after Social Approval across all observations, under the expectation that Off-Task behaviors after reinforcers will be greater than the proportion before reinforcers. A GEE model was specified with a logit link for the mean and variance of a binomially distributed outcome, with an independence working correlation structure for repeated measures.

The observed proportion of Off-Task behavior before Social Approval was .024 and the observed proportion of Off-Task behavior after Social Approval was .039. The difference of .015 between these two proportions was significant using the GEE model $\left(\beta_{1}=.0525\right.$, Wald $\left.\chi^{2}(1)=1,361, S E=.0142, p<.001\right)$. These findings suggest that OffTask behavior is $1.5 \%$ more likely to occur after Social Approval occurs, across all students. This primary analysis supports the hypothesis that Social Approval reinforces Off-Task behaviors by a direct comparison of before and after behavior proportions, while adjusting for the dependent observations within individuals. 
Table 18. Research question 1 hypothesis 2 primary analysis

\begin{tabular}{cccc}
\hline Beta & Estimate & $S E$ & Wald $\chi^{2}(1)$ \\
\hline$\beta_{0}$ & -3.72 & .011 & $113,056^{* * *}$ \\
$\beta_{1}$ & .525 & .014 & $1,361^{* * *}$ \\
\hline$a . \quad * *$ indicates & $<.001$ & &
\end{tabular}

A secondary analysis was conducted to test reinforcers using a traditional Allison and Liker (1982) z-test of contingencies, adapted for the analysis of reinforcers. This test compared the probability of Off-Task behaviors following reinforcers to the overall base rate probabilities of Off-Task behaviors. The test was performed on the contingency table for recounted frequencies, the average frequencies, and the re-computed frequencies.

Table 19. Research question 1 hypothesis 2 proportions

\begin{tabular}{lccccc}
\hline $\begin{array}{l}\text { Summary } \\
\text { Series }\end{array}$ & $\mathrm{p}(\mathrm{Y}=\mathrm{XA} \mid \mathrm{X}=\mathrm{After})$ & $\mathrm{p}(\mathrm{Y}=\mathrm{XA})$ & $\mathrm{p}(\mathrm{X}=\mathrm{After})$ & $\mathrm{N}$ & $z$ \\
\hline Recounted & .039 & .0313 & .488 & $2,981,526$ & 77.8 \\
Average & .039 & .0313 & .488 & 11,974 & 4.93 \\
& .041 & .0311 & .488 & 11,974 & 6.16 \\
Recomputed & & & & & \\
\hline
\end{tabular}

The Allison and Liker $z$-test (1981) found that the proportion of Off-Task behaviors following reinforcement was significantly greater than the overall proportion across the recounted observation series $($ diff $=.007, z=27, \mathrm{p}<.001)$, the average frequencies $($ diff $=.007, z=4.72, \mathrm{p}<.001)$, and the recounted frequencies (diff $=.009, z$ $=6.01, p<.001)$. These secondary analyses provide additional and unanimous support for the hypothesis that Social Approval demonstrates a reinforcing effect for children's Off- 
Task behaviors, under a traditional test used in lag sequential analysis, for the three summary contingency tables. Table 20 shows the proportions used in the $\mathrm{z}$ score computations for each of the three contingency tables.

Table 20. Research question 1 hypothesis 2 summary secondary analysis

\begin{tabular}{llccc}
\hline \multirow{2}{*}{$\begin{array}{l}\text { Summary } \\
\text { Series }\end{array}$} & $\begin{array}{l}\text { Reinforcement } \\
\text { Sequence }\end{array}$ & \multicolumn{2}{c}{ Off Task Behavior } & Total \\
\cline { 3 - 4 } & After & $57,297(.039)$ & $1,399,022(.961)$ & $1,456,319$ \\
& Before & $36,078(.024)$ & $1,489,129(.976)$ & $1,525,207$ \\
& Total & 93,375 & $2,888,151$ & $2,981,526$ \\
Average & After & $230(.039)$ & $5,619(.961)$ & 5,849 \\
& Before & $145(.024)$ & $5,980(.976)$ & 6,125 \\
& Total & 375 & 11,599 & 11,974 \\
Recomputed & After & $239(.041)$ & $5,610(.959)$ & 5,849 \\
& Before & $133(.022)$ & $5,992(.978)$ & 6,125 \\
& Total & 372 & 11,602 & 11,974 \\
\hline
\end{tabular}

a. Proportions are computed from the row margins

\section{Research Question 2}

Research Question 2 asks whether direct Disapproval is a punisher $\mathrm{r}$ for classroom 
behaviors. Thus, the observations were recounted according to the 48 observations of Social Disapproval in the original data-file. There was a total of 307,800 recounted observations for addressing Research Question 1 (see Table 10).

Table 21. Distribution of recounted behaviors for direct disapproval as a punisher

\begin{tabular}{lcc}
\hline \multicolumn{1}{c}{ Behavior } & $N$ & $\%$ \\
\hline Approval (A) & 11,952 & $2.1 \%$ \\
Cooperation (C) & 82,560 & $14.4 \%$ \\
Disapproval (D) & 2,304 & $.4 \%$ \\
Factual Disagreement (DIS) & 1,584 & $.3 \%$ \\
Ignore (I) & 4,848 & $.8 \%$ \\
Move (M) & 11,328 & $2.0 \%$ \\
On-Task Active (OA) & 93,936 & $16.3 \%$ \\
On-Task Passive (OP) & 296,208 & $5.1 \%$ \\
Prompt (P) & 15,504 & $2.7 \%$ \\
Off-Task Active (XA) & 18,000 & $3.1 \%$ \\
Off-Task Passive (XP) & 36,576 & $6.4 \%$ \\
Totals & 306,216 & $100 \%$ \\
\hline
\end{tabular}

Hypothesis 3. Hypothesis 3 states that Social Disapproval is a punisher for children's Off-Task classroom behaviors. The primary test of this hypothesis compares the proportion of Off-Task behaviors before and after Social Disapproval across all observations, under the expectation that Off-Task behaviors after reinforcers will be less than the proportion before reinforcers. A GEE model was specified with a logit link for the mean and variance of a binomially distributed outcome, with an independence working correlation structure for repeated measures.

The observed proportion of Off-Task behavior before Social Disapproval was .026 and the observed proportion of Off-Task behavior after Social Disapproval was .038 . The difference of .012 between these two proportions was significant using the GEE model $\left(\beta_{1}=.4060\right.$, Wald $\left.\chi^{2}(1)=174, p<.001, S E=.0308\right)$. These findings suggest that Off-Task behavior is $1.2 \%$ more likely to occur after Social Disapproval occurs, 
across all students. This primary analysis directly conflicts with the hypothesis that Social Disapproval punishes Off-Task behaviors, and thus, does not support Hypothesis 3.

Table 22. Research question 2 hypothesis 3 primary analysis

\begin{tabular}{cccc}
\hline Beta & $\begin{array}{c}\text { Coefficient Estimate } \\
\text { (Probability) }\end{array}$ & SE. & Wald $\chi^{2}$ \\
\hline$\beta_{0}$ & -3.778 & .022 & $2,399^{* * *}$ \\
$\beta_{1}$ & .406 & .031 & $174 * * *$ \\
\hline$a . \quad * * *$ indicates $p<.001$ & &
\end{tabular}

Secondary Analyses. A secondary analysis was conducted to test reinforcers using a traditional Allison and Liker (1982) z-test of contingencies, adapted for the analysis of reinforcers. This test compared the probability of Off-Task behaviors following Social Disapproval to the overall base rate probabilities of Off-Task behaviors. The test was performed on the contingency table for recounted frequencies, the average frequencies, and the re-computed frequencies. 
Table 23. Research question 2 hypothesis 3 summary secondary analysis

\begin{tabular}{llccc}
\hline \multirow{2}{*}{$\begin{array}{l}\text { Summary } \\
\text { Series }\end{array}$} & $\begin{array}{l}\text { Reinforcement } \\
\text { Sequence }\end{array}$ & \multicolumn{2}{c}{ Off Task Behavior } & Total \\
\cline { 3 - 4 } Recounted & After & $9,408(.038)$ & $234,793(.961)$ & 244,201 \\
& Before & $8,592(.026)$ & $321,959(.974)$ & 330,551 \\
& Total & 18,000 & 556,752 & 574,752 \\
Average & After & $196(.038)$ & $4,892(.961)$ & 5,088 \\
& Before & $179(.026)$ & $6,707(.974)$ & 6,886 \\
& Total & 375 & 11,599 & 11,974 \\
Recomputed & & & & \\
& After & $194(5088)$ & $4,894(.962)$ & 5,088 \\
& Before & $160(.023)$ & $6,726(.977)$ & 6,886 \\
& Total & 354 & 11,620 & 11,975
\end{tabular}

a. Proportions are computed from the row margins

The Allison and Liker $z$-test (1981) found that the proportion of Off-Task behaviors following punishment was significantly greater than the overall proportion across the recounted observation series $($ diff $=.007, z=28.2, \mathrm{p}<.001)$, the average frequencies $($ diff $=.007, z=4.07, \mathrm{p}<.001)$, and the recounted frequencies $($ diff $=.008, z$ $=4.69, p<.001)$. These secondary analyses do not support the hypothesis that disapproval leads to decreases in Off-Task behaviors. On the contrary, these suggest a reinforcing effect of social disapproval for Off-Task behaviors. Table 24 shows the proportions used in the $\mathrm{z}$ score computations for each of the three contingency tables. 
Table 24. Research question 2 hypothesis 3 proportions

\begin{tabular}{lccccc}
\hline $\begin{array}{l}\text { Summary } \\
\text { Series }\end{array}$ & $\mathrm{p}(\mathrm{XA} \mid \mathrm{X}=$ After $)$ & $\mathrm{p}(\mathrm{XA})$ & $\mathrm{p}(\mathrm{X}=\mathrm{After})$ & $\mathrm{N}$ & $z$ \\
\hline Recounted & .038 & .031 & .425 & 574,752 & 28.2 \\
Average & .038 & .031 & .425 & 11,974 & 4.07 \\
Recomputed & .038 & .030 & .425 & 11,974 & 4.69 \\
\hline
\end{tabular}




\section{Chapter 8}

\section{Discussion}

The current study extends the behavior observational literature by implementing a behavioral change-based measure of social consequences in order to explore whether social consequences affect changes in children's future classroom behaviors. This study relies on two core components: 1) a set of procedures and statistical functions incorporated into a web-application with a graphical user-interface, and 2) an archived set of classroom observations, used in Sage and Kindermann's (1999) assessment of naturally occurring classroom contingencies. These components were synthesized to retest hypothesis on naturally occuring social contingencies to determine whether these putative reinforcers were indeed reinforcing.

The discussion proceeds in three parts. The first part overviews the results of the primary and secondary hypotheses tests and then interprets these major findings in terms of relevant theory. This section extends beyond the realm of strict behaviorism to provide insight from theory gleaned using a contextualistic perspective on children's engagement in classrooms.

The second part of the discussion focuses on the strengths, limitations, and future directions of this study. Particularly, this second part focuses on 1) the reinforcement detection applet (i.e. The Reinforcinator), 2) the recounting procedure and measures, and finally, 3) the implications of non-experimental reinforcement detection on naturalistic observations. The discussion ends by a brief mention of the possible extensions of the planned research and how the reinforcement detection strategy may have uses outside the 
realm of pure observations.

\section{Results overview and implications}

There are two components in testing reinforcers. First, the behavior patterns need to be examined for the direction of change, following a given consequence (i.e. greater or lesser after reinforcement), and secondly, this observed function of a consequence needs to be empirically tested for significance. In this review of the results, I restate the results of each hypothesis test by describing the observed functional relation (i.e. reinforcer or punisher) and whether this was significant by the primary, or secondary, or both, analyses. I also note any discrepancies across analyses (e.g. comparing primary to secondary), and within analyses, such as comparing significance tests in the secondary analyses. The recounting procedure was used to address whether direct Social Approval acted as a reinforcing consequence (i.e., Research Question 1) and whether direct Disapproval acted as a punisher (i.e., Research Question 2).

Research Question 1. Research Question 1 asked whether Social Approval was a reinforcer for On-Task behavior (Hypothesis 1) and Off-Task behavior (Hypothesis 2). Across all observations, Social approval was the second most likely social consequence (behind co-operation), occuring about $2.1 \%$ across all students. For behaviors, On-Task active occurred about $16.3 \%$ of the time and Off-Task active was much less likely, with about a $3.1 \%$ base rate.

Social Approval was shown to significantly increase On-Task behaviors by less than $.05 \%$ and Off-Task behaviors by $1.5 \%$, using generalized estimating equations to account for dependency within students across the recounted observation series. When 
the proportion of On and Off-task behaviors after putative reinforcers were compared to their respective base-rates in the secondary analyses, On-Task behavior was only .03\% greater than base-rate, and Off-task behaviors were $.08 \%$ greater than base-rate proportions. The difference for On-Task behaviors was only significant when using the inflated recounted sample. This contrasts with Off-Task behaviors which were significantly increased after a reinforcing event across the recounted as well as the corrected sample.

The results of research question one brings light to interesting implications for detecting reinforcers under natural conditions. Firstly, these results imply that traditional measures of reinforcers may not uncover the full scope of potential reinforcers. In Sage and Kindermann's (1999) analysis of classroom contingencies, for example, lagsequential analysis revealed that Social Approval was more likely follow On-Task behaviors when compared to chance. This was not the case for Off-Task behaviors. Sage and Kindermann's findings suggested that Social Approval was a reinforcer for On-Task behaviors (and not Off-Task behaviors). The current study finds the opposite, namely, that Off-Task behaviors (and not On-Task behaviors) were more likely to follow Social Approval when compared to chance. Furthermore, the magnitude of change before and after Social Approval was greater for Off-Task behaviors, suggesting that Off-Task behaviors are more sensitive to Social Approval when compared to On-Task behaviors.

Taken as a whole, the discrepancies between the results contingency analysis and reinforcer analysis (when applied to the same set of classroom observations), suggest that there is more to the story of reinforcement then revealed by a single analysis alone. Sage and Kindermann's (1999) contingency analyses show that is more common to observe 
contingent Social Approval for On-Task behaviors, and less common to observe contingent Social Approval for Off-Task behaviors. The reinforcer analysis used in the present study tells us that the effects of Social Approval are greater for Off-Task behaviors than On-Task behaviors. When applied to classroom settings, the findings of the current study serve a cautionary tale; namely, that what looks like reinforcers, may not actually be reinforcing.

The results of Research Question One have a second major implication to the analysis of naturally occurring reinforcers, namely, the overall reinforcing effects may be so small in magnitude that they are barely noticeable in classroom settings, over short periods of time. The increases in On- and Off- Task behaviors from Social Approval were less than $1 \%$ across all observations; much less than what is typically produced from intervention studies (e.g., Soloman \& Wahler, 1973). As noted by Partin and colleagues (2009), typical classroom interventions operate on a much longer timeframe then the twoweek observations used in the current study, which makes a comparison of experimental verse naturally occurring reinforcer effects somewhat tenuous.

A reasonable assumption is that a $1 \%$ increase in behaviors is not noticeable in classrooms, even over the two-week time frame used to collect observations in the current study. This does not imply that the effects of Social Approval stop at $1 \%$ change. To determine a long-term effect of reinforcers, future studies could explore short-term reinforcement patterns with more scrutiny than the overall analysis used in the current study. For example, the reinforcer analysis could look for additive patterns between reinforcers; that is, whether the reinforcer effect increases for subsequent reinforcers. By exploring additive effects, for example, future studies could answer more predictive 
questions, such as how much reinforcement is necessary to make a noticeable change across a timeframe.

Research Question 2. Research Question Two asked whether Social Disapproval has the role of a punisher for children's Off-Task behaviors. Social Disapproval was one of the least frequent social consequences, occurring around $.4 \%$ of the time. Contrary to expectations, Social Disapproval was shown to significantly increase children's Off-Task behavior by approximately $1.2 \%$, using general estimating equations to account for dependency within students. When compared to base-rates in the secondary analyses, Social Disapproval was shown to increase Off-Task behaviors relative to base-rate by about $.07 \%$. These increases in Off-Task behaviors go against the expectation that Disapproval was a punisher.

The unexpected findings of Research Question Two have major technical and theoretical implications. Technically, the increase in Off-Task behaviors following Social Disapproval may be an artifact of previous reinforcement from Social Approval of OffTask behaviors. Specifically, it is possible that Off-Task behaviors were first met by Social Approval and then future Off-Task behaviors were met by Social Disapproval. In this case, the effects of Social Disapproval may be distorted or suppressed by the lingering effects of prior Social Approval. This implies that overall strategy used in current study is limited since it does not control for prior reinforcers in its analysis strategy. This limitation makes it impossible to truly know how Social Approval interacts with Disapproval for Off-Task behaviors. Consequently, this study cannot conclusively say determine whether Disapproval is reinforcing or punishing for Off-Task classroom behaviors. 
Technical limitations aside, the finding that Disapproval increases or sustains OffTask behaviors in classroom settings has precedence outside the strict behaviorist literature. For example, Saxton (2019) used naturalistic observations across four middle school classrooms and found that teacher's disapproval did not resolve approximately $89 \%$ of the interactions with disaffected students. Unlike the current study, these findings drew heavily from the engagement literature; in which student's disaffection is part of a larger multi-faceted and multi-dimensional motivation construct (Skinner et al., 2008). From this perspective, disapproval from adults is at a direct conflict with the emerging adolescent's need for autonomy, and thus, it follows that direct disapproval may 'trigger' future disobedience from students. Taken with the results of Research Question Two in the current study, it seems possible that non-behavioral processes may need to be considered when examining classroom reinforcement patterns.

Outside of classrooms, the reinforcing effects of disapproval do follow from the notion of a 'reinforcement trap'; that is, a feedback loop of negative behaviors, typically studied in childhood aggression and coercive processes in families (e.g., Patterson 1982). The term 'reinforcement trap' in reference to a cycle of escalating aggressive behaviors occuring between two interactants. In these interactions, a child may initiate an aggressive response, only to be met with an aggressive consequence; either physical retaliation or bullying on the playground (e.g., Patterson, 1967), or verbally aggressive, such as a stern direct statement of approval from a parent or teacher. To my knowledge, little research has studied these processes in classroom settings. Taken with the findings of Research Question Two, it is plausible that the observed reinforcement effect was indeed an instance of naturally occurring reinforcement traps. In this case, what looks 
like teacher's use of punishment (e.g. disapproving negative behaviors), actually has the opposite effects on students' future behaviors.

\section{Strengths and Main Contributions of Study}

This study offers a first step forward in the analysis of naturalistic observations with regard to empirically verifiable reinforcement. While plenty of challenges and refinements are expected for the future of this methodology, this study, at the very least, poses a good starting point for addressing limitations of existing methods. This spirit of curiosity led the current explore whether contingency detection really was an 'end all' methodology, or if it could be expanded upon in a way that more accurately sheds light on reinforcement processes in accordance with standard operational definitions and experimental methods. Accordingly, the result was the development of novel procedure for transforming observational datasets, a web-app for distributing the procedure, and a re-analysis of contingency hypotheses to demonstrate the methodology.

This section discusses the strengths, limitations, and future directions of the current study. I organize a discussion around three major pieces: 1) the reinforcement detection strategy, 2) the Reinforcinator web-app, and 3) the use of naturalistic observations. Each topic is introduced in terms of its strengths and is followed by a discussion of its limitations and areas for future improvements.

Reinforcement detection strategy. The reinforcement detection strategy comprises two components: a 'classification' piece and a 'measurement' piece. The analyses of this thesis synthesized these pieces under an 'overall strategy'. In this strategy, the observations are classified by the variable reinforcement sequence (e.g. 
'before' and 'after' reinforcement) as reinforcers occur across all individual students. The relation between the reinforcement sequence and outcome is measured across all students. Ultimately, this synthesis yielded mixed results in terms of hypothesis testing, but also, shed light on many areas for future versions of the program and tangible next steps for future studies.

The 'classification piece' of the reinforcement detection strategy is used to identify observations as occuring by reinforcement sequence (i.e. 'before' or 'after' reinforcers) for behaviors (e.g. 'targets' or 'non-targets'). While other studies have attempted to detect reinforcers under 'naturalistic conditions', this is the first study to our knowledge which provides an intuitive computationally simple and intuitive approach (see Appendix B for a more detailed review of prior attempts at naturalistic reinforcement detection). The 'classification piece' (i.e., recounting procedure) of this strategy produces a versatile dataset which retains all original variables and can be summarized by contingency tables and directly tested in regression models.

The 'measurement piece' of the strategy comprises a regression and contingency table tests for different hypotheses. A general estimating equations approach was used to test the difference in proportions. This approach was useful for testing an 'overall' measure of reinforcers; whereby the parameter estimates were identical to raw proportions from contingency tables but with a standard error which accounts for dependencies in repeated observations within individuals. The interested reader is encouraged to see Appendix $\mathrm{H}$ for a comparison of the GEE models under an ‘exchangeable' working correlation structure, and with naïve standard errors from logistic regression models. 
Current limitations. The measurement piece of the strategy was implemented at an 'overall level of analysis'; meaning, behaviors are identified as 'before' or 'after' a given reinforcer at the level of observation series. This potentially clashes with theories of learning since it treats all observations as coming from one person's observation series, without distinguishing between individuals. For a first step, however, it was important to conduct reinforcement tests from a "within-" individual perspective, so that all behaviors 'before' and 'after' reinforcers were assumed to belong to same subject (see Skinner, 1953 for a comprehensive discussion). Nevertheless, by not calculating reinforcement within individuals, the current analysis potentially misses individual differences with regards to reinforcement patterns.

Figure 30. Individual reinforcement patterns.

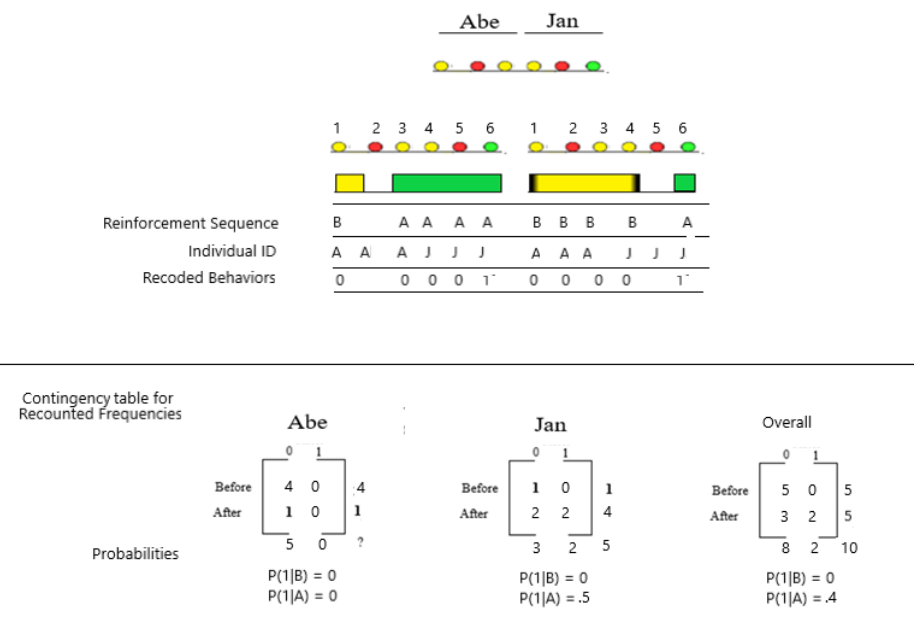

I use Figure 30 to fully illustrate the overall analysis and its limitations regarding individual learning. This figure shows two individuals, with 3 observations. One student (e.g. Abe) has no reinforcement effects and the other student (e.g. Jan) does. This figure shows the $2 \times 2$ contingency table for each student and the probability of On-task 
behaviors after reinforcers. We can average the probabilities across both students to get average probabilities of 0 before and .25 after reinforcers. The third table shows the overall classification of observations, which yields a total probability of .4 after reinforcers. This values in this table are computed by taking the sum across both individuals, and thus, the overall proportion is not an average of individual's behaviors. Consequently, it does not shed light on who is most affected by reinforcers, but instead indicates a reinforcement was present in the observation series.

A second limitation of this study is that the methods do not account for the affects of multiple reinforcers in a single analysis. The ramifications of this limitation were apparent in tests regarding Off-task behaviors (see hypotheses 2 and 3). In these tests, Off-task behaviors were shown to increase following Social Approval and Social Disapproval consequences. This result made it clear that the current strategy is unable to distinguish between effects of a multiple consequences on the same behaviors. Without knowing the general mathematical relations between reinforcers and punishers generally (e.g., how many punishers will negate the effects of reinforcers?), the challenge of parsing out lingering reinforcement effects from 'fresh' punishment effects becomes a very daunting task for naturalistic observations.

Finally, the reinforcement detections strategy is limited from the perspective of criterion validity. The secondary analyses do indeed test reinforcement from a different lens compared to the primary analyses. This lens may be a little too different in that it tests a slightly different hypothesis, using a different set of proportions, for its calculation of reinforcer effects. From a perspective of criterion validity, a more preferential strategy would be to test the same exact hypothesis in two different ways (e.g. regression and 
contingency tables, for example). As discussed in Chapter 5, this study envisioned reinforcement as an extension of lag-sequential analysis. In this way, the current study is limited in that it does not repeat the same test using two different procedures.

A consequence of this limited validity is interpreting the regression models. While the GEE models were a promising start for this study, further comparisons showed that the independence assumption is likely wrong (see Appendix $\mathrm{H}$ for details). Follow up analyses revealed that all models in the primary analysis were less efficient than those under an exchangeable working correlation structure, using the QIC for comparing model fit (Shen \& Chen, 2012). This could be due to the violation of the GEE assumption that no higher order of clustering exists. In the current study, sub-series is the highest order of nesting and supersedes individual identity, thus, violating this assumption of generalized estimating equations, and potentially providing unreliable standard error estimates. Lastly, without an alternative for comparing the same hypotheses, the results of the regression models must be taken cautiously since they use a much larger recounting sample than the original observations, thus, possibly exaggerating the reported effects.

Future directions. Future versions of the recounting procedure address the stated limitations in the following ways. Firstly, future versions are encouraged to account for effects within individuals for each reinforcer at a time. Such models may want to apply the recounting procedure on each individual behavior series for a given reinforcer to obtain an individual recounted behavior series. The effects of a single variable could be assessed by incorporating these individual behavior series into a single data matrix to be used in a mixed effect model for a single outcome (e.g., one reinforcer). In this way, an individual series for Abe and Jan (see Figure 30), would be automatically added on to the 
recounted data-file.

To account for multiple reinforcer effects (and potential conflicts with punishers), future studies are encouraged to apply the recounting procedure across each possible behavior-consequence relation in an observation series. Using the data in this thesis, this would account for 30 different recounted observation series. If this approach was repeated for individuals separately, then a future analysis could comprehensively account for all reinforcement and punishment patterns, within and across individuals. While the formulation of such models is beyond the scope of this study, structural equation models for latent variables are one possible avenue for analyzing such datasets. The challenge for these studies would largely be consolidation and computational efficiency, as such a model would yield so much data that a standard analysis in R may not be feasible for most purposes. In this case, it is encouraged that such a study would also explore combining like codes (e.g., Active and Passive On-Task behaviors), or removing irrelevant codes (e.g. move away from camera).

Finally, future studies are encouraged to address the issue of criterion validity by formally expanding the recounting procedure to explicitly produce a transition table for matched pairs. While Chapter 5 touched upon this topic, it would be necessary to adjust the recounting procedure algorithmically so that transitions are computed for missing pairs, obtained from matching indices for observations before or after reinforcers. By directly computing transitions for matched pairs, even with incomplete pairs, future studies can apply variations of the Mcnemar test for complete, incomplete, and combined tables (see Tang \& Tang, 2003 for such strategies). This is advantageous from the validity perspective; in that it would allow for the same hypothesis to be tested multiple ways. 
This would allow the results of the regression to be verified using the original sample sizes, by performing an Mcnemar test on the average recounted pairs, for example.

Reinforcement Detection Applets. The Reinforcinator applet is a promising start for a software implementation of the reinforcement detection strategy in this thesis. This app contains all the tools used in the analysis of the present study (e.g., recounting procedure, gee models, contingency tables, etc.). Accordingly, it is possible to replicate all the analyses of this study, simply by using the app. Additionally, the app contains selected text and illustrations from this thesis, so that any user can have a basic conceptual understanding of the procedures. Taken as a whole, this is a step towards filling a void in the social developmental and applied behavior analysis literatures; where researchers have been arguing for the necessity of software and methods to extend observational analysis of reinforcers beyond extant methods, such as lag-sequential analysis (e.g. Sloman 2010; Dishion \& Doge, 2005).

Current limitations. Despite its strengths, the applet still has a room for many improvements. One of the main concerns is computational efficiency and stability. To replicate the analysis in this study, for example, it may take the user upwards of 20 minutes to run a single GEE model on the recounting observations! There are two main reasons for this. A first reason has to do with the computational efficiency of the specific functions used to implement the recounting procedure. These can be improved upon using more efficient computing procedures and data structures. The second reason has to do with the R Shiny interface. Since the functions are ran in $\mathrm{R}$ and Shiny, the computational speed is compounded and may also interact with user's web-access (e.g. specific web-browsers and internet connection). 
Future directions. Future version of the Reinforcinator web-app are encouraged to step away from the Shiny platform and R software. Alternative frameworks may be more efficient in other programming languages (e.g., Django and Python). Future revisions are encouraged to implement some of the corrections noted for future versions of the recounting procedure (e.g., iterative recounting across all possible behaviorconsequence combinations; recounting across all individuals; alternative statistical models, etc.). As noted previously, certain additional functions may facilitate these computations and are encouraged for future versions of the program (e.g., combining and removing codes).

Naturalistic Observations. The current study contributes to the literature on peer influence by examining the effects of consequences, independently of contingency levels. By re-examining hypotheses from Sage and Kindermann's (1999) study, the current thesis shows reinforcement effects for Social Approval and Disapproval for student's On and Off-task behaviors. Taken at face value, these findings suggest there is an increase in these student behaviors across the observation series and these increases are related to putative reinforcers. These findings accord with experimental and social development literature on potential reinforcers experienced in classroom settings (e.g., Greenwood, Delquadri \& Hall, 1984; Godwin, Almeda, Petroccia, Baker, \& Fisher, 2013; Vitiello, Booren, Downer, Williford, 2012).

Current limitations. Aside from known issues of the methodology (e.g., inability to control for confounds of prior experience from conflicting reinforcers, different hypothesis tests for all observations and contingency tables, inflated sample size for regression approaches, etc.), the current study is limited on theoretical grounds for not 
investigating contextual effects on social reinforcers. A large part of Sage and Kindermann's study was to compare contingencies across different social partners (e.g., peer group members vs. teachers). The current study does not distinguish these effects. The inability of the current study to clearly distinguish contextual effects from reinforcer effects may be most consequential for the analysis of Off-Task behaviors. Perhaps, if changes in Off-Task behaviors were measured independently for Disapproval from one's peers or from the teachers, the study may find punishing effects for one social context exclusively (e.g. disapproval from teachers as a punisher).

Future revisions. The future of this methodology moves in two primary directions. Firstly, it is important to re-examine these classroom reinforcers once more, under a more refined lens that accounts for multiple reinforcers, social contexts, and individual differences. By implementing the noted changes in the recounting procedure, future studies may be able to more comprehensively comb through Sage and Kindermann's (1999) to further distinguish contexts in which social consequences derive specific reinforcing or punishing functions. Relatedly, future studies are encouraged to also account for the known contingencies when computing reinforcer effects. As noted by Staddon and Cerruti (2003), reinforcement is theoretically a function of contingency and behavior change, such that knowing one without the other can only provide a fragmented picture in the best-case scenario.

A second direction for future revisions is a clear departure from the substantive application of the current study. Generally, the methods presented in this thesis are not relegated to classroom behaviors or social reinforcers. Stated differently, the recounting procedure can be used for any environmental 'event' as a reinforcer for any type of 
behaviors. The only requirement of this strategy is longitudinal observations of the dependent variable. In this way, the strategy can be extended to analyze anything from changes in voting behaviors due to a political change, to company performance measures as stock prices change, to the frequency of elevator usage depending on elevator arrivals. A much-anticipated feature of this study is that it seeing which doors open for the analysis of reinforcer control over any behavior-environment observations. In this way, this thesis is a very promising start to the extension of Skinner's original notion of reinforcers to the analysis of real-world behaviors. 


\section{References}

Agretsi, A. (2013). Categorical Data Analysis. Hoboken: John Wiley and Sons.

Alber, S. R., \& Heward, W. L. (1996). "gotcha!" twenty-five behavior traps guaranteed to extend your students' academic and social skills. Intervention in School and Clinic, 31(5), 285-89

Allison P. D., \& Liker, J.K. (1982). Analyzing sequential categorical data on dyadic Interaction. Psychological Bulletin, 91, 393 - 403.

Altmann, J. (1974). Observational study of behavior: sampling methods. Behaviour, 49(3), 227-266.

American Psychological Association. (2009). Concise rules of APA style. American Psychological Association.

Andrews, L., \& Kozma, A. (1990). Increasing teacher praise and altering its distribution to children of differing on-task levels. Canadian Journal of Behavioural Science/Revue canadienne des sciences du comportement, 22(2), 110.

Ator, N. A. (1999). Statistical inference in behavior analysis: Environmental determinants?. The Behavior Analyst, 22(2), 93.

Azrin, N. H., \& Lindsley, O. R. (1956). The reinforcement of cooperation between children. The Journal of Abnormal and Social Psychology, 52(1), 100

Bakeman, R., \& Gottman, J. M. (1997). Observing interaction: An introduction to sequential analysis. Cambridge university press

Bakeman, R., Deckner, D. F., \& Quera, V. (2005). Analysis of behavioral streams. In D. M. Teti (Ed.), Handbook of research methods in developmental science (pp. 394420). Oxford, UK: Blackwell Publishers

Bakeman, R., \& Quera, V. (1995). Log-linear approaches to lag-sequential analysis when consecutive codes may and cannot repeat. Psychological Bulletin, 118(2), 272.

Bakeman, R., \& Quera, V. (2008). ActSds and OdfSds: Programs for converting INTERACT and The Observer data-files into SDIS timed-event sequential datafiles. Behavior research methods, 40(3), 869-872.

Baker, J. A. (1999). Teacher-student interaction in urban at-risk classrooms: Differential behavior, relationship quality, and student satisfaction with school. The Elementary School Journal, 57-70.

Baltes, M. M. (1988). The etiology and maintenance of dependency in the elderly: Three phases of operant research. Behavior Therapy, 19(3), 301-319. 179.

Baron, R. M. (1966). Social reinforcement effects as a function of social reinforcement history. Psychological Review, 73(6), 527. 
Beaman, R., \& Wheldall, K. (2000). Teachers' use of approval and disapproval in the classroom. Educational Psychology, 20(4), 431-446.

Beavers, G. A., Iwata, B. A., \& Lerman, D. C. (2013). Thirty years of research on the functional analysis of problem behavior. Journal of Applied Behavior Analysis, 46(1), 1-21.

Broden, M., Bruce, C., Mitchell, M. A., Carter, V., \& Hall, R. V. (1970). Effects of teacher attention on attending behavior of two boys at adjacent desks. Journal of Applied Behavior Analysis, 3(3), 205-211.

Brophy, J. (1981). Teacher praise: A functional analysis. Review of educational research,51(1), 5-32.

Burnett, P. C. (2001). Elementary students' preferences for teacher praise. The Journal of Classroom Interaction, 16-23.

Cameron, J., \& Pierce, W. D. (1994). Reinforcement, reward, and intrinsic motivation: A meta-analysis. Review of Educational research, 64(3), 363-423.

Carey, V. J., Lumley, T., \& Ripley, B.D. (2012) gee: Generalized estimation equation solver. URL http//CRAN.R-project.org/package=gee, $R$ package version, 4-13.

Chalk, K., \& Bizo, L. A. (2004). Specific praise improves on-task behaviour and numeracy enjoyment: A study of year four pupils engaged in the numeracy hour. Educational Psychology in Practice, 20(4), 335-351.

Chang, W., Cheng, J., Allaire, JJ., Xie, Y., \& McPherson, J., (2016). Shiny: Web Application Framework for R. R package R package version 0.14.2. https://CRAN.R-project.org/package=shiny

Charlesworth, R., \& Hartup, W. W. (1967). Positive social reinforcement in the nursery school peer group. Child Development, 993-1002.

Choi, S. C., \& Stablein, D. M. (1982). Practical tests for comparing two proportions with incomplete data. Journal of the Royal Statistical Society: Series C (Applied Statistics), 31(3), 256-262.

Dart, E. H., Collins, T. A., Klingbeil, D. A., McKinley, L. E., \& VanDerHeyden, A. (2014). Peer management interventions: A meta-analytic review of single-case research. School Psychology Review, 43(4), 367-384.

Dishion, T. J., Duncan, T. E., Eddy, J. M., Fagot, B. I., \& Fetrow, R. (1994). The world of parents and peers: Coercive exchanges and children's social adaptation. Social Development, 3(3), 255-268.

Dishion, T. J., Spracklen, K. M., Andrews, D. W., \& Patterson, G. R. (1996). Deviancy training in male adolescent friendships. Behavior therapy, 27(3), 373-390.

Dishion, T. J., Piehler, T. F., \& Myers, M. W. (2008). Dynamics and ecology of 
adolescent peer influence.

Elwell, W. C., \& Tiberio, J. (1994). Teacher praise: What students want. Journal of Instructional Psychology, 21(4), 322.

Fagerland, M. W., Lydersen, S., \& Laake, P. (2013). The McNemar test for binary matched-pairs data: mid-p and asymptotic are better than exact conditional. BMC medical research methodology, 13(1), 91.

Farmer, T. W., Dawes, M., Alexander, Q., \& Brooks, D. S. (2016). Challenges Associated With Applications and Interventions: Correlated Constrains, Shadows of Synchrony, and Teacher/Institutional Factors that Impact Social Change. Handbook of Social Influences in School Contexts: Social-Emotional, Motivation, and Cognitive Outcomes, 423.

Ferster, C. B., \& Skinner, B. F. (1957). Schedules of reinforcement. New York: AppeltonCentury-Crofts.

Gallistel, C. R., Craig, A. R., \& Shahan, T. A. (2014). Temporal contingency. Behavioural processes, 101, 89-96.

Gewirtz, J. L., \& Peláez-Nogueras, M. (1992). BF Skinner's legacy in human infant behavior and development. American Psychologist, 47(11), 1411.

Gewirtz, J.L. (1997). The response stimulus contingency and reinforcement learning as a context for considering two non-behavior analytic views of contingency learning. The Behavior Analyst, 20(2), 121-128

Godwin, K. E., Almeda, M. V., Petroccia, M., Baker, R. S., \& Fisher, A. V. (2013). Classroom activities and off-task behavior in elementary school children. Cognitive Science Society.

Gosho, M (2014). Criteria to select a working correlation structure for the generalized estimating equations method in SAS. Journal of Statistical Software, 57(1), 1-10

Gottman, H.M. (1980). On analyzing for sequential connection and assessing interobserver reliability for the sequential analysis of observation data. Behavioral Assessment, 2, 361-368.

Gottman, J. M., \& Krokoff, L. J. (1989). Marital interaction and satisfaction: A longitudinal view. Journal of consulting and clinical psychology, 57(1), 47.

Gottman, J., \& Roy, A. K. (1990). Sequential analysis: a guide for behavorial researchers. Cambridge University Press.

Greenwood, C. R., Delquadri, J., \& Hall, R. V. (1984). Opportunity to respond and student academic performance. Focus on behavior analysis in education, 58-88

Greenwood, C. R., Delquadri, J. C., \& Hall, R. V. (1989). Longitudinal effects of classwide peer tutoring. Journal of educational psychology, 81(3), 371. 
Halekoh, U., Højsgaard, S., \& Yan, J. (2006). The R package geepack for generalized estimating equations. Journal of Statistical Software, 15(2), 1-11.

Hanley, James A., et al. "Statistical analysis of correlated data using generalized estimating equations: an orientation." American journal of epidemiology 157.4 (2003): 364-375

Hay, W. M., Hay, L. R., \& Nelson, R. O. (1977). Direct and collateral changes in on-task and academic behavior resulting from on-task versus academic contingencies. Behavior Therapy, 8(3), 431-441.

Herrnstein, R. J. (1974). Formal properties of the matching law. Journal of the experimental analysis of behavior, 21(1), 159-164.

Hintze, J.M., \& Matthews, W.J. (2004). The generalizability of systematic direct observations across time and setting: A preliminary investigation of the psychometrics of behavioral observation. School Psychology Review, 33, 258-270.

Horner, R. H., Carr, E. G., Halle, J., McGee, G., Odom, S., \& Wolery, M. (2005). The use of single-subject research to identify evidence-based practice in special education. Exceptional Children, 71(2), 165-179.

Horn, W. F., Conners, C. K., Wells, K. C., \& Shaw, D. (1986). Use of the Abikoff classroom observation coding system on a children's inpatient psychiatric unit. Journal of psychopathology and behavioral assessment, 8(1), 9-23.

Heagerty, P. J., \& Zeger, S. L. (1996). Marginal regression models for clustered ordinal measurements. Journal of the American Statistical Association, 91(435), 1024-1036.

Houghton, S., Wheldall, K., Jukes, R. O. D., \& Sharpe, A. (1990). The effects of limited private reprimands and increased private praise on classroom behaviour in four British secondary school classes. British Journal of Educational Psychology, 60(3), 255-265.

Hutchings, J., Martin-Forbes, P., Daley, D., \& Williams, M. E. (2013). A randomized controlled trial of the impact of a teacher classroom management program on the classroom behavior of children with and without behavior problems. Journal of school psychology, 51(5), 571-585.

Hughes, J. N., Luo, W., Kwok, O. M., \& Loyd, L. K. (2008). Teacher-student support, effortful engagement, and achievement: A 3-year longitudinal study. Journal of educational psychology, 100(1), 1.

Johnson, L. M., \& Morris, E. K. (1987). When speaking of probability in behavior analysis. Behaviorism, 107-130.

Kazdin, A. E., \& Klock, J. (1973). The Effect Of Nonverbal Teacher Approval On Student Attentive Behavior. Journal of Applied Behavior Analysis, 6(4), 643-654.

Kerr, M. M., Zigmond, N., Schaeffer, A. L., \& Brown, G. M. (1986). An observational follow-up study of successful and unsuccessful high school students. The High School Journal, 20-24. 
Killeen, P. R. (1994). Mathematical principles of reinforcement. Behavioral and Brain Sciences, 17(01), 105-135.

Kindermann, T., \& Skinner, E. A. (1988). Developmental tasks as organizers of children's ecologies: Mothers' contingencies as children learn to walk, eat, and dress In J. Valsiner (Ed.), hildren's development within socio-culturally structured environments (pp. 66105)., Norwood, N.J: Abex.

Kindermann, T. (2018, September 29). Personal interview.

Kish, L. (1995). Survey sampling (Vol. 60). Wiley-Interscience.

Kleinman, K. E., \& Saigh, P. A. (2011). The effects of the Good Behavior Game on the conduct of regular education New York City high school students. Behavior Modification, 35(1), 95-105.

Jenkins, L. N., Floress, M. T., \& Reinke, W. (2015). Rates and types of teacher praise: A review and future directions. Psychology in the Schools, 52(5), 463-476.

Liang, K.Y., \& Zeger, S.L. (1986). Longitudinal data analyses using generalized linear models. Biometrika, 73(1), 13-22.

Lattal, K. A., \& Poling, A. D. (1981). Describing response-event relations: Babel revisited. The Behavior Analyst, 4(2), 143

Lattal, K. A., \& Abreu Rodrigues, J. (1997). Response Independent Events In The Behavior Stream. Journal of the Experimental Analysis of Behavior, 68(3), 375398

Maccoby, E. E. (2007). Historical overview of socialization research and theory. Handbook of socialization: Theory and research, 13-41.

Martens, B. K., Lochner, D. G., \& Kelly, S. Q. (1992). The effects of variable-interval reinforcement on academic engagement: A demonstration of matching theory. Journal of Applied Behavior Analysis, 25(1), 143-151.

Martens, B. K., Bradley, T. A., \& Eckert, T. L. (1997). Effects of reinforcement history and instructions on the persistence of student engagement. Journal of Applied Behavior Analysis, 30(3), 569-572.

McComas, J. J., Moore, T., Dahl, N., Hartman, E., Hoch, J., \& Symons, F. (2009). Calculating contingencies in natural environments: Issues in the application of sequential analysis. Journal of applied behavior analysis, 42(2), 413-423.

McDowell, J., \& Caron, M. L. (2010). Bias and undermatching in delinquent boys'verbal behavior as a function of their level of deviance. Journal of the experimental analysis of behavior, 93(3), 471-483.

McHugh, M.L. (2013). The chi-square test of independence. Biochemica media: Biochemia medica 23, no.2 2013):143-149. 
Pan, W., \& Connett, J. E. (2002). Selecting the working correlation structure in generalized estimating equations with application to the lung health study. Statistica Sinica, 475-490.

Partin, T. C. M., Robertson, R. E., Maggin, D. M., Oliver, R. M., \& Wehby, J. H. (2009). Using teacher praise and opportunities to respond to promote appropriate student behavior. Preventing School Failure: Alternative Education for Children and Youth, 54(3), 172-178.

Patterson, G., \& Anderson, D. (1964). Peers as social reinforcers. Child Development 951-960.

Patterson, G. R., Littman, R. A., \& Bricker, W. (1967). Assertive behavior in children: A step toward a theory of aggression. Monographs of the society for research in child development, 32(5), iii-43.

Patterson, G. (1974). A basis for identifying stimuli which control behaviors in natural settings. Child Development, 900-911.

Patterson, G. R. (1982). Coercive family process (Vol. 3). Castalia Publishing Company.

Pipkin, C.S.P. \& Vollmer, T.R. (2009). Applied Implications of Reinforcement History Effects. Journal of Applied Behavior Analysis, I42I(1), 83-103.

Putallaz, M., \& Gottman, J. M. (1981). An interactional model of children's entry into peer groups. Child development, 986-994.

Quera, V., \& Bakeman, R. (1998). SDIS-GSEQ 2.0: Software for the analysis of behavior sequences. In Measuring Behavior'98, 2nd International Conference on Methods and Techniques in Behavioral Research (Groningen, The Netherlands, 18-21 August 1998) (pp. 238-239).

R Core Team (2016). R: A language and environment for statistical computing. $\mathrm{R}$ Foundation for Statistical Computing, Vienna, Austria. URL https://www.Rproject.org/.

Ramachandran, K. M., \& Tsokos, C. P. (2014). Mathematical statistics with applications in $R$. Elsevier.

Reitan, T., \& Nielsen, A. (2016). Do not divide count data with count data; a story from pollination ecology with implications beyond. PloS one, 11(2), e0149129.

Robert, C., \& Casella, G. (2009). Introducing Monte Carlo Methods with R. Springer Science \& Business Media.

Rubin, K.H., Bukowski, W.M., \& Parker, J.G. (1998). Peer Interactions, relationships, and groups. Handbook of child Psychology.

Rusby, J. C., Estes, A., \& Dishion, T. (1991). The interpersonal process code (IPC). Unpublished manuscript, Oregon Social Learning Center, Eugene 
Sackett, G. P., Holm, R., Crowley, C., \& Henkins, A. (1979). A FORTRAN program for lag sequential analysis of contingency and cyclicity in behavioral interaction data. Behavior Research Methods \& Instrumentation, 11(3), 366-378.

Sage, N. A., \& Kindermann, T. A. (1999). Peer networks, behavior contingencies, and children's engagement in the classroom. Merrill-Palmer Quarterly (1982-), 143171 .

Sasso, G. M., Reimers, T. M., Cooper, L. J., Wacker, D., Berg, W., Steege, M., ... \& Allaire, A. (1992). Use of descriptive and experimental analyses to identify the functional properties of aberrant behavior in school settings. Journal of Applied Behavior Analysis, 25(4), 809-821.

Saxton, E. A. (2019). Examining the Development and Classroom Dynamics of Student Disaffection Over Multiple Time Periods: Short-term Episodes and Long-term Trajectories (Unpublished doctoral dissertation). Portland State University.

Shadish, W. R. (2014). Analysis and meta-analysis of single-case designs: An introduction. Journal of School Psychology, 52(2), 109-122.

Sheeber, L., Hops, H., Andrews, J., Alpert, T., \& Davis, B. (1998). Interactional processes in families with depressed and non-depressed adolescents: Reinforcement of depressive behavior. Behaviour research and therapy, 36(4), 417-427.

Shen, C. W., \& Chen, Y. H. (2012). Model selection for generalized estimating equations accommodating dropout missingness. Biometrics, 68(4), 1046-1054.

Simonsen, B., Fairbanks, S., Briesch, A., Myers, D., \& Sugai, G. (2008). Evidence-based practices in classroom management: Considerations for research to practice. Education and Treatment of Children, 31(3), 351-380.

Sizemore, O., \& Lattal, K. A. (1977). Dependency, temporal contiguity, and response independent reinforcement. Journal of the Experimental Analysis of Behavior, 27(1), 119-125.

Skinner, B. F. (1953). Science and human behavior. New York: Simon and Schuster.

Skinner, B. F. (1992). 'Superstition in the pigeon. 1948. Journal of experimental psychology. General, 121(3), 273.

Skinner, B. F. (1969). Contingencies of reinforcement. New York: Appleton-CenturyCrofts.

Skinner, E. A., Kindermann, T. A., \& Furrer, C. J. (2008). A motivational perspective on engagement and disaffection: Conceptualization and assessment of children's behavioral and emotional participation in academic activities in the classroom. Educational and Psychological Measurement.

Skinner, E. A., Kindermann, T. A., Roeser, R. W., Smith, C. L., Mashburn, A., \& Steele, J. (2015). Applied Developmental Systems Science: Everything You Always Wanted to Know About Theories, Meta-Theories, Methods, and Interventions but 
Didn't Realize You Needed to Ask. An Advanced Textbook.

Sloman, K. N. (2010). Research trends in descriptive analysis. The Behavior Analyst Today, 11(1), 20.

Smith, J. D., Dishion, T. J., Shaw, D. S., Wilson, M. N., Winter, C. C., \& Patterson, G. R. (2014). Coercive family process and early-onset conduct problems from age 2 to school entry. Development and psychopathology, 26(4pt1), 917-932.

Snyder, J., \& Patterson, G. R. (1986). The effects of consequences on patterns of social interaction: A quasi-experimental approach to reinforcement in natural interaction. Child development, 1257-1268.

Snyder, J., West, L., Stockemer, V., Gibbons, S., \& Almquist-Parks, L. (1996). A social learning model of peer choice in the natural environment. Journal of Applied Developmental Psychology, 17(2), 215-237.

Snyder, J., Horsch, E., \& Childs, J. (1997). Peer relationships of young children: Affiliative choices and the shaping of aggressive behavior. Journal of clinical child psychology, 26(2), 145-156.

Solomon, R. W., \& Wahler, R. G. (1973). Peer Reinforcement Control of Classroom Problem Behavior. Journal of Applied Behavior Analysis, 6(1), 49-56.

Solomon, B. G., Klein, S. A., Hintze, J. M., Cressey, J. M., \& Peller, S. L. (2012). A meta-analysis of school-wide positive behavior support: An exploratory study using single-case synthesis. Psychology in the Schools,49 (2), 105-121.

Staddon, J. E. (1984). Social learning theory and the dynamics of interaction.

Staddon, J. E., \& Cerutti, D. T. (2003). Operant conditioning. Annual review of psychology, 54(1), 115-144.

Strand, P. S. (2000). A modern behavioral perspective on child conduct disorder: Integrating behavioral momentum and matching theory. Clinical Psychology Review, 20(5), 593-615.

Strand, P. S., Wahler, R. G., \& Herring, M. (2001). The impact of behavior-specific and behavior-nonspecific reinforcement on child compliance to mother directives. Behaviour Research and Therapy, 39(9), 1085-1097.

Sutherland, K. S., Wehby, J. H., \& Copeland, S. R. (2000). Effect of varying rates of behavior-specific praise on the on-task behavior of students with EBD. Journal of Emotional and Behavioral Disorders, 8(1), 2-8.

Tal,Z. \& Babad.E. (1999). The "teacher's pet" phenomenon as viewed by Israeli teachers and students. The Elementary School Journal, 90(1), 97-108.

Team, R. C. (1999). Writing R extensions.

Tiktin, S., \& Hartup, W. W. (1965). Sociometric status and the reinforcing effectiveness 
of children's peers. Journal of experimental child Psychology, 2(3), 306-315.

Vitiello, V. E., Booren, L. M., Downer, J. T., \& Williford, A. P. (2012). Variation in children's classroom engagement throughout a day in preschool: Relations to classroom and child factors. Early Childhood Research Quarterly, 27(2), 210220.

Vollmer, T. R., \& Hackenberg, T.D. (2001). Reinforcement contingencies and social reinforcement: some reciprocal relations between basic and applied research. Journal of Applied Behavior Analysis, 34(2), 241-253.

Wang, M. (2014). Generalized estimating equations in longitudinal data analysis: a review and recent developments. Advances in Statistics, 2014.

Watson, J. S. (1997). Contingency and its two indices within conditional probability analysis. The Behavior Analyst, 20(2), 129.

Westfall, P. H., Troendle, J. F., \& Pennello, G. (2010). Multiple mcnemar tests. Biometrics, 66(4), 1185-1191.

Wheldall, K., Bevan, K., \& Shortall, K. (1986). A touch of reinforcement: the effects of contingent teacher touch on the classroom behaviour of young children. Educational Review, 38(3), 207-216.

White, R. L. (2010). Demonstration of the effects of an increased praise ratio on student on-task behavior (Doctoral dissertation, University of Kansas).

Zhang, S., Cao, J., \& Ahn, C. (2017). Inference and sample size calculation for clinical trials with incomplete observations of paired binary outcomes. Statistics in medicine, 36(4), 581-591. 


\section{Appendix A}

\section{Experimental Demonstrations of Social Reinforcers in Classrooms}

The only way to tell whether a given event is reinforcing to a given organism under given conditions is to make a direct test. We observe the frequency of a selected response, then make an event contingent upon it and observe any change in frequency. If there is a change, we classify the event as reinforcing to the organism under the existing conditions (Skinner, 1953, p. 122)

Skinner's statement summarizes the basic experimental premise underlying a traditional analysis of reinforcers; that is, a comparison of behavior when its consequences are experimentally controlled (See Chapter 2 for details). Used in laboratory and real-world settings, a traditional analysis of reinforcers has led to a robust body of knowledge regarding the reinforcement of behaviors under ' given conditions'. In this sense, the majority of findings about reinforcers have received the most attention for situations in which reinforcers can be used to modify behaviors and environments (Dixon, Vogel, \& Tarbox, 2012). This contrasts with the purpose of many naturalistic observations, which serve the purpose of explaining existing conditions and behaviors. It is for this reason that I do not include a thorough discussion of traditional reinforcement studies, results, and corresponding theory, in the main body of this thesis.

The proposed reinforcement detection applet does provide a tool for testing whether or not a category of events has a functional effect on behaviors as they occur in the stream of naturalistic observations. Because the reinforcer analyses depends on the quality of the observational datasets, a test of reinforcers may or may not overlook specific conditions which mediate or moderate the functional relations between a particular event and a given behavior. This all depends on whether or not such conditions are explicitly coded (or accounted for) in the observational design (see pages 17-22).

In the case of social events, experimental studies have shown that the delivery (i.e. verbal properties, non-verbal properties, affective properties) as well as the contexts (i.e. group or dyadic interactions, public or private) of social interactions can mediate whether or not a given behavior is reinforcing. Thus, to identify whether or not a particular feature of social approval enhances its reinforcing effects, one would need to account for this variable when coding behaviors and social interactions. As described by Vollmer and Hackenberg (2001):

Unlike tangible reinforcers and punishers, which can be standardized and delivered in a fairly uniform fashion, social consequences come in a variety of forms-facial expressions, physical contact, vocalization - and are delivered in a variety of ways. A major challenge then lies in identifying the features that contribute to the effectiveness of social consequences (p. 248).

When these features are not controlled for in a set of observations, then a test of whether or not a behavior is reinforcing, may overlook critical aspects of what makes a behavior reinforcing. Consequently, such analyses may be less precise than an analyses which accounts for mediators of reinforcers which have support from experimental studies.

The current study explores whether classroom interactions are reinforcing across a wide range of situations, activities, and social partners. In this way, the analyses may combine cases in which social approval is expected to reinforce, with observations in which social approval is not delivered under reinforcing conditions. As a first step in measuring naturally occurring reinforcement patterns, the current thesis does not directly account for the sensitivity of its measure to these potential moderating conditions. Accordingly, I do not discuss these features of social interactions which may produce varying degrees of behavior change, in the main body of this thesis.

This appendix provides a brief overview of the experimental findings regarding those conditions which may or may not moderate the reinforcing effects of Social Approval for On-Task behaviors (i.e. Hypothesis 1), Off-Task behaviors (i.e. Hypotheses 2), as well as the potential punishing effects of Disapproval for Off-Task behaviors (i.e. Hypotheses 3). I selected three potential moderating features of 
classroom consequences on classroom behaviors: 1) its verbal specificity vs generality (i.e. great sentence, vs. good job), 2) whether it is delivered in a public or private setting, that is, in front of other students or during one-on-one interactions, and 3) whether it is delivered by teachers or peers. These conditions were selected because they are not controlled for in the current analyses but are likely to vary across observations of potentially reinforcing interactions, in naturally occurring classroom observations.

I present each topic with a research question that binds the reviewed studies and re-direct the reader to additional literature. I begin my discussion of selected studies by presenting background theory and the rationale behind its research questions and hypotheses. I follow this by discussing the methodological details (i.e. sample, experimental / observational design), and finally, the results and findings of the given study. I conclude my review by discussing how each study supports a given hypothesis and suggestions on how future studies can incorporate these findings when conducting a naturalistic reinforcer analyses, using the proposed reinforcer applet.

\section{Reinforcer Specificity and Academic Behaviors}

Should a teacher verbally describe in detail the On-Task behavior they want to reinforce (i.e. great job raising your hand!), or is it enough to simply express positive affirmations without such details (i.e. good job!). Whether or not verbal specificity adds to the effects of positive affirmations is a question that has received a lot of attention in the behavioral management literature (where teacher approval is often referred to as praise), simply because its answer may be useful for teacher's who wish to maximize the effects of social reinforcers, with the least amount of additional efforts as possible. As teacher's attention and time is a valuable classroom resource, researchers have also asked whether or not teacher's approval of academic performance can generalize to related classroom efforts (i.e. On-Task behaviors).

While the research on teacher's praise has a long history and covers a wide range of topics, this thesis focuses on praise as a direct response of social approval or social affirmation (See Table 2 for examples). The interested reader is re-directed to Kamins and Dweck (1999) for a study on the implications of praise centered on the student (and not behavioral process), as well as Cameron and Pierce (1994) and Deci and Ryan (1999) for early and controversial meta-analytic reviews regarding the effects of praise on student's educational motivation.

The following few paragraphs discuss two studies which show that the social approval is most effective when the student is given specific praise, that is, told what is earning approval (i.e. Chalk \& Bizo, 2004) and that this type of contingency can lead to increases in general On-Task behaviors, when it is made contingent specifically on learning activities and/or academic performance, as opposed to general on-task behaviors (Hay \& Hay, 1977). I selected these studies because the dataset used to test this thesis' hypothesis, uses consequence codes which include both forms of praise, that is, behavior specific and positive praise (so long as it is a direct consequence of behavior) in its operationalization of social approval (see Table 1).

Chalk and Bizo (2004). The purpose of Chalk and Bizo's (2004) was to test the hypothesis that praise is most effective when a teacher describes the behavior earning praise - a type of approval called behavior specific praise. The premise is simply that children will be more likely to re-engage previously reinforced behaviors if they know exactly which behaviors produce the reinforcer. This contrasts with behaviors reinforced by positive praise - a term used in this study to refer to positive and direct social affirmations which do not describe exactly what a student did to earn praise. While this study tested the hypothesis that specific praise is most effective for increasing measures of self-concept and enjoyment of mathematics, in addition to academic behaviors, I focus my description on the effects of praise for On-Task behaviors.

Setting and Sample. The sample was comprised of 109 fourth graders and four classroom teachers from two schools (matched along variables such as size, geographical area, and average family income). All observations took place during the mathematics class.

Experimental Design and Observations. Two teachers were randomly assigned to either a positive or specific praise condition - prior to a 45-minute debriefing. Both conditions required that praise be directed the behavior of either specific children or groups. Teacher's in the behavior specific praise condition were instructed to link praise to a specific rule (i.e. mathematical strategy), effort (i.e. attempt at a challenging problem), or social behavior (i.e. encouraging or assisting a classmate). Teachers in the positive praise condition were instructed to praise behaviors when they occurred but were not given any additional 
instructions. Teachers were required to complete a tally sheet after every lesson - describing the type of praise and behavior. The teacher's agreed to deliver praise according to their assigned conditions, for the approximate sixth month duration of the study.

Observations were conducted across the first half of the school year (from end of summer to end of winter), at three time points. All observations were made during the second half of the numeracy hour when students were instructed to work on assignments based on the lecture from the first 30 minutes of class. The researcher's used the OPTIC coding system for teacher and student behaviors (Merret \& Wheldall, 1986). The OPTIC requires observers to divide a classroom into three even sections and to focus on each group for one minute. The observer focuses on each student within a group for four seconds and records whether or not the child is on or off task. After cycling through each student - the observer then alternates to a new group. Using this procedure, each observation session lasted for approximately 25 to 30 minutes.

Results. Across both conditions, student's rates of on-task behavior increased from baseline to the first observation point and then showed different trajectories towards the final observations; where students in the specific praise condition showed a slight decrease, and those in the general praise condition showed a slight increase in their average percentages of on-task behaviors. Praised produced an increase from baseline to the end of the study, in on-task behaviors across both conditions, with specific praise group showing the largest effects. A repeated-measure analysis of variance performed on baseline and final observations found a significant main effect for change in on-task behaviors $(F(1,2)=41.68, \mathrm{p}<.05)$ but not a significant interaction between conditions $(F(1,2)=.64, \mathrm{p}>.05)$. A second series of between participant effects revealed a significant difference in on-task behavior between conditions $(F(1,2)=41.68$, $\mathrm{p}<.01$ ), such that increases in on-task behavior levels were only significant for those in the specific praise condition.

Hypotheses Support and Future Directions. Chalk and Bizo's study demonstrates that an increase in the frequency of teacher praise leads to increases in On-task behaviors, but that more specific praise may lead to larger effects than positive praise alone. As this thesis does not differentiate between these forms of praise, Chalk and Bizo's findings lend support for the first primary hypothesis. The effects of specific praise may also be present in the dataset used in this thesis' analysis. As an example, an Active On-Task student may add a comment to the teacher's lecture, and she may respond with specific praise by mentioning how the student's comment connects to other aspects of the materials. Alternatively, she may simply smile and say 'thank you for that nice thought'. Chalk and Bizo's findings suggest that either teacher response may reinforce the student's future contributions, but that the former more specific form of praise may lead to greater increases in such behaviors.

Chalk and Bizo's findings lead to potential future analyses of naturally occurring classroom reinforcers. As the current analyses does not differentiate between forms of praise, it may be worth researcher's attention to re-code classroom observations so to distinguish between specific and positive approvals. As a follow-up to the current study, such a recoded analysis could explore whether analytical support for hypothesis 1 depends on the combination of both forms of approval - or whether positive or specific praise alone is enough to show reinforcement effects. Finally, such an analysis could also probe research question 3 , that is, to see whether positive or specific praise depend on the type of On-task behaviors. As Chalk and Bizo's study did not differentiate between active and passive forms, such an analysis may shed light towards a more nuanced understanding of how the specificity of social approval may differentially effects active or passive forms of student's behaviors, in naturally occurring real-world interactions.

Hay's, Nelson, and Hay's (1977). The purpose of Hay's, Nelson and Hay's (1977) study was to assess whether praise for one type of classroom behaviors can lead to generalized reinforcement effects in other types of classroom behaviors. Specifically, it was hypothesized that praise for academic performance (i.e. test results, writing samples) will lead to an increase in performance as well as general on-task efforts (i.e. following instructions, making overt efforts), and that praise for on-task behaviors will not lead to increases in academic performance. These hypotheses followed a simple rationale, namely, that on-task behavior is a necessary, but not sufficient, pre-requisite for academic performance, and thus reinforcing academic performance will lead to concomitant increases in its necessary antecedent (i.e. On-task behavior), but that reinforcing On-task behavior will not necessitate improved performance.

Sample and Setting. The study took place in a small rural elementary school serving a lower 
socioeconomic status community. All observations were conducted during a 30-minute work period for either mathematics or writing activities. Four teachers (one second grade, two third grade, and one fourth grade) were selected to implement each experimental phase. The student sample comprised a total of ten students ( 5 third grade boys, two fourth grade boys, and three second grade boys), who were nominated by their teacher's as the most 'problematic' boys in their classrooms.

Experimental Design and Observations. This study assessed student behavior across three conditions defined by the teacher's use of social approval contingencies. During the baseline phase, teachers were not given any instructions on which behaviors to praise, or how to deliver social approval to their students. For both experimental phases, teachers were instructed to hand each target student a 'good work card', and to mark each card with a star for every time they earned praise. Additionally, teachers were instructed to praise each target student five times during every 30 minute session; where praise statements were defined as any comment that was addressed to the student (by name) and specifically mentioned the behavior or performance earning praise.

A cross-over within subject's strategy, with a reversal sequence, was used to assess behavioral levels across each experimental phase. Five students were randomly assigned to the 'on-task contingency sequence', in which teacher's implemented a baseline, followed by the on-task contingency, return to baseline, and academic contingency phase. The remaining five students were assigned to the 'academic contingency sequence', in which the baseline phases were followed by the academic contingency, and behavioral contingency, to control for the order in which children were exposed to the experimental phase. Each experimental condition lasted for 30 minute daily sessions across seven consecutive school days (28 days total).

All observations were conducted by two trained undergraduate psychology students. A focal observation strategy was used to focus on one child's behavior for five minutes at a time. Each child was observed twice per day, and the order of observations were randomized daily. An interval coding procedure was used to divide each observation episode into 10 -second intervals. The observer would select one instance in the interval to code whether the child was on or off task at that given moment within the tensecond window. On-Task behavior was defined as following teacher instructions, and include all behaviors under the Passive and Active On-task codes used in this thesis (see Table 1). Off-task behaviors were defined as any behavior that was not pertinent to a given activity/instruction (i.e. out of seat or talking to peers).

Results. On-task behavior served as the dependent variable and was measured as the daily percent of intervals containing on-task behaviors. The results were presented by the sequence in which children were presented with the experimental contingencies. In the on-task contingency sequence, the exposure to on-task contingencies led to a $25.4 \%$ increase in student's on-task behaviors compared to baseline, and no change in academic performance. For children in this sequence, the exposure to academic contingencies led to an additional $6.4 \%$ increase in on-task behaviors, that is, a $31.8 \%$ increase from baseline, and also, an increase in the rate and accuracy of academic performances, by 41.9 and 34\%, respectively. Children in the academic contingency sequence, who were first exposed to academic and then on-task contingencies, increased their on-task behaviors by $31.8 \%$ from baseline, when teachers praised their academic performance. The transition to on-task contingencies maintained these percentages in academic performance and accuracy, and increased on task behaviors by an additional $4.9 \%$ (e.g. 36.7\% increase from baseline). Across both sequences, a repeated measure ANOVA (with arc sin transformation for percentage data) found significant effects for the changes in On-task behaviors across both sequences (i.e. $F(3,12)=96.8, \mathrm{p}<.01 ; \mathrm{F}(3,12)=107.7, \mathrm{p}<.01))$, as well as changes in measures of academic performance (i.e. rate of attempting new problems, accuracy of response).

Hypotheses Support and Future Directions. Hay's and colleague's findings lend direct support for the claim that social approval can reinforce students On-Task behaviors. More importantly, however, may be the finding that social reinforcing effects of praise can also effect direct outcomes of On-Task behaviors, namely, student performance. While the current thesis does not examine student performance, this study does measure performance behaviors (i.e. Passive On-Task behaviors). Using an example from this thesis, a student who is actively writing and latter given praise for their assignments, may be more likely to engage in such passive On-Task behaviors in the future, in order to produce similar academic performances, as suggested by Hay's and colleagues findings. In this way, Hay's and colleagues study not only supports the primary hypothesis 1 , but also, supports the expectation that approval will similarly effect 
a combined class of active and passive On-Task behaviors, as explored in this study by research question four.

While the current thesis focuses on direct behavioral changes as evidence for reinforcing effects, future studies are encouraged to consider non-behavioral covariates in order to address the link between student classroom behaviors, the experience of reinforcing consequences, and change in performance related outcomes. One such possible variable may be measures of student's motivation or engagement in the classroom (Skinner, Kindermann, \& Furrer, 2008). This construct embodies a student's overall absorption in the endeavor of schooling and has predictive relations with student achievement (e.g., Hughes, Luo, Kwok, \& Loyd, 2008 ; Vollet, Kindermann, \& Skinner, 2017). By incorporating either engagement or a direct measure of student performance, future studies may use the reinforcement applet to explore whether real-world reinforcement leads to not only direct gains in classroom behaviors, but also, gains in measures of learning, academic performance, and involvement in their schooling careers.

\section{Public or One-On-One Praise}

Can praising a student in front of their entire class counteract the reinforcing effects of praise by bringing unwanted attention to student? Various researchers have suggested that such public displays of teacher's approval may lead to feelings of embarrassment or lead to the image of the 'teacher's pet' (e.g., Bartholomew, 1993; Tal \& Babad, 1989). This may be an important consideration when assessing the reinforcing status of teacher praise - as educational researchers have suggested that praise is only reinforcing so long as it is desired by student's (e.g. Ware, 1978; Brophy, 1981, p. 12). It is to this point that studies have shown that elementary and middle-school aged students largely prefer praise to be delivered privately than publically (Burnett, 2001; Elwell \& Triberio, 1994).

To my knowledge, no study has directly tested whether the context (i.e. public or private) of teacher's social approval alters the functional relation between praise and on-task behaviors - regardless of student's preferences for praise. The following few paragraphs describe a 2014 study by Blaze and colleagues which comes closest to manipulating the privacy of teacher praise, by manipulating the volume of praise and comparing the reinforcement effects of praise heard by multiple students (i.e. loud praise) versus praise heard by only by the student for whom it was intended (i.e. quiet praise). I selected this study because the dataset used in this thesis contains instances of praise delivered in various social settings, and at various volumes. By using a representative sample, the findings from this study are applicable to the claim that social approval is a positive reinforcer under conditions which are likely to occur in naturalistic observations.

Blaze et al. (2012). The purpose of Blaze and colleagues (2012) study was to test whether or not praise functions as a reinforcer for on-task behaviors in public school settings, and whether or not these reinforcer effects depend on praise which complies with prior recommendations. Specifically, it was tested whether or not the effects of praise differ according to whether it is heard by students other than its intended recipients. Thus, this study manipulated the volume of praise - as student's differ along the lines of their preference for private (i.e. low volume) or public (i.e. high volume) delivery, and because student's preference for praise has been espoused a critical variable in the reinforcing effects of teachers use of praise. While the study measured change in On and Off task behaviors, I focus my discussion on results regarding a combined class of Active and Passive On task behaviors.

Setting and Sample. The study took place in four high school classrooms from a school district serving a low socio-economic status community in the southeastern United States. Four teacher's mixed aged classrooms participated - with a total of 83 students across the ninth $(n=62)$, tenth $(n=20)$, and eleventh $(\mathrm{n}=1)$ grade levels. Three of the teacher's taught English and one teacher taught remedial mathematics. This sample was selected on the basis of meeting the criteria that a) the teacher was not currently using a class-wide behavioral intervention and b) that appropriate student behavior occurred in less than $70 \%$ of all observation intervals, in a 20 -minute school wide pre-screening observation phase. All phases of the experiment took place in student' regularly scheduled classes.

Experimental Design and Observations. This study focused on Appropriately Engaged Behavior (AEB) as a dependent variable, where AEB included passive (i.e. reading, writing) and active (i.e. question asking, speaking publically) forms of On-Task behaviors used in this thesis. Students were exposed to three conditions of teachers praise: 1) a baseline phase, in which the teacher was not given any instructions on 
how to deliver praise, 2) a loud phase, in which teachers were instructed to move near the given student and affirm their behavior loud enough for all children to hear, and finally, 3) a quiet phase, in which the teacher was instructed to move near a given student and praise them directly, at a volume which no other students could hear. Across both experimental conditions, praise was to be made on individual students and to occur immediately following a given behavior with a brief description as to why the behavior is receiving praise. All teacher's attended a pre-experiment training session on delivering such behavior specific praise, with role-playing sessions on delivering quiet and loud praise across a variety of classroom conditions.

A multiple baseline within-subjects reversal design was used to expose all students to the same experimental conditions. Two classes were randomly selected to the sequence of A (baseline)-B (loud praise)-C (quiet praise)-A-B. The other two classes were assigned to the sequence A-C-B-A-B. The length of a condition varied across teacher's and the transition from one phase to another was to occur when either a) average AEB exhibited less than $15 \%$ variability across three consecutive days or b) a decreasing trend in AEB. In order to standardize the rate at which teacher's delivered praise, all teacher's provided praise to one student at a rate of one praise statement per two minutes. Each teacher wore an electronic MotivAider device, which provided an electronic signal for delivering praise.

All twenty-minute observation sessions were made by trained graduate students, for each of 22 days of the study. A momentary time sampling procedure was combined with an interval sampling method (See page 19 of this thesis) to assess whether a given interval contained AEB or off-task behaviors. Prior to each 30-minute observation episode, the students were organized into equally sized small groups. The observer sampled one student from each group for two seconds and recorded their behavior as on or off task - as a measure for the entire ten second interval, and then moved to a student in the next group. This process was repeated daily with a randomized order of selecting groups, for each of the 22 days of the study. Observations were conducted across a variety of learning activities (i.e. individual or group projects, exams, lecture, etc.)

Results. On-task behavior was measured as the daily percentage of 10 -second intervals containing on-task behaviors. Across all teacher's classrooms, loud praise produced a $38.9 \%$ increase in average percent of behaviors at baseline (i.e. $49.81 \%$ ). The quiet praise condition lead to a $40.7 \%$ increase from baseline. Both of these effects were partially reversible, as a withdrawal from contingency lead to an average decrease of $21.9 \%$ in treatment effects for on-task behaviors.

In order to account for variation due to schools, a multi-level model was used to assess the average percent of intervals that AEB varied in a) baseline conditions (combining the baseline and withdrawal phase), b) treatment conditions (combining both loud and quiet phases), and c) loud versus quiet phases. A comparison between baseline and treatment conditions yielded a statistically significant effect $(F(1,2.9)=$ $51.8, \mathrm{p}<.01)$ ) for AEB, but such an effect was not found to differ between the two treatment conditions $(F(1,2.9=.043, \mathrm{p}=.043)$. The standardized mean difference effect size for AEB across both treatments was 2.2, suggesting that AEB increases by more than two standard deviations when combining both treatment phases, in comparison to both non-treatment phases. The authors did not report such an effect size for the either distinct treatment phase.

Hypotheses Support and Future Directions. Blaze and Colleagues study make a strong case for the reinforcing effects of teacher praise, irrespective of its 'collateral' audience. As Sage and Kindermann's observational dataset samples across a variety of classroom scenarios and learning activities, it is not unlikely that this thesis assess social approval when delivered in front of groups of students and during private one-on-one interactions. As an example, students who are working on adjacent computer stations may receive direct teacher approval for their projects. Blaze and colleagues findings suggest that this may still exhibit reinforcing effects on future behaviors, even in cases where this interaction is observed by neighboring classmates. This scenario could be interchanged with nearly any class activity, and still likely produce the same results, according to the findings from Blaze and colleagues.

While Blaze and colleagues study suggests that the publicity of a social interaction does not make a functional difference in the reinforcing effects of praise, it does so at a very general level of analyses, that is, by speaking to teacher-student interactions, and across students of various motivational orientations towards their schooling. As many researchers are interested in how to increase academic involvement in students who are less likely to participate in class activities, future studies are encouraged to take a closer look at whether the effects of teacher praise vary for these sub-groups, when praise is delivered in front of their peers or in private interactions. As student's tend to affiliate according to their levels of academic 
engagement (e.g. Kindermann, 2007), such an analysis may be informative as to whether or not the publicity of praise has different effects for those who are, or are not, already engaged by the endeavor of schooling, and thus, likely to have different preferences for praise then their motivational counterparts.

\section{Peer Delivered Reinforcers and Punishers}

Are the reinforcing effects of social approval exclusive to teachers, or can approval from one's classmates also reinforce behaviors? The research area of peer influence has received substantial attention across the past few decades, with research showing the unique contribution of peers towards adolescent's social and psychological development (See Brown \& Larson, 2009 for a review). Of particular interest to developmental researchers are the unique contributions of an adolescent's 'self-selected' group of peers, that is, those children whom an adolescent chooses to spend their time with (i.e. friends, groups of shared interest, etc.). This line of research has shown that self-selected peers can contribute to the development of school related behaviors, such as engagement in school (e.g. Kindermann, 2007), as well as rule-violations and deviant behaviors (e.g. Dishion et al., 1996). While the role of social reinforcement has been implied as a mechanism by which self-selected peers exert influence, studies have mainly examined behaviorconsequence contingency patterns, and have not looked at how social consequences functionally effect future behavior patterns in naturally occurring social interactions between self-selected peers.

Since the early 1970's, behaviorists have tested whether or not peers consequences can be reinforcing for On and Off task classroom behaviors. Unlike the developmentally oriented studies, this line of research has looked at the reinforcing role of age mates, and not necessarily self-selected peer affiliates (see McGee, Kaufmann, \& Nussen, 1977 for an early review). For the purposes of controlling the behaviors of their students, teachers could directly benefit from utilizing peer delivered reinforcers and punishers, as a way to control the periphery of their classrooms. It is in this way that many peer-based behavioral management interventions have sprung up to encourage classmates to enforce appropriate behaviors, whilst being rewarded themselves by access to special privileges (i.e. prizes) or group rewards (i.e. early recess). The interested reader is re-directed to Broussard \& Northrup (1997) and Dart and colleagues (2014) for reviews of contemporary peer-based class-wide behavioral interventions.

Solomon and Whaler's (1973) seminal study, was one of the first to demonstrate that classmates can control each other's On and Off-task behaviors, in typical classroom settings, by mechanisms of Social Approval and Disapproval for On and Off-Task behaviors. I selected this study because it supports the test of each primary hypothesis, by showing that peers can increase each other's On or Off task behaviors by the consequences they provide. As the hypotheses of this thesis are tested across all social partners (and affiliations between classmates), Solomon and Whaler's findings are relevant enough to warrant a claim that social approval and disapproval can reinforce/punish active forms of On and Off task behaviors.

Solomon and Whaler (1973). The purpose of Solomon and Whaler's (1973) study was to determine whether or not one's peer classmates can control each other's behaviors as a function of the consequences they provide. Specifically, this study asked whether or not peer social consequences can contribute to the initial maintenance of deviant classroom behaviors, and whether or not adults can control the social reinforcement of peers in order to produce improvements in appropriate classroom behaviors. These questions were based off earlier naturalistic studies, which demonstrated peer reinforcement of deviant behaviors from social consequences (e.g. Patterson, Littman, \& Bricker, 1967), and also, classroom experiments which demonstrated peer reinforcement of positive behaviors, where peers delivered nonsocial reinforcers, such as good behavior points (e.g. Patterson \& Broddsky,1966). Unlike prior studies at this time, Solomon and Whaler's study focused on both functions of peer reinforcers, that is, for On and Off task behaviors, and specifically, whether these functions can be manipulated by adults to achieve an classroom climate more conducive to learning.

Setting and Sample. This study took place during a social studies period in a single sixth grade classroom in a rural county school. The classroom comprised one teacher and thirty students; however, the intervention focused on a sample of ten children who were nominated by their teacher as the least wellbehaved pupils. Five children were selected as confederates, or 'peer therapists'. These children were selected based on results of a sociometric survey (administered to all students) as well as teacher nominations for which children are most likely to comply.

Experimental Design and Observations. The experiment consisted of a baseline and treatment 
phase, where both phases were replicated once in an $A B A B$ within subject design. At the beginning of the study, the classroom seating arrangements were modified so that one 'peer therapist' was seated adjacent to one target subject. Following baseline observations, the peer therapists were instructed to differentially reinforce on-task behaviors while discouraging off-task behaviors. Rather than exclusively expressing direct approval or disapproval, these peer therapists were told to attend to (i.e. look at or express direct approval) On-Task behaviors and to ignore (i.e. look away from or express direct Disapproval). In this study, Off-Task behavior was defined by any breach of the rules during social studies, and On-Task behavior was defined by compliance of these rules.

Observations were conducted across the duration of the study (i.e. 46 consecutive school days) during the same social studies period. A focal person observation strategy was used to continuously record each focal student's behavior for 6 minutes each day. Each six-minute interval was divided into 360 ten second intervals, observers were instructed to record the first On or Off task behavior falling within each interval. Additionally, observers were to mark any occurrence of social approval or disapproval from either the teacher or the peer therapists. Observations were conducted by two trained observers were stationed at the back of the room and began each observation with a randomly assigned focal student - and shifted to the next student in a sequence that was randomized daily. The baseline phase of the study began after a prescreening period - where observations lasted until both observers met an $80 \%$ agreement rate on all focal student and social partner behaviors.

Results. Off-task behaviors served as the dependent variable and was measured as the percent of intervals containing off-task behaviors, for each child, across all days for each experimental phase ${ }^{9}$. A descriptive analysis on individual children demonstrated a clear pattern across the phases; where each student decreased their Off-task behaviors when ignored by peers from baseline, with a difference of $37.2 \%$ averaged across children. This pattern was replicated in the second phase of the study (i.e. baseline 2 and treatment 2), with an average $37 \%$ decrease. Furthermore, children's Off-Task behaviors dropped from the first to second baselines, with an average 13\% decrease, and, a 12.8 decrease from the first to second treatment phases.

A second set of analyses was presented in order to show the percentages of social partner attention paid to On and Off task behaviors, across all phases of the study. During the baseline phase, only the teacher paid attention to target student's On-task behaviors, and peers attended exclusively to off-task behaviors. This pattern switched during the first treatment phase, as the target student's reduced their offtask behaviors, the control peers became the biggest supports of their on-task behaviors, and gave less attention to Off-task behaviors than the teacher or other classmates. During the second baseline phase, where off-task behavior increased from the prior treatment phase, the teacher was once again the sole source of support or On-task behaviors, where the peer therapists and other classmates almost exclusively attended to Off-task behaviors. Finally, during the last treatment phase, peer therapists and the teacher were the only sources of support for On-task behaviors. During this phase, in which Off-task behaviors were lowest during the study, peer therapists provided the least amount of attention to off-task behaviors. While this analysis did not explicitly correlate attention with changes in student behaviors, these trends were used to infer that peers can control changes in on and off-task behaviors, and that approval for positive behaviors from certain peers can counteract attention for negative behaviors from other peers, over and above supports provided by the teacher.

Hypotheses Support and Future Directions. Solomon and Whaler's study provides further support for the claim that social approval can reinforce On-Task behaviors. Additionally, this study lends support to the second and third primary hypotheses of this thesis, that social approval can reinforce OffTask behaviors, and, that disapproval can punish such behaviors. Regarding these latter results it is of particular interest that peers were the most likely source of naturally occurring reinforcement for Off-Task behaviors, and that disapproval for Off-Task behaviors was only likely from certain peers under adult instructions. As the analyses in this thesis do not differentiate peer from teacher behaviors, it is very likely that all the relations shown in Solomon and Whaler's study will appear in the naturalistic classroom observations.

\footnotetext{
${ }^{9}$ Note, for convenience and clarity, I report on the average trends across all focal children, as well as the standard deviations. These figures were computed in an Excel spreadsheet and can be made available upon request.
} 
Sage and Kindermann's (1999) study intended to capture the social contexts in which social influence over classroom behaviors occur. While their study focused on the behavior-consequence contingency, future studies can re-direct their hypotheses regarding peer and teacher differences in behavior-consequence contingency relations, towards such differences in behavior-consequence functional relations. In this way, such a study could look beyond contingency patterns and ask whether all social partners exert similar functional effects on behaviors, even when such consequences of Approval or disapproval are rare. Finally, such a study could go a step beyond that of Soloman and Whaler's by looking towards naturally occurring peer relations, and not those assigned experimentally, to assess whether certain peers (e.g., peer group members, friends, arbitrary classmates) exert more or less of an effect on subsequent behaviors, by the consequences they provide during naturally occurring classroom interactions. 


\section{Appendix B}

\section{Non-Experimental Reinforcement Detection Strategies}

The current thesis is not the first study to examine naturally occurring social reinforcement patterns. Nevertheless, it is the first study to propose a 'user-friendly' analytic software to detect consequences that alter the course of behavior probability, across time. In this way, the current thesis is unlike prior strategies in that a) it aligns with Skinner's original definition of reinforcers, b) it does not require sophisticated programming or statistical computations from user's, and c) it can be applied to any set of behavioral observations. Arguably, to build a sense of the current studies contributions to the literature, it is helpful to review prior strategies and emphasize their discrepancies with that proposed in this thesis.

The purpose of this section is to twofold: 1) draw attention to the prior strategies of tackling one goal of the current study, namely, to identify reinforcers/punishers without experimental manipulations, and 2) to distinguish these methods from the proposed strategy. I selected studies that use Skinner's original definition of reinforcers to look at consequences which lead to either long-term or short-term changes in subsequent behavioral patterns. I chose to focus on two key studies and discuss the theory/strategy/results of each study, re-direct the reader to related studies, and conclude by emphasizing the distinguishing features with the strategy proposed in this thesis.

The interested reader is re-directed to McDowell and Caron (2010) for a study which looks at global patterns of behavior and contingent consequences, as well as Strand, Whaler and Herring's (2001) study which uses a 'post-Skinnerian' theory of reinforcer magnitude as function of reinforcement history. While these methods have been used to analyze naturally occurring behavior-consequence patterns for the purpose of inferring reinforcing consequences, they have not been tested under fully naturalistic conditions, as conceptualized in this thesis (See pages 22-24), and are thus, excluded from this appendix.

\section{Reinforcement History and Long-Term Predictions}

Skinner defined reinforcers in terms of events that can change behavior probability across time. Stated generally, reinforcers can apply to developmental theories of human behavior as mechanisms that account for the development of behavior patterns. When viewed this way, a reinforcing consequence is unlike non-functional consequences, in that reinforcers can 'stamp-in' patterns of behaviors, given a stable history of reinforcement contingencies. I re-direct the interested reader to Maccoby (1992; pp.1007-1110) for an overview of studies using this notion of reinforcement history in social-developmental theory.

Patterson, Littman, \& Bricker's (1967) study is one example of a naturalistic observation which used the notion of reinforcement history to explore whether children's patterns of aggressive actions could be predicted by patterns of receiving reinforcers for prior aggressive behaviors. This study used an intraindividual analysis of learning to look for trends of change across individuals, and to link these to a common pattern of behaviors and consequences.

As a theoretically guided study, Patterson and colleagues postulated that only a certain class of consequences would have reinforcing effects, and that the cumulative frequency of these consequences would correlate with the cumulative frequency of aggressive behaviors. The following few paragraphs describe the observational and analyses strategy used to collect observations of children's social interactions and identify potential reinforcing relations between aggressive behaviors and an 'a-priori' class of social reinforcers.

Analytic Strategy and Findings. Using a sample of 21 preschoolers, Patterson and colleagues (1967) obtained 2,583 aggressive responses and their consequences, over the course of sixty, 2.5-hour continuous observation sessions, spread across a school year (from the fall to spring). In this way, longterm 2.5-hour observations were applied to sample behaviors each continuously at each session and also, to discontinuously sample sessions for the entire school year. In the absence of a baseline for the contingency, children were split into two groups; an 'acquisition' group (those with less than two assertive behaviors) and a 'maintenance' group (those with three to eight assertive behaviors), based on the output for the first 15 hours of observations. Instead of focusing on the relation ongoing changes between contingencies (i.e. contingent versus non-contingent consequences) and behaviors, these researchers measured learning in 
terms of overall output and observed contingencies, depending on one's 'baseline' level of learning, over the course of one's observed reinforcement history.

Findings. Using a visual analysis of cumulative changes in responding (and contingencies), it was shown that acquisition of aggressive initiations was a function of number of counterattacks over the course of observations. Children who were victimized (e.g. recipients of aggression) but counterattacked rarely, did not show marked gains in the initiations of aggressive acts. Counterattacking demonstrated a similar function for the students in the 'maintenance' group; these students preserved their rank order of aggressive initiations as well as aggressive counterattacks (when victimized). These findings supported the notion that one's pre-existing exposure to reinforcement contingencies, such as having a history of successful counterattacks, may lead to more unprompted forms of future behaviors, such as aggressive initiations.

Related Studies and Distinguishing Features. To my knowledge, this is the only study that tested a theory of peer-delivered reinforcers as naturally occurring developmental mechanisms under realworld conditions. By examining intra-individual patterns of behavior change, this study was able to show that aggressive responses beget aggressive behaviors, without any artificial constraints imposed by experimental methods. Nevertheless, this study relied on very demanding long-term observational protocols, which are likely to exceed the scope of most studies. In addition to its observational demands, this study required observers to manually identify behavior-response-recurrence patterns, to separate potentially reinforcing contingent consequences, from those which occur randomly.

The current thesis offers an alternative to the intense longitudinal observations and coding rules used in Patterson and colleagues' study. This applet allows users to identify any consequence that can lead to behavior change, across any stream of observations. To replicate the analyses in Patterson's study, a reinforcer analyses would need to be made within each individual participant's stream of observations. This would require the dataset to contain individual ID variables so that the user could re-format the dataset to produce a cohesive stream for each individual ID. A subsequent reinforcer scan would identify which consequences reinforce which individuals, and this information could be used to assess how common these patterns are, across all individuals sharing similar consequence patterns.

\section{The Three Term Contingency and Short-Term Predictions}

One of the core principles of behaviorism is that behavior is a function of its environments, where an environment is any stimuli external to an organism. As a stimuli which follows behaviors and shapes future behavior patterns, reinforcers are events which occur in contexts. As discussed in the Chapter 2 of this thesis, contexts can be overall environments or situations in which reinforcers occur (i.e. classrooms, math activities), or can be temporary events which occur in a sequence across time (i.e. prompts, behaviors, consequences). Skinner (1969) used the term 'contingencies of reinforcement' to describe the environmental patterns whereby reinforcement occurs. In his words:

An adequate formulation of the interaction between an organism and its environment must always specify three things (1) the occasion upon which a response occurs, (2) the response itself and (3) the reinforcing consequences. The interrelations among them are the contingencies of reinforcement (p. 7)

Snyder and Patterson (1986) used the three term contingency as an analytical device for dividing the behavior stream into 'conditions' under which aggressive behaviors occur. In this way, Patterson and Snyder looked at the antecedents and consequences of aggressive behaviors. To determine if a consequence was reinforcing, Snyder and Patterson looked for changes in behavior proportions, given that the same antecedent event occurs. Thus, this study used the antecedent-behavior sequence served as the dependent variable for these analyses. The following few paragraphs describe the statistical steps used to determine which consequences led to increases in future behavior proportions, given that the same antecedent event occurs.

Analysis strategy and findings. The data consisted of 8-hour long observation streams, for two mother child dyadic interactions in a laboratory setting. The mother-child dyads were observed across a variety of tasks (i.e. eating, playing) without any instructions or interference. The behavior streams were broken apart into antecedent-behavior sequences, and then sorted by their consequences, in a three-step analyses process described in the following paragraphs.

Antecedent-behavior-class. The first step in obtaining the dependent variable measure was to test 
mother-antecedent child behavior sequences for statistical significance, and to group these into 'action reaction classes' used as the dependent variable. By measuring all unique antecedent-behavior combinations and comparing the conditional behavior probability to the base-rate probability, each dyad produced two action reaction classes. The child was most likely agree after following maternal requests and commands and was most likely to comply after these same antecedents; thus, agreement and compliant behaviors were combined into one action reaction class for the first dyad (the second class was antecedent positive talk and behavioral positive talk). The 'action-reaction classes' for dyad two, were maternal imperative-child compliance and maternal commands-child coerce, where compliance included verbal and physical instruction following, and coerce included verbal disapproval and threats.

Controlling for extended lags. The second step was to statistically control for any extended or 'thematic' events of these 'action-reaction' classes. This step was necessary so to rule out the effects of extended interactions sharing a common theme (i.e. argument) from the effects of specific consequences outside a particular 'thematic context'. A lag sequential analysis, carried out to a maximum of 15 lags, was to identify any enduring thematic patterns in which the probability of behavior exceeded its expected base rate probability. Only the second dyad exhibited thematic effects for the class 'imperative-compliance' and 'disapprove-coerce'. The behavior probability returned to baseline after 5 lags, thus, for this dyad, behavior probability was measured for reinforcer effects only for behaviors occurring after 5 observations after one of these last observed 'action-reaction' classes. Because there were no consistent behavior-elevations, following the 'action-reaction' classes in dyad one, the reinforcer analyses was used all observations of these sequences without further modifications.

Conditional Probability Analysis. The third step was to measure changes in the conditional probability of a child's behavior given that the behavior was in an 'action reaction' class followed by either positive or negative consequences. This step entailed scanning the stream for each action reaction class and its consequence, calculating the behavior probability up to the next antecedent (from the same actionreaction class), and finally, aggregating these proportions and sorting them according to each type of consequence of the 'action-reaction' class. As a final step, the behavior proportions for each 'actionreaction' class tested for statistical significance by comparing each probability to the base rate probability in each consequence group, using a binomial z-score analysis.

Findings. In both dyads child's compliant behaviors was more likely after following positive consequences for previous compliance $(z=2.27, p<.05 ; z=3.97, p<.01)$. In dyad two, child's coercive behaviors was shown to be reinforced by positive consequences, when coercion was anteceded by a mother's disapproval $(z=3.97, p<.01)$. Additionally, coercion and compliance were both shown to be punished by the disapproval for this dyad $(z=-2.08, p<.05 ; z=-1.83, p<.1)$. These patterns speak to the short-term effects of behavior recurrence across the entire stream of observations.

Related studies and distinguishing features. Snyder and Patterson's (1986) analyses strategy offers an a-theoretic strategy for identifying potential reinforcers, simply by documenting the effects a consequence has on subsequent behavioral patterns. Unlike the strategy used in this thesis, Snyder and Patterson's method classifies a reinforcer by assessing the function of a consequence the context of a threeterm behavior sequence (i.e. antecedent-behavior-consequence). This methodology was first used to assess naturally occurring social reinforcers in Patterson's (1972) study of familial interactions, was refined by Snyder and Patterson (1986), and then subsequently used in latter quasi-experimental studies testing 'postSkinnerian' reinforcement theories in the context of mother-child interactions following behavioral interventions (e.g. Strand, Wahler, \& Herring, 2001).

Snyder and Patterson's approach differs from the proposed strategy, namely, by distinguishing behaviors according to their antecedents. Stated differently, the proposed reinforcement scan can be applied to identify consequences which alter the course of a behavior - across all of the antecedents in which behaviors occur. Using an example from this thesis, Approval for On-Task behaviors is tested as a reinforcer across all antecedent cases, that is, where On-task behavior follows passive On-task behaviors, Off-task behaviors, or any partner consequence. This contrasts with Snyder and Patterson's strategy, which would independently test the effects of social approval across each of these possible antecedent-behavior patterns.

Unlike the analyses proposed in this thesis, Snyder and Patterson's does not account for changes in the base rate recurrence of behaviors across a continuous series of observations. In this way, Snyder and Patterson's strategy re-organizes the continuous stream of sequential observations, so that target behaviors 
are organized by antecedents, and not ordered by the natural sequence of observations. Thus, this strategy offers a more precise account of events which alter the probability of behaviors within the context of shortterm sequences (i.e. antecedent - behavior), but not an account of events which change the general flow of behavior probability. 


\section{Appendix C}

\section{Glossary of Terms}

After-reinforcement sequence: A subset of events from the observation series (or sub-series) which follow a given reinforcer. See Chapter 5, pp. 37.

\section{Analysis application: See The Reinforcinator.}

Analysis selection sidebar: A side-panel in the Reinforcinator that provides the user with a variety of options for their analyses.

The recounted frequency contingency table : An $2 \times 2$ contingency table obtained by dividing the cells of the contingency table for recounted observations by the total number of sub-series.

Before-reinforcement sequence: A subset of events from the observation series (or sub-series) which occur prior to a given reinforcer. See Chapter 5, pp. 37.

Behavior-consequence contingency: A sequence in which an organism's behavior is followed by an environmental stimulus. See Chapter 2, pp. 11-12.

Center main window: A center window that displays function output from the Reinforcinator and Reformatter web-applications.

Continuous event coding: An observational sampling procedure that captures each behavior and consequence as discrete events that unfold across time (Farmer \& Schoenfeld, 1966; Lattal, Abreu \& Rodrigues, 1997). Observational datasets constructed under this procedure may have a variable length between observation 'episodes', that is, some may comprise more behaviors (and thus be longer) than others. See Chapter 2, pp. 10.

Event: I use the term events interchangeable with observations, in reference to the unit of analysis used to infer the status of a putative reinforcer. The events comprise behaviors in an observational data-file. For social interactions, events are units for the independent and dependent variables. See Chapter 5, pp. 36.

Event interval sampling: An observational sampling procedure that divides an observation interval into equally spaced intervals. Each interval contains one behavior code. See Chapter 2, pp. 10.

Focal person sampling strategy: An observational sampling strategy in which observations focus on one person's behavior at a time. See Chapter 2, pp. 11.

Long format: Long format refers to a longitudinal matrix where each row is one observation (or measurement period) and each column represents a variable to classify, or 'identify' the observations (Wickham, 2007, p. 3).

Naturalistic simulations: A term used to describe experimental studies that test reinforcement in naturalistic settings of applied interest (e.g. schools, classrooms, clinics).

Observation stream: An analogy introduced by Farmer \& Schoenfeld, 1966, and later used by Bakeman and Quera (1995) and others, for describing observations in terms of events unfolding across time. I use the term observation series exchangeabley with observation stream.

Overall index value: An event index that begins, and ends, at the first and last events in a data-file.

Overall series: A series of observations resulting from the concatenation of all observations (or events) in a given observational data-file.

Overall strategy: A term to describe a general set of detection strategies applied to all events in a given 
observation series. This strategy infers reinforcement across all secondary variables (e.g., individual ID, day of Observations). See Chapter 3, pp. 25-26.

Probabilistic contingency: A term commonly used to describe behavior-consequence contingencies that are more likely than chance (in a context of sequential lag-sequential analyses of consequence probability, conditioned on behavior antecedents). See Chapter 2, pp. 12.

Process-oriented behavior systems: A term used by Altman to describe observation systems specifically constructed for analyses of social exchange (i.e. interaction) processes. These systems contain information regarding 1) the 'actors' (i.e. source of antecedent behavior), 2) the partners (i.e. source of social consequences), 3) the sequence of events (i.e. transition between behavior and consequence), and 4) an indicator of time or duration of the events. See Chapter 2, pp. 12.

Punishers: When behaviors decrease in frequency, following a consequence, then this consequence (stimulus) is a punisher because it exhibits a reinforcing effect on behaviors. See Chapter 2, pp. 8.

Putative reinforcer: A behavior which is hypothesized (or assumed) to have reinforcing effects on naturally occurring behaviors, but without evidence demonstrating these effects, in naturalistic contexts. See Chapter 1, pp. 10.

Contingency table for recomputed frequencies: A contingency table obtained by the product of the rowconditioned proportions (for each sub-series table) and the corresponding count from the contingency table for the average recounted frequencies. See Chapter 5, pp. 45.

Recounting algorithm: An algorithm that recounts events in a given series of observations, based on each occurrence of a reinforcer. See Chapter 5, pp. 37-40.

Re-counted series: A transformation of an observation series, in which all events are re-counted based on the occurrence of reinforcers in an observation series. See Chapter 5, pp. 37-40.

The recounted data-file: A dataset resulting from the application of the overall strategy of the ReCounting algorithm to all events in an original observational data-file. See Chapter 5, pp. 39.

Reinforcer: As defined by B.F. Skinner (1953), "any consequence of behavior which is rewarding, or more technically, reinforcing, increases the probability of further responding (B. F. Skinner, 1953; p. 345)". See Chapter 2, pp. 8.

Reinforcement detection algorithm: see Re-Counting Algorithm

Reinforcement sequence: A subset of events in a series (or sub-series) which correspond to a phase of reinforcement (i.e. before or after). See Chapter 5, pp. 38.

The Reinforcinator: A set of statistical functions, presented in a graphical user interface, allowing the user to apply the recounting procedure to obtain the summary series and corresponding aggregate contingency tables. Additionally, the app provides the interface for the general estimating equation and contingency table methods described in this thesis.

Series: I use the term series to denote the sample of observations used to detect putative reinforcers. See Chapter 5, pp. 38.

Sub-series: A subset of the observation stream corresponding to a reinforcer. Each segment is of length n1 ; where $\mathrm{n}$ is the original length of observations, and the observation containing the reinforcing event is removed from the segment sample. Each segment is demarcated into two windows, one before and one after, the reinforcing event. See Chapter 5, pp. 38.

Summary series: A term I use in general reference to the Re-Counted Series and derived distributions (e.g., Average Distribution and Re-Computed Frequency Distribution). See Chapter 5, pp. 37.

Target variables/events: A specific class of behaviors that serves as either a target behavior or 
consequence in traditional analyses of social reinforcers. The current study classifies a dependent variable as either a target or a non-target event in the observation series. This classification is hypotheses dependent - a target for one hypothesis may be a non-target for a second hypothesis. 


\section{Appendix D}

\section{Glossary of Equations}

Equation 9. Binomial z test for lagged dependence

$$
z_{1}=\frac{P_{T \mid A}-P_{T}}{\sqrt{\frac{P_{T}\left(1-P_{T}\right)\left(1-P_{A}\right)}{(N-k) * P_{A}}}}
$$

This equation uses a corrected standard error for binomially distributed events separated by a lag of $k$. This equation is adapted from Allison \& Liker (1982, pp. 395).

Equation 10. Logistic link function for linear transformation of binomially distributed variables

$$
\operatorname{logit}\left(\mathrm{p}\left(\mathrm{y}=1 \mid \mathrm{x}_{i}\right)\right)=\log \frac{\mathrm{p}\left(\mathrm{y}=1 \mid \mathrm{x}_{i}\right)}{1-\mathrm{p}\left(\mathrm{y}=1 \mid \mathrm{x}_{i}\right)}
$$

This is the equation for the linear transformation of a binomially distributed variable, using the logit link function. I adapt this from Agresti (2013, pp. 163).

\section{Equation 11. Conversion of logistic coefficients to probability}

$$
\operatorname{Pr}\left(\mathrm{y}=1 \mid \mathrm{x}_{i}\right)=\frac{e^{\beta_{0}+\beta_{1} x}}{1+e^{\beta_{0}+\beta_{1} x}}
$$

This equation converts the beta coefficients from a logistic regression into conditional probability values. It is adapted from Agresti (2013, pp.163), where the outcome is denoted as $\pi(\mathrm{x})=\operatorname{Pr}(\mathrm{Y}=1 \mid \mathrm{X}=\mathrm{x})$. I adapted this equation so that $\mathrm{x}$ is a binary categorical variable, where $\mathrm{x}_{i=0}$ denotes an event in the before sequence and $\mathrm{x}_{i=1}$ denotes an event in the after-reinforcement sequence. 


\section{Appendix E}

\section{Reformatter}

This appendix reviews the 'Reformatter'. This was originally proposed as a second part of the Reinforcinator. The Reformatter was intended to allow users to combine and remove codes prior to running the recounting procedure. Time did not allow for these functions to be implemented in this thesis. These functions are intended to be incorporated into a latter revision (see Chapter 8 for the discussion).

\section{The Reformatter}

The Reformatter is a data formatting application that modifies a pre-existing observational dataset (in the form specified below) into a new file such that each row has a corresponding column value for each variable. I propose to accomplish this by providing users with options to: a) remove any unnecessary variables, $b$ ) consolidate existing variables by combining its possible values, and c) downloading the new csv file. These changes will provide the user with a dataset ready for re-structuring according to the recounting algorithm so that it contains an uninterrupted and complete series of events with the necessary information for detecting reinforcers.

The following few paragraphs begin by describing the required form for data-files, the deletion and combination functions, and ends by discussing how future revisions for the proposal or versions of the applet (given time restrictions) will allow users to change their data to appropriate form by the applet and not manually by some other software.

Pre-requisite data-file. The reformatting application accept any csv files that comes in a long format for repeated observations. Additionally, each variable must have complete observations - such that every row has a value for every column (variable). Figures 7 and 8 show the same dataset with an inappropriate versus appropriate structure. Notice that both datasets contain a variable for identifying the events that correspond to the beginning and end of a given observation series. The variable ' $\mathrm{X}$ ' in Figure 7 uses the code $\% \mathrm{~S} 1-1105 \mathrm{~A} \%$ and a semi-colon (not shown) to identify the events corresponding to series S1-1105A. Figure 8 shows an appropriate alternative for identifying the boundaries of each series. This dataset uses a 'Day.Code' variable to associate each event with a series, row-by-row.

The proposed applet requires that datasets follow the form shown in Figure 8 - and require the user to change their dataset to accommodate this structure, prior to using the formatting or analysis applications. Stated differently, the Reformatter will not transform a non-csv file into the appropriate rowby-column long structure, with complete observations. This falls outside the scope of this thesis, and users can make this conversion manually in a number of ways. If time allows, I will include a function for the user to create new variables within the applet - but otherwise reserve this feature for future versions. 
Figure 31. Example of an appropriately reformatted dataset

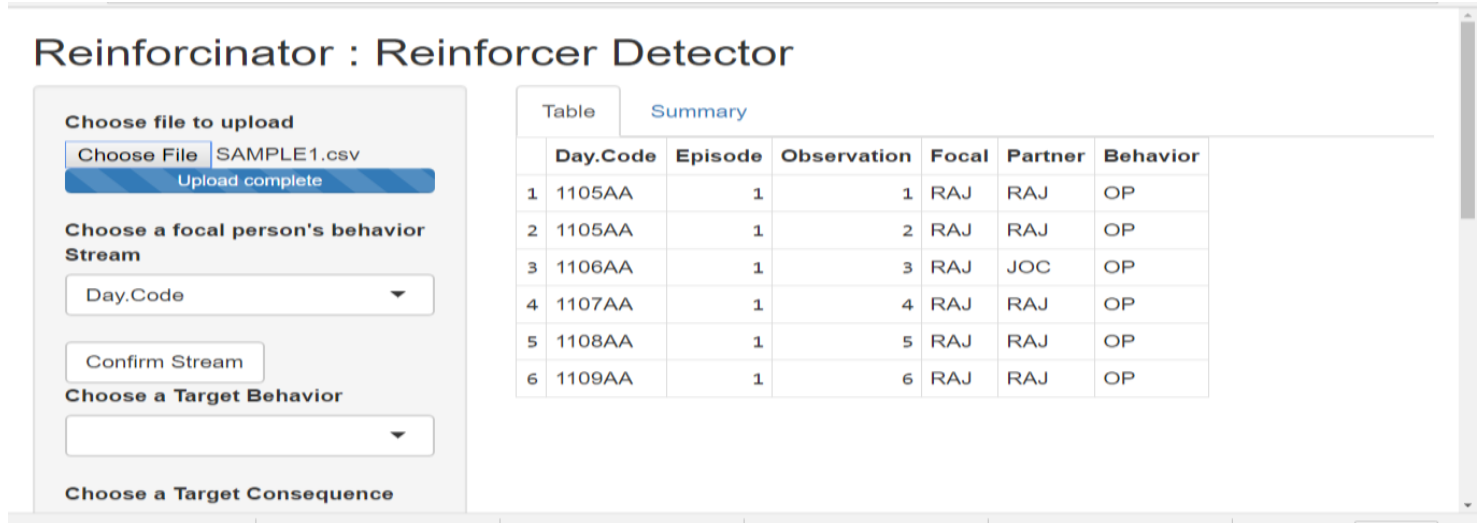

Proposed cleaning functions. I propose two primary functions to re-format the codes used in an observational data-file: 1) variable / code removal and 2) combination of event codes. The option to remove behavior codes allows a user to: a) delete specific values of a given variable, such as Cooperation codes from the event variable, or b) remove a variable entirely, such as 'description of Observations'. The user can do this for theoretical reasons, such as removing teacher interactions when looking for peer reinforcers, or perhaps for practical reasons, such as (removing unused 'end of observation' codes, or for a combination of the two, such as removing 'move out of camera' codes from the series. Any code selected for removal will alter the dataset by removing all rows (or events in an observation series) that correspond to the removed code in the original data-file.

The option to combine event codes allows users to select any cases of the event variable and combine them into one target code value. Unlike the option to remove codes, combining codes does not alter the structure of the dataset by removing rows or columns. Alternatively, combining codes only affects the measurement of event counts, and thus, effects the measures of cross-classified events and subsequent statistical analyses. This occurs by: a) increasing the proportion of target to non-target events, and / or b) increasing the number of potential reinforcers, and thus, the number of sub-intervals used to sample event proportions. The proposed feature will not place limits on the number of behaviors or consequences combined with the target variables.

Future revisions. I propose two additional features (if time allows) for the code removal/combination functions. Firstly, I wish to add the ability for users to combine codes conditionally on a second variable, such as combining day of observations given a focal child. As an alternative to combining all events with a given code, this feature would only combine or remove codes which meet some criteria (e.g., removing less than 10 observations of target events, particularly active days of observations, etc.). Secondly, I wish to add the ability to combine or remove multiple values of a variable, simultaneously. In the Sage and Kindermann (1999) set of observations, for example, this feature would allow the user to select any number of days to remove at once, or partners to combine (e.g. all peers). 


\section{Appendix F}

\section{Supplements to Analysis Strategies}

The purpose of this Appendix is to illustrate the difference between classifying observations across individuals and within individuals. Since the overall strategies classified observations independently of individuals, it could be the case that 'Jan's observations occur before a reinforcer for 'Abe's behaviors. In this case, the overall strategy would label all these as before reinforcers, even though the behavior is unrelated to the reinforcer. The GEE approach accounts for dependency within individuals, and thus, would adjust the effect estimates so to account for similar observations within indiviudals.

\section{Individual Effects in GEE}

Table 26 shows an example dataset of recounted observations with two individual focal children based on Figure 9. Each child has three observations. 'Abe' shows no change before or after reinforcers, while 'Jan' does. Notice how the overall strategy classifies behavior by reinforcement sequence across both children. Jan's behaviors, for example, are labelled as occurring in the 'after' reinforcement sequence for the first sub-series. This differs from a traditional learning perspective, which may consider her behaviors as 'before' reinforcers only if they occur on her individual observation series.

Table 27 shows a $2 \times 2$ overall contingency table for the recounted observations. As this table shows, the proportions of target behaviors are both .5 before and after reinforcers. Tables 8 break this overall analysis down across individual children and Table 28 shows the example data series. As these tables show, using the overall strategy, Abe's probability of target behaviors after reinforcers is 0 (with an individual before and after difference of -1). Jan's probability of target behavior after reinforers is .5 (with an individual before and after difference of .5). The general estimating equations use the overall probability estimates in shown Table 27, while adjusting the standard error of the estimate for the individual differences.

Figure 32. Example two-person stream (short)

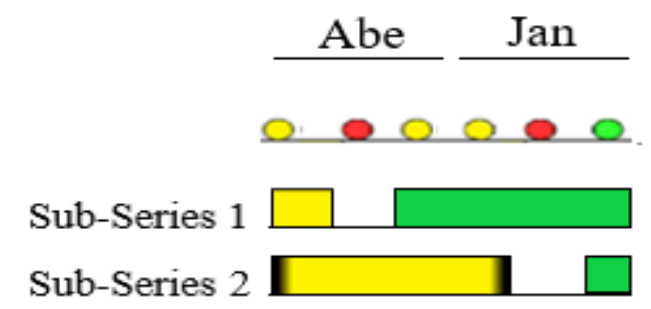


Table 25. Structure of the recounted observations for primary

\begin{tabular}{ccccc}
\multicolumn{5}{c}{ analysis } \\
\hline ID & Behavior & Recoded & Before / After & Sub Series \\
Abe & 0 & 0 & Before & 1 \\
Abe & Reinforcer & NA & NA & 1 \\
Abe & 0 & 0 & After & 1 \\
Jan & 0 & 0 & After & 1 \\
Jan & Reinforcer & 0 & After & 1 \\
Jan & 1 & 1 & After & 1 \\
Abe & 0 & 0 & Before & 2 \\
Abe & Reinforcer & 0 & Before & 2 \\
Abe & 0 & 0 & Before & 2 \\
Jan & 0 & 0 & Before & 2 \\
Jan & Reinforcer & NA & NA & 2 \\
Jan & 1 & 1 & After & 2 \\
\hline
\end{tabular}

Table 26. Contingency table for the recounted

frequencies using overall strategy

\begin{tabular}{|c|c|c|c|}
\hline & 0 & 1 & Totals \\
\hline \multicolumn{4}{|l|}{ Overall } \\
\hline Before & 5 & 0 & 5 \\
\hline After & 3 & 2 & 5 \\
\hline Totals & 8 & 2 & 10 \\
\hline \multicolumn{4}{|l|}{ Jan } \\
\hline Before & 1 & 0 & 1 \\
\hline After & 2 & 2 & 4 \\
\hline Totals & 3 & 2 & 5 \\
\hline \multicolumn{4}{|l|}{ Abe } \\
\hline Before & 5 & 0 & 1 \\
\hline After & 2 & 2 & 4 \\
\hline Totals & 3 & 2 & 5 \\
\hline
\end{tabular}


The set of all events before reinforcers follows the form of $\left(x_{i<i_{t}}^{b \in B t}\right)$, and the set of all events after reinforcers follow the form $\left(x_{i<i_{t}}^{a_{t} \in A_{t}}\right)$, where $x_{i_{t}}$ refers to the value of $\mathrm{x}$ for each location of each reinforcer $t$ in the series. For example, $i\left(x_{i_{1}}\right)=2$ (where $i\left(x_{i_{t}}\right)$ refers to the function index value of $\mathrm{x}$ ), because the first reinforcer occurs as the second event in the original series. Thus, all the pairs of events corresponding to this reinforcer follow the form $\left(x_{i<i_{t}}^{b_{1}}, x_{i>i_{t}}^{a_{1}}\right)$, and would include $\left(x_{1<i=2_{t=1}}^{1_{1}}, x_{3>i=2}^{1_{1}}\right)$, for example.

The third and final step is to assemble all the pairs of events so they can be cross-tabulated in a contingency table according to the transitions before and after reinforcers. As equation 3 indicates, this is a two step process, whereby events are first matched by a given reinforcer $t$ and then starting from the first event (i.e., $i=1$ ), each event before reinforcers is matched with an event after reinforcers. This is repeated until the end of the series, as indicated by the first union $\bigcup_{i=1}^{N}$ and repeats for each reinforcer $t$ as indicated by the second union $\bigcup_{t=1}^{M}$.

Figure 34. Overall application of recounting algorithm for classifying events by reinforcement sequence

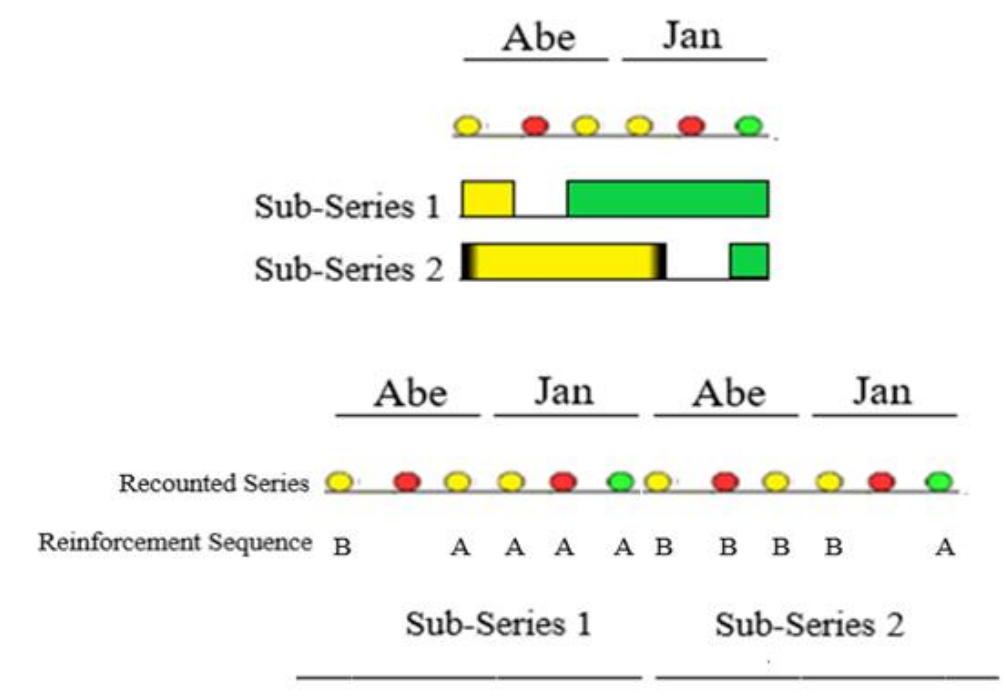

Table 27. Recounted series contingency table for two focal children

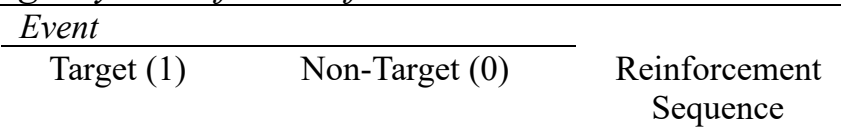


Reinforcement

Sequence

After

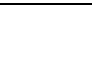

$$
3+0^{\mathrm{a}} 3+0
$$

2

Total

Before

$$
3+03+0
$$

$1+1$

$\begin{array}{lrr}\text { Before } & 5 & 0\end{array}$

$1+4$

$0+0$

5

Event Total

8

2

5

All cell values are the sum of the sub-series totals

\section{Appendix G}

\section{Detailed Review of Strategies}

Adapting the Model of Lagged Independence. The model of lagged dependence is adapted from Bakeman and Gottman (1997) and asks: What follows a specific event contingently? As discussed earlier in 
this thesis (see Chapter 5), this strategy has been used as the standard for detecting naturally occuring (putative) reinforcers. It does not detect change in a traditional behavioral sense. Alternatively, this test answers whether a specific target behavior (or reinforcing event) is more (or less) likely to follow a specified antecedent behavior (or event), than it is to occur given any other behavior (or event). Stated differently, this test compares the lag-conditioned probability of a specific behavior to all other possible combinations (at the given lag). The following few paragraphs walk through the logic of this analysis and how it can be altered to examine the variable of interest in this study, namely, to address the question of behavior change following a putative reinforcer.

Figure 35. Overall behavior stream (reprint)

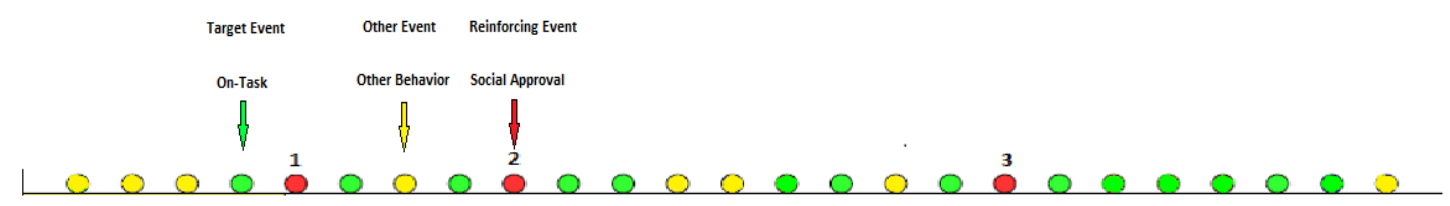

Figure 35 is used to demonstrate concepts presented in this Appendix and provide the example data for computations and tables. Table 30 shows a 2 × 2 'square' contingency table (or transition table, as it is referred to in the lag-sequential literature), for the hypothesis that red circles (consequences) follow red circles (antecedents). This table shows the counts of transitions (or pairs) of behaviors separated by lags. The term lag refers to a unit of time that separates two observations. A pair of sequentially ordered

Table 28. Lag-one conditional frequencies and proportions for red circles

\begin{tabular}{ccccc} 
& & Red & $\sim$ Red & Totals \\
\cline { 3 - 5 } Before & Red & $0(0 / 3)^{\mathrm{a}}$ & $\mathrm{F}($ Not Red | Red $)$ & \\
& $\sim$ Red & F(Red | Not Red $)$ & F(Not Red | Not Red $)$ & \\
& & $3(3 / 21)$ & $18(18 / 21)$ & 21 \\
& & & 21 & 24 \\
\hline
\end{tabular}
observations where both values are ' 1 ' would look like $\mathrm{Y}=\left(1_{\mathrm{T}}, 1_{\mathrm{T}+1}\right)$.

The lag-sequential analysis looks at whether the conditional probability of a behavior at a given time differs depending on the value of behavior one step earlier (i.e. $\operatorname{Pr}\left(\mathrm{Y}=1_{\mathrm{T}+1} \mid \mathrm{Y}=1_{\mathrm{T}}\right)($ Bakeman \& Roy, 1990, pp.17). The table can be used to test either the probability of the consequence given an antecedent or an antecedent given a consequence (as discussed in the following 'flipped' probabilities section). In either case, the cells of the transition table are obtained by isolating the sample of all antecedents and then tabulating the distribution of the immediately succeeding observations. 
Table 29. Proportions used for the Allison and Liker (1982) z-score

\begin{tabular}{lr}
\hline $\operatorname{Pr}($ Red $)$ & $3 / 25$ \\
$\operatorname{Pr}(\sim$ Red $)$ & $21 / 25$ \\
$\operatorname{Pr}($ Red $\mid \sim$ Red $)$ & $3 / 21$ \\
\hline
\end{tabular}

(Note: $\sim$ Red denotes Not Red)

The proportions shown in table 38 are used to test the hypothesis whether red (in this case) is more likely to immediately follow red (for example) than the other combinations (i.e., Red and not red, not red and red, or not red and not red). These proportions are used directly in the $z$-score for lagged observations (shown in Equation 8) corrected by Allison and Liker (1981). This equation uses the proportions shown in Table 22.

\section{Equation 10. Binomial Z test for lagged dependence}

$$
z_{1}=\frac{P(\text { red } \mid \sim r e d)-P r(\text { red })}{\sqrt{\frac{P_{\text {Red }}\left(1-\operatorname{Pr}(\text { red })\left(1-P_{\sim R e d}\right)\right.}{(N-k) * P_{R}}}}
$$

This $z$ statistic is tested using a normal approximation of the binomial distribution.

Flipped Predictions Using Sequential Analysis. The prior example shows how the transition table can answer questions about the consequence as a dependent variable. This was the type of analysis done by Sage and Kindermann (1999) to identify behavior-consequence pairs, and differences across social partners, in their study on classroom contingencies. In line with the goals of this thesis, we can flip this question to ask whether a target behavior is most likely to follow a potential reinforcer than any other consequence.

Figure 36. Observation series

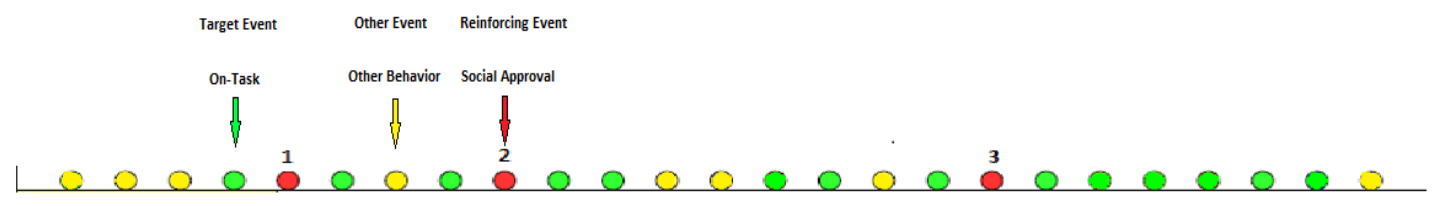

Table 32 shows the full $3 \times 3$ table based on Figure 36. With this table, we can answer questions with the dependent variable being any behavior as the antecedent or consequence. Antecedents. In line with this thesis, we may want to know whether target behaviors are more likely to follow target consequences. In this example, I use green circles for target behaviors, which are now treated as consequences for target reinforcers (shown by red circles). Table 33 shows the proportions used in the binomial $z$-score for testing whether the conditional probability of green given red is greater than chance. 
Table 30. Flipped lag-one prediction $3 \times 3$ proportions

\begin{tabular}{llccc}
\hline \multirow{3}{*}{ Before } & & \multicolumn{3}{c}{ After } \\
\cline { 3 - 5 } & Green & Green & Red & Yellow \\
\cline { 3 - 5 } & Red & 7 & 3 & 4 \\
& Yellow & 3 & 0 & 0 \\
& & 4 & 0 & 3
\end{tabular}

Table 31. Proportions used for the Allison and Liker (1982) z-score on Green Circles

$\begin{array}{rc}\operatorname{Pr}(\text { Green }) & \frac{14}{25} \\ \operatorname{Pr}(\sim \text { Green }) & \frac{3}{25} \\ \operatorname{Pr}(\text { Green } \mid \text { Red }) & \frac{3}{3}\end{array}$

Table 32. Average lag probability for green given red circles

\begin{tabular}{lcccc}
\hline & \multicolumn{3}{c}{ Reinforcer } & \\
\cline { 2 - 4 } Lag & $\mathrm{P}\left(\mathrm{G} \mid \mathrm{R}_{1}\right)$ & $\mathrm{P}\left(\mathrm{G} \mid \mathrm{R}_{2}\right)$ & $\mathrm{P}\left(\mathrm{G} \mid \mathrm{R}_{3}\right)$ & Average \\
\hline 1 & 1 & 1 & 1 & .66 \\
2 & 0 & 1 & 1 & .66 \\
3 & 1 & 0 & 1 & .33 \\
4 & 0 & 0 & 1 & 1 \\
5 & 1 & 1 & 1 & 1 \\
6 & 1 & 1 & 1 & .5 \\
7 & 0 & 0 & 0 & \\
8 & 0 & 1 & $\mathrm{NA}$ & \\
$\ldots$ & & & $\mathrm{NA}$ & \\
16 & 1 & 0 & $\mathrm{NA}$ & \\
$\ldots$ & & & & \\
20 & 0 & $\mathrm{NA}$ & & \\
Average & & & & \\
\hline
\end{tabular}


Extending the flipped predictions to pooled lags. This thesis poses the argument that a reinforcer can be detected non-experimentally, by comparing overall behavior proportions before and after the potentially reinforcing event occurs. The flipped lagged contingency is a promising strategy, namely, because it addresses behaviors as the dependent variable. Nevertheless, this does not consider the full range of observations, and thus, does not account for whether there is a change in the probability once reinforcement occurs. To account for effects across the observations, we can extend the method of lagged contingency to multiple lags and then pool (or average) the conditional proportions to look for significant conditional proportions across the entire stream of observations.

Table 33. Averaged flipped prediction proportions (Green $\mid$ Red)

\begin{tabular}{|c|c|c|c|c|}
\hline \multirow[b]{3}{*}{ Before } & & \multicolumn{3}{|c|}{ After } \\
\hline & & Green & Red & Yellow \\
\hline & $\begin{array}{l}\text { Green } \\
\text { Red } \\
\text { Yellow }\end{array}$ & $\begin{array}{l}\ldots \\
.64\end{array}$ & & \\
\hline
\end{tabular}

Table 34 shows a matrix used to capture the probability of green given red circles, across multiple lags, for each red circle. Table 35 shows how these proportions would enter a $3 \times 3$ table (using only the conditional value of green given red circles). Notice, that this strategy can effectively be seen analogously to reinforcement detection (i.e., recounting procedure) in this thesis. Unlike the recounting procedure, the strategy of extending the lags requires more computational complexity. This is because the number of pairs would be a factor of the total number of observations, which would determine the total number of lags. For datasets like Sage and Kindermann's (1999) observations, this would require over 10,000 computations for all combinations of the 6 behavior and 5 consequence codes!

Figure 37. Flipped lag analysis (both directions

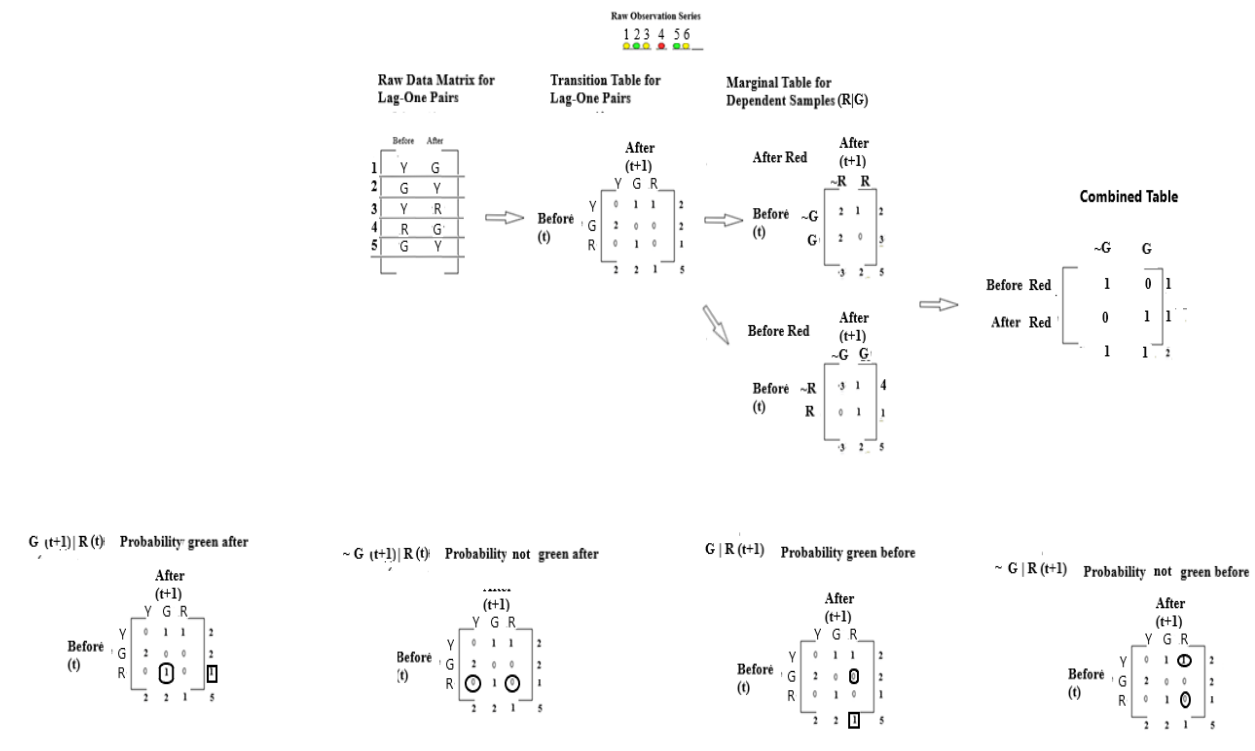

A final difference between the two strategies is that the 'flipped' lag analysis computes lags one direction at a time (i.e., testing consequence as DV or antecedent as DV). This is unlike the recounting procedure, 
which simultaneously calculates behavior in both directions and across all lags (although the contingency table strategies only compare 'after reinforcement' proportions to overall proportions. Figure 37 shows a lag-one analysis for comparing target behaviors (e.g., green circles) before and after red circles. The marginal table is then produced from two separate transition tables for collapsed categories. While this approach may not seem too demanding for a lag one analysis, the number of computations become impractical for any general purposes if this approach was extended to account for all lags. 


\section{Appendix H \\ Primary Analyses with exchangeable working correlation structure}

The purpose of this appendix is to replicate the same exact primary analyses in Chapter 7 using an exchangeable working correlation structure. This section concludes by presenting the regression summaries of each hypothesis across general estimating equations with the independence and exchangeable working correlation structures, in addition to a logistic regression that does not account for clustering.

Hypothesis 1. Hypothesis 1 states that Social Approval is a reinforcer for children's On-Task classroom behaviors. The primary test of this hypothesis compares the proportion of On-Task behaviors before and after Social Approval across all observations, under the expectation that Off-Task behaviors after reinforcers will be less than the proportion before reinforcers. A GEE model was specified with a logit link for the mean and variance of a binomially distributed outcome, and an exchangeable working correlation structure for the repeated sampling within individuals.

After adjusting for the estimated correlation within subjects $(\alpha=.101, \mathrm{SE} .=.0006)$, On-Task behaviors was estimated to be $.07 \%$ more likely to occur following Social Approval than before $\left(\beta_{1}=.035\right.$, $S E=.006$, Wald $\left.\chi^{2}(1)=273.3, p<.001\right)$. This model shows a better fit than an alternative GEE with an independence working correlation structure $\left(\mathrm{CIC}_{\text {exch }}=1.92, \mathrm{CIC}_{\text {ind }}=7.24\right)$. These findings show support for the hypothesis that Social Approval reinforces On-Task behaviors, when directly comparing the proportion of behaviors across reinforcement sequences and adjusting for the correlated response patterns within individuals.

Table 34. Research question 1 hypothesis 1 primary analysis

\begin{tabular}{cccc}
\hline Beta & $\begin{array}{c}\text { Coefficient Estimate } \\
\text { (Probability) }\end{array}$ & SE & Wald $\chi^{2}(1)$ \\
\hline$\beta_{0}$ & -1.76 & .004 & $163,961^{* * *}$ \\
$\beta_{1}$ & .035 & .006 & $73.3 .^{* * *}$ \\
\hline$* * *$ indicates $p<.001$ & & &
\end{tabular}

$* * *$ indicates $p<.001$

Hypothesis 2. Hypothesis 2 states that Social Approval is a reinforcer for children's Off-Task behaviors. The primary test of this hypothesis compares the proportion of On-Task behaviors before and after Social Approval across all observations, under the expectation that Off-Task behaviors after reinforcers will be less than the proportion before reinforcers.

A GEE model was specified with a logit link for the mean and variance of a binomially distributed outcome, and an exchangeable working correlation structure for the repeated sampling within individuals. After adjusting for the estimated correlation within subjects $(\alpha=.127, \mathrm{SE} .=.0052)$, On-Task behaviors was estimated to be $1.5 \%$ more likely to occur following Social Approval than before ( $\beta_{1}=.499, S E=.014$, Wald $\left.\chi^{2}(1)=1,288, p<.001\right)$. This model shows a better fit than an alternative GEE with an independence working correlation structure $\left(\mathrm{CIC}_{\text {exch }}=1.51, \mathrm{CIC}_{\mathrm{ind}}=8.75\right)$. These findings show support for the hypothesis that Social Approval reinforces Off-Task behaviors, when directly comparing the proportion of behaviors across reinforcement sequences and adjusting for the correlated response patterns within individuals.

Table 35. Research question 1 hypothesis 2 primary analysis

\begin{tabular}{cccc}
\hline Beta & Estimate & $S E$ & Wald $\chi^{2}(1)$ \\
\hline$\beta_{0}$ & -3.85 & .011 & $131,564^{* * *}$ \\
$\beta_{1}$ & .499 & .014 & $1288^{* * *}$ \\
\hline
\end{tabular}

a. $* * *$ indicates $p<.001$ 
Hypothesis 3. Hypothesis 3 states that Social Disapproval is a punisher for children's' Off-Task classroom behaviors. The primary test of this hypothesis compares the proportion of Off-Task behaviors before and after Social Approval across all observations, under the expectation that Off-Task behaviors after reinforcers will be less than the proportion before reinforcers. A GEE model was specified with a logit link for the mean and variance of a binomially distributed outcome, and an exchangeable working correlation structure for the repeated sampling within individuals.

After adjusting for the estimated correlation within subjects $(\alpha=.129, \mathrm{SE} .=.0118)$, Off-Task behaviors was estimated to be $1.6 \%$ more likely to occur following Social Disapproval than before $\left(\beta_{1}\right.$ $=.40, S E=.014$, Wald $\left.\chi^{2}(1)=29,399, p<.001\right)$. This model shows a better fit than an alternative GEE with an independence working correlation structure $\left(\mathrm{CIC}_{\mathrm{exch}}=1.48, \mathrm{CIC}_{\text {ind }}=8.81\right)$. These findings do not show support for the hypothesis that Social Disapproval reinforces Off-Task behaviors, when directly comparing the proportion of behaviors across reinforcement sequences, and adjusting for the correlated response patterns within individuals.

Table 36. Research question 2 hypothesis 3 primary analysis

\begin{tabular}{cccc}
\hline Beta & $\begin{array}{c}\text { Coefficient Estimate } \\
\text { (Probability) }\end{array}$ & SE. & Wald $\chi^{2}$ \\
\hline$\beta_{0}$ & -3.77 & .022 & $2,399^{* * *}$ \\
$\beta_{1}$ & .41 & .031 & $174 * * *$ \\
\hline$a . \quad * * *$ indicates $p<.001$ & &
\end{tabular}

Table 37. Comparison of GEE and logistic regression models

\begin{tabular}{cccccc}
\hline \multicolumn{5}{c}{ GEE Exchangeable } & \multicolumn{3}{c}{ GEE Independence } \\
\hline Beta & $\begin{array}{c}\text { Coefficient Estimate } \\
\text { (Probability) }\end{array}$ & SE. & $\begin{array}{c}\text { Coefficient Estimate } \\
\text { (Probability) }\end{array}$ & SE (logistic) & $\begin{array}{c}\text { SE } \\
\text { (GEE) }\end{array}$ \\
\hline $\mathrm{H} 1$ & & & & & \\
$\beta_{0}$ & $-1.76^{* * *}$ & .004 & .1 .649 & .002 & .004 \\
$\beta_{1}$ & $.035^{* * *}$ & .006 & .031 & .003 & .006 \\
$\mathrm{H} 2$ & $-3.85^{* * *}$ & .011 & -3.72 & .005 & .011 \\
$\beta_{0}$ & $.499(.038)^{* * *}$ & .014 & .525 & .007 & .014 \\
$\beta_{1}$ & & & & & .019 \\
$\mathrm{H} 3$ & $-3.77(.023)^{* * *}$ & .022 & $-3.62^{* * *}$ & .023 \\
$\beta_{0}$ & $.41(.038)^{* * *}$ & .031 & $.406^{* * *}$ & .015 & .032 \\
$\beta_{1}$ & & & & & \\
\hline
\end{tabular}

